\title{
The "Mixed" Green's Function Approach to Quantum Kinetics with Initial Correlations
}

\author{
V.G. Morozov* and G. Röpke \\ Department of Physics, Rostock University, 18051 Rostock, Germany
}

\begin{abstract}
A method for deriving quantum kinetic equations with initial correlations is developed on the basis of the nonequilibrium Green's function formalism. The method is applicable to a wide range of correlated initial states described by nonequilibrium statistical thermodynamics. Initial correlations and the real-time evolution are treated by a unified technique employing manycomponent "mixed" Green's functions. The Dyson equation for the mixed Green's function leads to a set of equations for real-time Green's functions and new (cross) components linking initial correlations with dynamical processes. These equations are used to formulate a generalized Kadanoff-Baym ansatz for correlated initial states. A non-Markovian short-time kinetic equation is derived within the $T$-matrix approximation for the self-energies. The properties of the memory kernels in this equation are considered in detail in Born approximation for the $T$-matrices. The kinetic equation is demonstrated to conserve the total energy of the system. An explicit expression for the time-dependent correlation energy is obtained.
\end{abstract}

\section{INTRODUCTION}

The problem of memory effects and initial correlations in nonequilibrium systems is as old as transport theory. In 1960s, some general aspects of this problem were studied on the basis of fundamental principles of statistical mechanics, and formally exact equations for the one-particle distribution function have been derived, involving initial correlations and memory [1] - [3]. However, since a many-particle system "forgets" irrelevant details of its initial state, the short-time memory effects and the evolution of initial correlations were long thought to be purely theoretical problems related to the justification of Boltzmannlike kinetic equations and hydrodynamic equations. Therefore, no serious attempts were made to construct explicit kinetic equations describing the early stage of evolution of a nonequilibrium system. Now this topic has come to more practical importance due to experimental studies of fast relaxation processes caused by the interaction of very short laser pulses with matter [4, 5]. Another field of high interest in short-time quantum kinetics with initial correlations are nuclear collisions [6] - 9].

*Permanent address: Department of Physics, Moscow Institute of Radioengineering, Electronics and Automation, Vernadsky Prospect 78, 117454, Moscow, Russia 
Although some interesting facts showing the interplay between collisions and correlations in a many-body system were noticed many years ago (see, e.g, [10]), a detailed investigation of short-time kinetics with initial correlations has been undertaken only recently by Kremp et al. [11, 12] using the density matrix approach. In these works, the quantum BBGKY hierarchy for the reduced density matrices is truncated at the threeparticle level. This allows one to consider the dynamics of two-particle correlations on the same footing as kinetic processes described by the one-particle distribution. Numerical calculations [12] show that if the truncation procedure is consistent with some necessary conditions, say the conservation of the total energy of the system, then the density matrix approach gives physically reasonable results for the time behavior of the Wigner function and the correlation energy. The advantage of this method is that one is dealing with the single-time reduced density matrices which are the natural ingredients of kinetic theory. On the other hand, using the truncation procedure, one has from the beginning to work with approximate equations. Then the problem arises how to justify and improve these equations in a systematic way.

It is well known that the real-time Green's function formalism, which to a large extend is based on the fundamental ideas of Kadanoff and Baym [13] and Keldysh [14], provides an alternative to the density matrix approach to kinetic theory. Within this formalism, the kinetic equation can be derived as a component of the exact Dyson equation on the Keldysh directed contour in the complex time plane. This is one of the advantages of the Green's function formalism since the structure of the kinetic equation follows directly from the Dyson equation and all approximations are only introduced for the self-energy which may be regarded as a result of partial summation of the BBGKY hierarchy. In the literature several generalizations of the Kadanoff-Baym-Keldysh formalism to arbitrary initial states are available. The most successful attempts are due to Hall [15, 16], Kukharenko and Tikhodeev [17], Danielewicz [18], Wagner [19], and Semkat et al. [20]. Physically, all these approaches are equivalent, but they differ in the description of initial states and in mathematical formulation. Up to now the main goal of such theories was to derive generalized Kadanoff-Baym equations or to construct a modified Keldysh diagram technique for the Green's functions in the presence of initial correlations. However, transport theory is conveniently formulated in terms of a kinetic equation for the Wigner function. The question then arises how to go over from the system of equations for the double-time Green's functions to a closed kinetic equation for the single-time Wigner function. This question arises in any version of the real-time Green's function formalism and is known as the reconstruction problem or the problem of ansatz. The task is to express the double-time correlation functions in terms of the Wigner function. Within the framework of the standard Kadanoff-Baym formalism (i.e., for non-correlated initial states), Lipavský et al. [21] were able to derive exact integral equations which allow one, in principle, to solve the reconstruction problem by iteration. Based on these equations, they also formulated a simple, but rather successful relation between the time correlation functions and the Wigner function, which is called the generalized Kadanoff-Baym (GKB) ansatz and now is extensively used in quantum kinetic theory (see, e.g. [5]). Clearly, for applications of the Green's function formalism to short-time quantum kinetics the recon- 
struction problem should be solved with taking account of initial correlations. This is, however, a difficult task since the derivation of the GKB ansatz [21] rests directly on the Dyson equation for the path-ordered Green's function, which in turn implies validity of Wick's theorem. For arbitrary initial states, Wick's theorem is not valid so that the real-time Green's function does not obey the Dyson equation. The possibility of deriving the Dyson equation for arbitrary initial states by introducing path-ordered Green's functions on an extended Keldysh contour was noted by Danielewicz [18] and then used by Wagner [19] to formulate a diagram expansion of such Green's functions. Unfortunately, this scheme has not been worked out to an extent that explicit kinetic equations can be derived.

The purpose of this paper is to develop a Green's function approach to short-time quantum kinetics, which applies to arbitrary initial states and provides the basis for the derivation of explicit kinetic equations. We shall show the theory "in action" and give some examples of such kinetic equations. The important ingredient of our approach is the extension of the GKB ansatz [21] to correlated initial states.

The paper is outlined as follows. In Sec. II, we touch briefly on the description of initial correlated states and discuss a link between this problem and nonequilibrium statistical thermodynamics. We also introduce the thermodynamic Green's functions describing initial correlations.

In Sec. III, the path-ordered mixed Green's functions are defined on the deformed Keldysh contour in the $(x, t)$-plane, where the $x$-variable is associated with the "imaginary evolution" caused by initial correlations and the $t$-variable corresponds to the real-time evolution governed by the Hamiltonian. The mixed Green's functions have different components depending on the branch of the contour on which the arguments are situated. In addition to the thermodynamic Green's functions and the real-time Green's functions, the formalism involves new auxiliary objects, namely the cross Green's functions with different types of evolution. The introduction of these functions is necessary to ensure that the full mixed Green's function obeys the Dyson equation on the extended contour. In many features our scheme is similar to those considered by Danielewicz [18 and Wagner [19]. However, we do not refer directly to the diagram technique because the Feynman rules for evaluating Green's functions depend on the initial statistical ensemble describing the system. This is why we prefer to formulate the theory in an algebraic language, where the self-energies are related to many-particle Green's functions.

In Sec. IV the matrix Dyson equation for the mixed Green's function is used to derive a coupled system of equations for the real-time and cross Green's functions. In particular, we obtain generalized Kadanoff-Baym equations including contributions from initial correlations. These equations are shown to be equivalent to analogous equations derived previously by diagram expansions 18 .

Section $\mathrm{V}$ is concerned with the problem of reconstruction of two-time correlation functions from the Wigner function. In the case under consideration, this problem is closely allied to the evolution of initial correlations. Starting from the Dyson equation for the mixed Green's function, we derive a set of exact integral equations providing a generalization of the reconstruction procedure developed by Lipavský et al. [21. 
In Sec. VI we consider the $T$-matrix approximation for the mixed Green's functions and obtain explicit expressions for the components of the self-energy in terms of the $T$ matrices. An essential point is that there exist exact relations between the cross components of the $T$-matrix and its real-time components. These relations are used to eliminate the cross components and derive a generalized optical theorem including the contribution from initial correlations.

Then, in Sec. VII we derive for the one-particle distribution function a short-time kinetic equation in the $T$-matrix approximation. The collision integral shows retardation and contains extra terms due to initial correlations. As a special case, the properties of the collision integral and the spectral function are investigated in Born approximation for the real-time $T$-matrices. We show that the kinetic equation conserves the total energy and find an expression for the time-dependent correlation energy. Our results are compared with results obtained within the framework of the density matrix method.

\section{DESCRIPTION OF CORRELATED INITIAL STATES}

To put our analysis into more straightforward language, we shall consider a nonrelativistic quantum system with the Hamiltonian (we are using units in which $\hbar=1$ )

$$
\begin{aligned}
\hat{H}=\hat{H}^{0} & +\hat{H}^{\prime}=\frac{1}{2 m} \int d r_{1} \nabla_{1} \psi^{\dagger}\left(r_{1}\right) \cdot \nabla_{1} \psi\left(r_{1}\right) \\
& +\frac{1}{2} \int d r_{1} d r_{2} d r_{1}^{\prime} d r_{2}^{\prime}\left\langle r_{1}^{\prime} r_{2}^{\prime}|V| r_{1} r_{2}\right\rangle \psi^{\dagger}\left(r_{2}^{\prime}\right) \psi^{\dagger}\left(r_{1}^{\prime}\right) \psi\left(r_{1}\right) \psi\left(r_{2}\right),
\end{aligned}
$$

where $r_{i}$ denotes a complete set of single-particle quantum numbers, say the position vector $\mathbf{r}_{i}$ and the spin variable $\sigma_{i}$. Integration over $r$ implies summation over discrete quantum numbers. The field operators satisfy the usual commutation relations

$$
\psi(r) \psi^{\dagger}\left(r^{\prime}\right)-\eta \psi^{\dagger}\left(r^{\prime}\right) \psi(r)=\delta\left(r-r^{\prime}\right), \quad \psi(r) \psi\left(r^{\prime}\right)-\eta \psi\left(r^{\prime}\right) \psi(r)=0,
$$

where the parameter $\eta$ is equal to -1 for fermions and +1 for bosons. From here on the delta function $\delta\left(r-r^{\prime}\right)$ includes the Kronecker delta with respect to discrete quantum numbers. If, for instance, $r=(\mathbf{r}, \sigma)$, then $\delta\left(r-r^{\prime}\right)=\delta\left(\mathbf{r}-\mathbf{r}^{\prime}\right) \delta_{\sigma \sigma^{\prime}}$.

The second term in the Hamiltonian (2.1) describes pairwise interactions between the particles. For simplicity, we shall assume that the potential $v\left(\left|\mathbf{r}_{1}-\mathbf{r}_{2}\right|\right)$ does not depend on spin. In that case the interaction amplitude has the form

$$
\left\langle r_{1}^{\prime} r_{2}^{\prime}|V| r_{1} r_{2}\right\rangle=v\left(\left|\mathbf{r}_{1}-\mathbf{r}_{2}\right|\right) \delta\left(r_{1}-r_{1}^{\prime}\right) \delta\left(r_{2}-r_{2}^{\prime}\right) \text {. }
$$

In general, the Hamiltonian contains additional terms describing interaction of the system with external fields. Taking the Hamiltonian in the form (2.1), we thus restrict ourselves to situations where the system is prepared in some initial (nonequilibrium) state at time $t_{0}$ and we are interested in the evolution of the system at times $t>t_{0}$. However, the 
theory can easily be generalized to the case where the system is subjected into a strong alternating external field.

In formulating the short-time kinetics, the first thing one must do is to specify the initial state of the system. This can be done most directly by a full many-particle statistical operator $\varrho\left(t_{0}\right)$, where $t_{0}$ is an initial time. Another possible way to describe the initial state is by the reduced $n$-particle density matrices $\varrho_{12 \cdots n}\left(t_{0}\right)$. In the $r$-representation they are defined as

$$
\left\langle r_{1} \cdots r_{n}\left|\varrho_{12 \cdots n}\left(t_{0}\right)\right| r_{1}^{\prime} \cdots r_{n}^{\prime}\right\rangle=\operatorname{Tr}\left\{\psi^{\dagger}\left(r_{n}^{\prime}\right) \cdots \psi^{\dagger}\left(r_{1}^{\prime}\right) \psi\left(r_{1}\right) \cdots \psi\left(r_{n}\right) \varrho\left(t_{0}\right)\right\} .
$$

Mathematically, these descriptions are equivalent if all reduced density matrices are given and both of them are used in the Green's function formalism with initial correlations. It is interesting to note that, in their pioneering work [13], Kadanoff and Baym start with the correlated state described by the equilibrium statistical operator

$$
\varrho\left(t_{0}\right)=\varrho_{\mathrm{eq}}=\mathrm{e}^{-\beta(\hat{H}-\mu \hat{N})} / \operatorname{Tr} \mathrm{e}^{-\beta(\hat{H}-\mu \hat{N})},
$$

where $\beta=1 / T$ is the inverse temperature and $\mu$ is the chemical potential. Here the correlation effects are incorporated through the interaction term in the Hamiltonian $\hat{H}$. However, Kadanoff and Baym did not consider the evolution of correlations since their task was quite opposite. To eliminate the influence of initial correlations, they take the limit $t_{0} \rightarrow-\infty$ and assume that the nonequilibrium evolution of the system is caused by some auxiliary external field acting on the particles. Further the most part of works devoted to quantum dynamics with initial correlations, except for the works of Danielewicz [18 and Wagner [19], was based on the description of the initial state in terms of reduced density matrices.

In this paper, we will assume that the initial statistical ensemble is specified by the corresponding statistical operator $\varrho\left(t_{0}\right)$. In thermal equilibrium $\varrho\left(t_{0}\right)$ is given by Eq. (2.5), but if the initial state deviates from thermal equilibrium, the problem of a representative statistical ensemble becomes nontrivial. The knowledge of the Hamiltonian does not suffice to determine $\varrho\left(t_{0}\right)$ and, generally speaking, one has to consider the past evolution of the system at times $t<t_{0}$. Clearly this is not the problem which we intend to study. Therefore, it is reasonable to construct the initial statistical operator using a physical information about the system at $t=t_{0}$. It is known from nonequilibrium statistical mechanics that a large class of nonequilibrium ensembles can be derived from Jaynes' maximum entropy principle [22, 23] which is a straightforward extension of Gibbs' ensemble method to nonequilibrium systems. Without going into detailed discussion of this principle and its wide use in theory of irreversible processes (see, e.g., [24, 25]), we only recapitulate here the main ideas which will be of importance in our subsequent consideration.

Let us assume that the state of a many-body system at $t=t_{0}$ is characterized by a set of observable quantities (state parameters) which are the mean values $\left\langle\hat{P}_{m}\right\rangle^{t_{0}}$ of some dynamical variables $\hat{P}_{m}$. Following Jaynes' principle, the corresponding statistical operator, usually referred to as the relevant statistical operator, is found from a maximum 
of the entropy $S=-\operatorname{Tr}\left\{\varrho^{\prime} \ln \varrho^{\prime}\right\}$ for given $\left\langle\hat{P}_{m}\right\rangle^{t_{0}}=\operatorname{Tr}\left\{\varrho^{\prime} \hat{P}_{m}\right\}$, where $\varrho^{\prime}$ is a trial statistical operator. ¿From the extremum condition $\delta S=0$ one immediately finds that the relevant statistical operator has the form

$$
\varrho\left(t_{0}\right)=\mathrm{e}^{-\hat{S}} / \operatorname{Tr}\left\{\mathrm{e}^{-\hat{S}}\right\}
$$

with

$$
\hat{S}=\sum_{m} \lambda_{m} \hat{P}_{m}
$$

The Lagrange multipliers $\lambda_{m}$ are to be evaluated from the self-consistency conditions

$$
\left\langle\hat{P}_{m}\right\rangle^{t_{0}}=\operatorname{Tr}\left\{\varrho\left(t_{0}\right) \hat{P}_{m}\right\}
$$

for given $\left\langle\hat{P}_{m}\right\rangle^{t_{0}}$. These conditions play the role of nonequilibrium equations of state. The operator $\hat{S}$ given by Eq. (2.7) may be called the entropy operator because its expectation value determines the entropy of the initial ensemble [24]. Clearly, the equilibrium distributions are a special case of the relevant statistical operators where the $\hat{P}_{m}$ correspond to additive integrals of motion. Note also that the definition of the nonequilibrium entropy as the entropy in the relevant ensemble leads to a natural extension of thermodynamic relations to nonequilibrium states characterized by macroscopic fluxes, partial equilibrium in subsystems, a nonuniform distribution of local thermodynamic quantities, etc. [24]. A more detailed description of many-particle correlations is achieved by the use of operators $\hat{P}_{m}$ corresponding to reduced density matrices $\varrho_{12 \cdots n}$. Here we will not go into a discussion of the physics contained in the description of many-particle correlations in terms of statistical operators (2.6) (see, e.g. 24, 25]). The only fact necessary for our purposes is that each of the relevant dynamical variables $\hat{P}_{m}$ in the entropy operator (2.7) can always be written as a cluster decomposition in terms of the field operators. Thus, the general form of the entropy operator is

$$
\hat{S}=\sum_{k \geq 1} \frac{1}{k !} \int d r_{1} \cdots d r_{k}^{\prime} \lambda_{k}\left(r_{1}^{\prime} \ldots r_{k}^{\prime}, r_{1} \ldots r_{k}\right) \psi^{\dagger}\left(r_{k}^{\prime}\right) \cdots \psi^{\dagger}\left(r_{1}^{\prime}\right) \psi\left(r_{1}\right) \cdots \psi\left(r_{k}\right) .
$$

The quantities $\lambda_{k}\left(r_{1}^{\prime} \ldots r_{k}^{\prime}, r_{1} \ldots r_{k}\right)$ are some functions of the Lagrange multipliers in Eq. (2.7). To find these functions, one has to specify the relevant dynamical variables $\hat{P}_{m}$. In writing Eq. (2.9), we have assumed that the initial statistical operator commutes with a particle number operator. For superfluids and superconductors, however, particle number non-conserving terms must be included [26].

The entropy operator (2.9) can be represented as a sum $\hat{S}=\hat{S}^{0}+\hat{S}^{\prime}$, where $\hat{S}^{0}$ is bilinear in the field operators $(k=1)$, and $\hat{S}^{\prime}$ collects all higher-order terms. In what follows, we shall illustrate the general theory assuming the one-particle density matrix $\varrho_{1}\left(t_{0}\right)$ and the two-particle density matrix $\varrho_{12}\left(t_{0}\right)$ to be independent state parameters. In that case the entropy operator has the form

$$
\begin{aligned}
\hat{S}=\hat{S}^{0} & +\hat{S}^{\prime}=\int d r_{1} d r_{1}^{\prime} \lambda_{1}\left(r_{1}^{\prime}, r_{1}\right) \psi^{\dagger}\left(r_{1}^{\prime}\right) \psi\left(r_{1}\right) \\
& +\frac{1}{2} \int d r_{1} d r_{2} d r_{1}^{\prime} d r_{2}^{\prime} \lambda_{2}\left(r_{1}^{\prime} r_{2}^{\prime}, r_{1} r_{2}\right) \psi^{\dagger}\left(r_{2}^{\prime}\right) \psi^{\dagger}\left(r_{1}^{\prime}\right) \psi\left(r_{1}\right) \psi\left(r_{2}\right)
\end{aligned}
$$


where the functions $\lambda_{1}$ and $\lambda_{2}$ are determined by the equations of state

$$
\begin{aligned}
& \left\langle r_{\mid} \varrho_{1}\left(t_{0}\right) \mid r_{1}^{\prime}\right\rangle=\operatorname{Tr}\left\{\psi^{\dagger}\left(r_{1}^{\prime}\right) \psi\left(r_{1}\right) \varrho\left(t_{0}\right)\right\}, \\
& \left\langle r_{1} r_{2}\left|\varrho_{12}\left(t_{0}\right)\right| r_{1}^{\prime} r_{2}^{\prime}\right\rangle=\operatorname{Tr}\left\{\psi^{\dagger}\left(r_{2}^{\prime}\right) \psi^{\dagger}\left(r_{1}^{\prime}\right) \psi\left(r_{1}\right) \psi\left(r_{2}\right) \varrho\left(t_{0}\right)\right\} .
\end{aligned}
$$

Generalization to other entropy operators would be quite straightforward.

For the relevant ensembles, it is possible to develop a Green's function formalism which provides a way of calculating many-particle averages and solving nonequilibrium equations of state by means of a diagram technique [25. To summarize some basic relations and definitions (see also Appendix A), let us introduce the "evolution operator"

$$
\mathcal{U}\left(x, x^{\prime}\right)=\mathrm{e}^{-\left(x-x^{\prime}\right) \hat{S}},
$$

where $x, x^{\prime}$ are real variables, and define dynamical variables $\hat{A}$ in the "Heisenberg picture"

$$
\hat{A}_{H}(x)=\mathcal{U}(0, x) \hat{A} \mathcal{U}(x, 0)=\mathrm{e}^{x \hat{S}} \hat{A} \mathrm{e}^{-x \hat{S}} .
$$

The one-particle and the $n$-particle thermodynamic Green's functions are then defined as [from here on the symbol $\langle\ldots\rangle$ means averaging with the initial statistical operator (2.6)]

$$
\begin{aligned}
& \mathcal{G}\left(1,1^{\prime}\right)=-\left\langle T_{x}^{c}\left(\psi_{H}(1) \psi_{H}^{\dagger}\left(1^{\prime}\right)\right)\right\rangle, \\
& \mathcal{G}^{(n)}\left(1 \cdots n, 1^{\prime} \cdots n^{\prime}\right)=(-1)^{s}\left\langle T_{x}^{c}\left(\psi_{H}(1) \cdots \psi_{H}(n) \psi_{H}^{\dagger}\left(n^{\prime}\right) \cdots \psi_{H}^{\dagger}\left(1^{\prime}\right)\right)\right\rangle,
\end{aligned}
$$

where the labels $(k)$ and $\left(k^{\prime}\right)$ indicate respectively $\left(r_{k}, x_{k}\right)$ and $\left(r_{k}^{\prime}, x_{k}^{\prime}\right)$. $T_{x}^{c}$ is the "chronological" ordering operator with respect to the values of $x$; for Fermi systems, $T_{x}^{c}$ has the usual sign convention for permutations of the field operators. In the special case of thermal equilibrium, the entropy operator is $\hat{S}=\beta(\hat{H}-\mu \hat{N})$, so that the thermodynamic Green's functions (2.14) and (2.15) go over to the well-known Matsubara-Green's functions [27], if one introduces a new variable $\tau=\beta x$ corresponding in a sense to the imaginary-time evolution.

The one-particle thermodynamic Green's function $\mathcal{G}\left(1,1^{\prime}\right)$ is a function of the difference $x_{1}-x_{1}^{\prime}$ and satisfies the Dyson equation (see Appendix A)

$$
\int_{x_{0}}^{x_{0}+1} d 1^{\prime \prime}\left\{\mathcal{G}_{0}^{-1}\left(1,1^{\prime \prime}\right)-\mathcal{K}\left(1,1^{\prime \prime}\right)\right\} \mathcal{G}\left(1^{\prime \prime}, 1^{\prime}\right)=\delta\left(1-1^{\prime}\right)
$$

where

$$
\mathcal{G}_{0}^{-1}\left(1,1^{\prime}\right)=-\left[\delta\left(r_{1}-r_{1}^{\prime}\right) \frac{\partial}{\partial x_{1}}+\lambda_{1}\left(r_{1}, r_{1}^{\prime}\right)\right] \delta\left(x_{1}-x_{1}^{\prime}\right)
$$

is the generator of "free evolution", and $\mathcal{K}\left(1,1^{\prime}\right)$ is the thermodynamic self-energy. The value of $x_{0}$ may be prescribed arbitrarily. A special choice of this parameter will be discussed below. Equation (2.16) and its adjoint can be written in the matrix form

$$
\left(\mathcal{G}_{0}^{-1}-\mathcal{K}\right) \mathcal{G}=I, \quad \mathcal{G}\left(\mathcal{G}_{0}^{-1}-\mathcal{K}\right)=I
$$


where the multiplications are defined as matrix multiplication with respect to singleparticle quantum numbers plus integration over $x$ in the interval from $x=x_{0}$ to $x=x_{0}+1$. The identity matrix is $I\left(1,1^{\prime}\right)=\delta\left(1-1^{\prime}\right)$.

\section{The “MiXeD” Green's FunCtions}

In kinetic theory, the thermodynamic Green's functions are auxiliary quantities which can be used for evaluating characteristics of initial correlations. To study the evolution of the system, one has to consider real-time Green's functions. Within the standard formalism [18, 28], the one-particle real-time Green's functions of interest can be put into a matrix Green's function defined on the Keldysh contour $C$ (see Fig. 1):

$$
G\left(1,1^{\prime}\right)=\left(\begin{array}{ll}
G^{++}\left(1,1^{\prime}\right) & G^{+-}\left(1,1^{\prime}\right) \\
G^{-+}\left(1,1^{\prime}\right) & G^{--}\left(1,1^{\prime}\right)
\end{array}\right)=\left(\begin{array}{ll}
g^{c}\left(1,1^{\prime}\right) & g^{<}\left(1,1^{\prime}\right) \\
g^{>}\left(1,1^{\prime}\right) & g^{a}\left(1,1^{\prime}\right)
\end{array}\right)
$$

where $(1)=\left(r_{1}, t_{1}\right),\left(1^{\prime}\right)=\left(r_{1}^{\prime}, t_{1}^{\prime}\right)$, and the field operators are taken in the usual Heisenberg representation with the Hamiltonian $\hat{H}$. The components of $G\left(1,1^{\prime}\right)$ are the causal Green's function $g^{c}\left(1,1^{\prime}\right)$, the anti-causal Green's function $g^{a}\left(1,1^{\prime}\right)$, and the correlation functions $g^{\gtrless}\left(1,1^{\prime}\right)$, which are defined as

$$
\begin{gathered}
g^{c / a}\left(1,1^{\prime}\right)=-i\left\langle T_{t}^{c / a}\left(\psi_{H}(1) \psi_{H}^{\dagger}\left(1^{\prime}\right)\right)\right\rangle, \\
g^{>}\left(1,1^{\prime}\right)=-i\left\langle\psi_{H}(1) \psi_{H}^{\dagger}\left(1^{\prime}\right)\right\rangle, \quad g^{<}\left(1,1^{\prime}\right)=-i \eta\left\langle\psi_{H}^{\dagger}\left(1^{\prime}\right) \psi_{H}(1)\right\rangle,
\end{gathered}
$$

where $T_{t}^{c}\left(T_{t}^{a}\right)$ is the chronological (anti-chronological) time ordering operator and the averages are calculated with the initial statistical operator $\varrho\left(t_{0}\right)$. The matrix Green's function (3.1) can also be written in a compact form

$$
G\left(1,1^{\prime}\right)=-i\left\langle T_{C}\left(\psi_{H}(1) \psi_{H}^{\dagger}\left(1^{\prime}\right)\right)\right\rangle,
$$

where $T_{C}$ is the path-ordering operator on the Keldysh contour $C$. The real-time Green's functions have the symmetry properties

$$
\left[g^{c / a}\left(1,1^{\prime}\right)\right]^{*}=-g^{a / c}\left(1^{\prime}, 1\right), \quad\left[g^{\gtrless}\left(1,1^{\prime}\right)\right]^{*}=-g^{\gtrless}\left(1^{\prime}, 1\right),
$$

which are valid for any initial statistical operator $\varrho\left(t_{0}\right)$.

The diagonal parts (with respect to time) of the correlation functions $g^{\gtrless}$ are of special importance in kinetic theory due to relations

$$
\left.i \eta g^{<}\left(1,1^{\prime}\right)\right|_{t_{1}=t_{1}^{\prime}}=\left\langle r_{1}\left|\varrho_{1}^{<}\left(t_{1}\right)\right| r^{\prime}\right\rangle,\left.\quad i g^{>}\left(1,1^{\prime}\right)\right|_{t_{1}=t_{1}^{\prime}}=\left\langle r_{1}\left|\varrho_{1}^{>}\left(t_{1}\right)\right| r^{\prime}\right\rangle,
$$

where

$$
\begin{aligned}
& \left\langle r_{1}\left|\varrho_{1}^{<}(t)\right| r^{\prime}\right\rangle \equiv\left\langle r_{1}\left|\varrho_{1}(t)\right| r^{\prime}\right\rangle=\left\langle\psi_{H}^{\dagger}\left(r_{1}^{\prime} t\right) \psi_{H}\left(r_{1} t\right)\right\rangle \\
& \left\langle r_{1}\left|\varrho_{1}^{>}\left(t_{1}\right)\right| r^{\prime}\right\rangle=\delta\left(r_{1}-r_{1}^{\prime}\right)+\eta \varrho_{1}\left(r_{1}, r_{1}^{\prime} ; t\right)
\end{aligned}
$$


are the nonequilibrium one-particle density matrices in the $r$-representation.

The crucial point in the standard real-time Green's function formalism is that, for a non-correlated initial state, the function (3.4) obeys the Dyson equation on the contour $C$. Although in most applications the existence of the Dyson equation is not considered at all, this is a nontrivial fact because it implies that the Green's function $G\left(1,1^{\prime}\right)$ has the unique inverse function $G^{-1}\left(1,1^{\prime}\right)$ on $C$. The original derivation of the Dyson equation for the nonequilibrium real-time Green's functions was based on a perturbation expansion in the interaction picture and Wick's theorem [14, 29]. More recently it was pointed out by Kremp et al. 30 that, in order to justify the existence of the Dyson equation for $G\left(1,1^{\prime}\right)$, one has to specify the initial (or boundary) condition for the two-particle Green's function

$$
G^{(2)}\left(12,1^{\prime} 2^{\prime}\right)=(-i)^{2}\left\langle T_{C}\left(\psi_{H}(1) \psi_{H}(2) \psi_{H}^{\dagger}\left(2^{\prime}\right) \psi_{H}^{\dagger}\left(1^{\prime}\right)\right)\right\rangle .
$$

For instance, it may be the condition of weakening of initial correlations in a distant past [30]:

$$
\left.\lim _{t_{1} \rightarrow-\infty} G\left(12,1^{\prime} 2^{\prime}\right)\right|_{\substack{t_{1}=t_{2} \\ t_{1}^{\prime}=t_{2}^{\prime}=t_{1}^{+}}}=G\left(1,1^{\prime}\right) G\left(2,2^{\prime}\right)+\eta G\left(1,2^{\prime}\right) G\left(2,1^{\prime}\right) .
$$

Clearly this boundary condition implies that the Keldysh contour is deformed such as $t_{0} \rightarrow$ $-\infty$ and the whole past evolution of the system must be involved, except when at $t=t_{0}$ the initial state of the system is non-correlated and, hence, the boundary condition (3.10) may be replaced by the analogous initial condition of complete weakening of correlations. Since, in general, the evolution starts from some correlated state, the statistical operator $\varrho\left(t_{0}\right)$ does not admit Wick's decomposition, so that the existence of a Dyson equation for $G\left(1,1^{\prime}\right)$ cannot be justified by a perturbative expansion in $\hat{H}^{\prime}$. Nevertheless, using the cluster decomposition of initial correlations in terms of the reduced density matrices $\varrho_{12 \cdots n}\left(t_{0}\right)$, it is possible to develop a modified diagram technique for the matrix realtime Green's function and study some general properties of equations of motion for its components [15] - 18]. We will follow, however, the idea advocated by Danielewicz [18] (see also Wagner's work [19]). We introduce a matrix Green's function $\underline{G}$ on the extended contour $\underline{C}$ from Fig. 2 involving the real-time evolution and the "imaginary evolution" governed by the entropy operator $\hat{S}$ as in the case of the thermodynamic Green's functions. The structure of $\underline{G}$ is more complicated than that of the real-time Green's function $G$ defined on the Keldysh contour. Nevertheless, by going to the "interaction picture" on the extended contour, it is possible to obtain for $\underline{G}$ an expression which is quite analogous to the expression in the standard real-time formalism with averaging over a non-correlated statistical ensemble (see Appendix B). Then, upon applying Wick's theorem to every term in the series expansion of the Green's function $\underline{G}$ in $\hat{H}^{\prime}$ and $\hat{S}^{\prime}$, the Dyson equation can be derived. In Appendix $B$ we show that the above procedure can be performed on a contour $\underline{C}$ (Fig. 2) with an arbitrary value of the parameter $x_{0}$. We will use this fact to simplify the formalism by setting $x_{0}=0$, which leads to the contour displayed in Fig. 3 . This choice has the advantage that now $\underline{G}$ has the minimal number of components. In what follows the extended contour $\underline{C}$ will always be assumed to have the form shown in Fig. 3. 
Let us start with some definitions. First of all, we introduce the variable $\xi=(t, x)$ that specifies a point on the contour $\underline{C}$ from Fig. 3 and define integrals along the contour by the rule

$$
\int_{\underline{C}} d \xi \underline{F}(\xi)=\left.\int_{t_{0}}^{\infty} d t F(t, 0)\right|_{\text {on } C^{+}}-\left.\int_{t_{0}}^{\infty} d t F(t, 0)\right|_{\text {on } C^{-}}+\left.\int_{0}^{1} d x F\left(t_{0}, x\right)\right|_{\text {on } C_{x}} .
$$

The first two integrals correspond to the chronological $\left(C^{+}\right)$and the anti-chronological $\left(C^{-}\right)$branch of the Keldysh contour $C$, respectively. To shorten notation, we shall use the underlining of functions and operators defined on the contour $\underline{C}$. The labels $(k)$ in such functions and operators mean $(k)=\left(r_{k}, \xi_{k}\right)$. It is convenient to introduce the function $\underline{\delta}(1,2)$ which plays the role of the delta function on the contour $\underline{C}$, i.e.,

$$
\int_{\underline{C}} d 1^{\prime} \underline{\delta}\left(1,1^{\prime}\right) \underline{F}\left(1^{\prime}\right)=\underline{F}(1) .
$$

According to the integration rule (3.11), we find that

$$
\underline{\delta}\left(1,1^{\prime}\right)= \begin{cases}\delta_{C}\left(1,1^{\prime}\right)=\delta\left(r_{1}-r_{1}^{\prime}\right) \delta_{C}\left(t_{1}-t_{1}^{\prime}\right) & 1,2 \in C, \\ \delta\left(x_{1}-x_{2}\right) \delta\left(r_{1}-r_{2}\right) & 1,2 \in C_{x}, \\ 0 & \text { otherwise, }\end{cases}
$$

where $\delta_{C}\left(t_{1}-t_{1}^{\prime}\right)$ is the delta function on the Keldysh contour [18, 28]:

$$
\delta_{C}\left(t_{1}-t_{1}^{\prime}\right)= \begin{cases}\delta\left(t_{1}-t_{1}^{\prime}\right) & t_{1}, t_{1}^{\prime} \in C^{+}, \\ -\delta\left(t_{1}-t_{1}^{\prime}\right) & t_{1}, t_{1}^{\prime} \in C^{-}, \\ 0 & \text { otherwise. }\end{cases}
$$

We next introduce the Heisenberg picture for operators on the contour $\underline{C}$ :

$$
\underline{\hat{A}}_{H}(\xi)=\underline{U}\left(\xi_{0}, \xi\right) \hat{A} \underline{U}\left(\xi, \xi_{0}\right),
$$

$\xi_{0}=\left(t_{0}, 0\right)$ being the point at the junction of the parts $C$ and $C_{x}$ of the contour $\underline{C}$ (see Fig. 3). The operator $\underline{U}\left(\xi_{1}, \xi_{2}\right)$ is defined in such a way as to describe the real-time evolution on $C$ and the imaginary evolution on $C_{x}$ :

$$
\underline{U}\left(\xi_{1}, \xi_{2}\right)=T_{\underline{C}} \exp \left\{-i \int_{\xi_{2}}^{\xi_{1}} \underline{\hat{\mathcal{H}}}(\xi) d \xi\right\},
$$

where $T_{\underline{C}}$ is the path-ordering operator on $\underline{C}$, and the "effective Hamiltonian" is given by

$$
\hat{\mathcal{H}}(\xi)= \begin{cases}\hat{H} & \xi \in C, \\ -i \hat{S} & \xi \in C_{x} .\end{cases}
$$

With the definition (3.15) of the Heisenberg picture on the contour $\underline{C}$, we can construct the corresponding one-particle and many-particle Green's functions. We shall call these 
functions the mixed Green's functions [25], because they involve the real-time Green's functions (on the Keldysh contour $C$ ) as well as the thermodynamic Green's functions (on the contour $C_{x}$ ). The one-particle mixed Green's function is defined as

$$
\underline{G}\left(1,1^{\prime}\right)=-i\left\langle T_{\underline{C}}\left(\underline{\psi}_{H}(1) \underline{\psi}_{H}^{\dagger}\left(1^{\prime}\right)\right)\right\rangle .
$$

The factor $(-i)$ is introduced in order to have the usual definition for the real-time components [cf. Eq.(3.4)]. Similarly, the $n$-particle mixed Green's function is defined as

$$
\underline{G}^{(n)}\left(1 \cdots n, 1^{\prime} \cdots n^{\prime}\right)=(-i)^{n}\left\langle T_{\underline{C}}\left(\underline{\psi}_{H}(1) \cdots \underline{\psi}_{H}(n) \underline{\psi}_{H}^{\dagger}\left(n^{\prime}\right) \cdots \underline{\psi}_{H}^{\dagger}\left(1^{\prime}\right)\right)\right\rangle .
$$

The difference between the mixed Green's functions and the usual real-time Green's functions is conveniently illustrated by expressing the one-particle Green's function $\underline{G}$ in terms of its components. Recalling the definition of the contour $\underline{C}$ (see Fig. 3), we write

$$
\underline{G}\left(1,1^{\prime}\right)=\left\{\begin{array}{cl}
G\left(1,1^{\prime}\right) & 1,1^{\prime} \in C, \\
\mathcal{G}^{>}\left(1,1^{\prime}\right) & 1 \in C_{x}, 1^{\prime} \in C, \\
\mathcal{G}^{<}\left(1,1^{\prime}\right) & 1 \in C, 1^{\prime} \in C_{x}, \\
i \mathcal{G}\left(1,1^{\prime}\right) & 1,1^{\prime} \in C_{x},
\end{array}\right.
$$

where $G\left(1,1^{\prime}\right)$ and $\mathcal{G}\left(1,1^{\prime}\right)$ are the matrix real-time Green's function (3.4) and the thermodynamic Green's function (2.14), respectively. It is significant that we also have to consider the cross Green's functions, $\mathcal{G}^{\gtrless}\left(1,1^{\prime}\right)$, which are constructed from Heisenberg operators with different types of evolution:

$$
\begin{aligned}
& \mathcal{G}^{>}\left(1,1^{\prime}\right)=\mathcal{G}^{>}\left(r_{1} x_{1}, r_{1}^{\prime} t_{1}^{\prime}\right)=-i\left\langle\psi_{H}\left(r_{1} x_{1}\right) \psi_{H}^{\dagger}\left(r_{1}^{\prime} t_{1}^{\prime}\right)\right\rangle, \\
& \mathcal{G}^{<}\left(1,1^{\prime}\right)=\mathcal{G}^{<}\left(r_{1} t_{1}, r_{1}^{\prime} x_{1}^{\prime}\right)=-i \eta\left\langle\psi_{H}^{\dagger}\left(r_{1}^{\prime} x_{1}^{\prime}\right) \psi_{H}\left(r_{1} t_{1}\right)\right\rangle .
\end{aligned}
$$

These functions satisfy the obvious boundary conditions

$$
\begin{array}{ll}
\left.\mathcal{G}^{>}\left(1,1^{\prime}\right)\right|_{t_{1}^{\prime}=t_{0}}=\left.i \mathcal{G}\left(1,1^{\prime}\right)\right|_{x_{1}^{\prime}=0}, & \left.\mathcal{G}^{<}\left(1,1^{\prime}\right)\right|_{t_{1}=t_{0}}=\left.i \mathcal{G}\left(1,1^{\prime}\right)\right|_{x_{1}=0}, \\
\left.\mathcal{G}^{>}\left(1,1^{\prime}\right)\right|_{x_{1}=0}=\left.g^{>}\left(1,1^{\prime}\right)\right|_{t_{1}=t_{0}}, & \left.\mathcal{G}^{<}\left(1,1^{\prime}\right)\right|_{x_{1}^{\prime}=0}=\left.g^{<}\left(1,1^{\prime}\right)\right|_{t_{1}^{\prime}=t_{0}},
\end{array}
$$

which relate them to the thermodynamic and real-time Green's functions. As we shall see later, the cross Green's functions play the crucial role in the theory.

\section{DYSON EQUATION ON THE EXTENDED CONTOUR}

As already noted, the one-particle mixed Green's function (3.18) satisfies the Dyson equation on the extended contour $\underline{C}$ (Fig. 3). This equation and its adjoint can be written in the form

$$
\begin{aligned}
& \int_{\underline{C}} d 1^{\prime \prime} \underline{G}_{0}^{-1}\left(1,1^{\prime \prime}\right) \underline{G}\left(1^{\prime \prime}, 1^{\prime}\right)=\underline{\delta}\left(1,1^{\prime}\right)+\int_{\underline{C}} d 1^{\prime \prime} \underline{\Sigma}\left(1,1^{\prime \prime}\right) \underline{G}\left(1^{\prime \prime}, 1^{\prime}\right), \\
& \int_{\underline{C}} d 1^{\prime \prime} \underline{G}\left(1,1^{\prime \prime}\right) \underline{G}_{0}^{-1}\left(1^{\prime \prime}, 1^{\prime}\right)=\underline{\delta}\left(1,1^{\prime}\right)+\int_{\underline{C}} d 1^{\prime \prime} \underline{G}\left(1,1^{\prime \prime}\right) \underline{\Sigma}\left(1^{\prime \prime}, 1^{\prime}\right) .
\end{aligned}
$$


The operator $\underline{G}_{0}^{-1}$ is defined as

$$
\underline{G}_{0}^{-1}\left(1,1^{\prime}\right)=\left\{\begin{array}{cl}
G_{0}^{-1}\left(1,1^{\prime}\right) & 1,1^{\prime} \in C \\
-i \mathcal{G}_{0}^{-1}\left(1,1^{\prime}\right) & 1,1^{\prime} \in C_{x} \\
0 & \text { otherwise }
\end{array}\right.
$$

where

$$
G_{0}^{-1}\left(1,1^{\prime}\right)=\left(i \frac{\partial}{\partial t_{1}}+\frac{\nabla_{1}^{2}}{2 m}\right) \delta_{C}\left(1,1^{\prime}\right),
$$

and $\mathcal{G}_{0}^{-1}\left(1,1^{\prime}\right)$ is given by Eq. (2.17). The components of the matrix self-energy $\underline{\Sigma}\left(1,1^{\prime}\right)$ in Eqs (4.1) and (4.2) will be denoted by

$$
\underline{\Sigma}\left(1,1^{\prime}\right)=\left\{\begin{aligned}
\Sigma\left(1,1^{\prime}\right) & 1,1^{\prime} \in C, \\
\mathcal{K}^{<}\left(1,1^{\prime}\right) & 1 \in C, 1^{\prime} \in C_{x}, \\
\mathcal{K}^{>}\left(1,1^{\prime}\right) & 1 \in C_{x}, 1^{\prime} \in C, \\
-i \mathcal{K}\left(1,1^{\prime}\right) & 1,1^{\prime} \in C_{x},
\end{aligned}\right.
$$

where $\Sigma\left(1,1^{\prime}\right)$ is the matrix self-energy on the Keldysh contour $C$ [18, 28]:

$$
\Sigma=\left(\begin{array}{cc}
\Sigma^{++} & \Sigma^{+-} \\
\Sigma^{-+} & \Sigma^{--}
\end{array}\right)=\left(\begin{array}{cc}
\Sigma^{c} & \Sigma^{<} \\
\Sigma^{>} & \Sigma^{a}
\end{array}\right) .
$$

In the last line of Eq. (4.5) the factor $(-i)$ is chosen from the requirement that the thermodynamic component of the mixed Green's function satisfy Eqs. (2.18) with the self-energy $\mathcal{K}$ (see below). The Dyson equations (4.1) and (4.2) will serve as the basis for our study of short-time dynamics with correlated initial states. First we wish to discuss some general consequences of these equations, which do not depend on the explicit form of the self-energy $\underline{\Sigma}$. For this purpose, we shall consider Eqs. (4.1) and (4.2) for different components of the mixed Green's function $\underline{G}$.

\section{A. Real-time components of the mixed Green's function}

Suppose that in Eqs. (4.1) and (4.2) the arguments 1 and $1^{\prime}$ correspond to the Keldysh part $C$ of the contour $\underline{C}$. Then, since the part $C_{x}$ is later along $\underline{C}$ than $C$ (see Fig. 3), we have

$$
\begin{aligned}
&\left(i \frac{\partial}{\partial t_{1}}+\frac{\nabla_{1}^{2}}{2 m}\right) G\left(1,1^{\prime}\right)=\delta_{C}\left(1,1^{\prime}\right)+\int_{C} d 1^{\prime \prime} \Sigma\left(1,1^{\prime \prime}\right) G\left(1^{\prime \prime}, 1^{\prime}\right) \\
&+\int_{C_{x}} d 1^{\prime \prime} \mathcal{K}^{<}\left(1,1^{\prime \prime}\right) \mathcal{G}^{>}\left(1^{\prime \prime}, 1^{\prime}\right), \\
&\left(-i \frac{\partial}{\partial t_{1}^{\prime}}+\frac{\nabla_{1^{\prime}}^{2}}{2 m}\right) G\left(1,1^{\prime}\right)=\delta_{C}\left(1,1^{\prime}\right)+\int_{C} d 1^{\prime \prime} G\left(1^{\prime}, 1^{\prime \prime}\right) \Sigma\left(1^{\prime \prime}, 1^{\prime}\right) \\
&+\int_{C_{x}} d 1^{\prime \prime} \mathcal{G}^{<}\left(1,1^{\prime \prime}\right) \mathcal{K}^{>}\left(1^{\prime \prime}, 1^{\prime}\right) .
\end{aligned}
$$


These are still matrix equations because $G\left(1,1^{\prime}\right)$ and $\Sigma\left(1,1^{\prime}\right)$ have different components depending on the position of the arguments 1 and $1^{\prime}$ on the Keldysh contour $C$. Taking the $( \pm)$ - components of Eqs. (4.7) and (4.8), and then using expressions (3.1), (3.14), and (4.6) together with the integration rule

$$
\int_{C} d 1 F(1)=\int_{t_{0}}^{\infty} d 1\left\{\left.F(1)\right|_{\text {on } C^{+}}-\left.F(1)\right|_{\text {on } C^{-}}\right\}
$$

one can easily obtain a system of equations for $g^{c / a}$ and $g^{\gtrless}$. It is convenient, however, to use retarded $\left(g^{R}, \Sigma^{R}\right)$ and advanced $\left(g^{A}, \Sigma^{A}\right)$ functions instead of the causal $\left(g^{c}, \Sigma^{c}\right)$ and and anti-causal $\left(g^{a}, \Sigma^{a}\right)$ ones. The conventional definitions [18, 28] are

$$
\begin{aligned}
& g^{R}=g^{c}-g^{<}=g^{>}-g^{a}, \quad g^{A}=g^{c}-g^{>}=g^{<}-g^{a}, \\
& \Sigma^{R}=\Sigma^{c}-\Sigma^{<}=\Sigma^{>}-\Sigma^{a}, \quad \Sigma^{A}=\Sigma^{c}-\Sigma^{>}=\Sigma^{<}-\Sigma^{a} .
\end{aligned}
$$

Note also that

$$
g^{R / A}\left(1,1^{\prime}\right)= \pm \theta\left[ \pm\left(t_{1}-t_{1}^{\prime}\right)\right]\left\{g^{>}\left(1,1^{\prime}\right)-g^{<}\left(1,1^{\prime}\right)\right\} .
$$

Further manipulations with Eqs. (4.7) and (4.8) are straightforward and similar to those in the standard real-time Green's function formalism (see, e.g., [28]). The only point of importance is that, by definition, the cross functions $\mathcal{G}^{\gtrless}$ and $\mathcal{K}^{\gtrless}$ do not depend on the position of their time argument on the Keldysh contour $C$. Taking this property into account, Eq. (4.7), when written in terms of $g^{\gtrless}$ and $g^{R / A}$, becomes

$$
\begin{gathered}
\left(i \frac{\partial}{\partial t_{1}}+\frac{\nabla_{1}^{2}}{2 m}\right) g^{\gtrless}\left(1,1^{\prime}\right)=\int_{t_{0}}^{\infty} d 1^{\prime \prime}\left\{\Sigma^{R}\left(1,1^{\prime \prime}\right) g^{\gtrless}\left(1^{\prime \prime}, 1^{\prime}\right)+\Sigma^{\gtrless}\left(1,1^{\prime \prime}\right) g^{A}\left(1^{\prime \prime}, 1^{\prime}\right)\right\} \\
+\int_{C_{x}} d 1^{\prime \prime} \mathcal{K}^{<}\left(1,1^{\prime \prime}\right) \mathcal{G}^{>}\left(1^{\prime \prime}, 1^{\prime}\right), \\
\left(i \frac{\partial}{\partial t_{1}}+\frac{\nabla_{1}^{2}}{2 m}\right) g^{R / A}\left(1,1^{\prime}\right)=\delta\left(1-1^{\prime}\right)+\int_{t_{0}}^{\infty} d 1^{\prime \prime} \Sigma^{R / A}\left(1,1^{\prime \prime}\right) g^{R / A}\left(1^{\prime \prime}, 1^{\prime}\right) .
\end{gathered}
$$

The adjoint equations follow from Eq. (4.8):

$$
\begin{gathered}
\left(-i \frac{\partial}{\partial t_{1}^{\prime}}+\frac{\nabla_{1^{\prime}}^{2}}{2 m}\right) g^{\gtrless}\left(1,1^{\prime}\right)=\int_{t_{0}}^{\infty} d 1^{\prime \prime}\left\{g^{R}\left(1,1^{\prime \prime}\right) \Sigma^{\gtrless}\left(1^{\prime \prime}, 1^{\prime}\right)+g^{\gtrless}\left(1,1^{\prime \prime}\right) \Sigma^{A}\left(1^{\prime \prime}, 1^{\prime}\right)\right\} \\
+\int_{C_{x}} d 1^{\prime \prime} \mathcal{G}^{<}\left(1,1^{\prime \prime}\right) \mathcal{K}^{>}\left(1^{\prime \prime}, 1^{\prime}\right), \\
\left(-i \frac{\partial}{\partial t_{1}^{\prime}}+\frac{\nabla_{1^{\prime}}^{2}}{2 m}\right) g^{R / A}\left(1,1^{\prime}\right)=\delta\left(1-1^{\prime}\right)+\int_{t_{0}}^{\infty} d 1^{\prime \prime} g^{R / A}\left(1,1^{\prime \prime}\right) \Sigma^{R / A}\left(1^{\prime \prime}, 1^{\prime}\right) .
\end{gathered}
$$

Again it is convenient to go over to a compact matrix notation. We define for functions of $t$ (on the real-time axis) and $x$ the multiplication as integration over the intervals 
$t_{0}<t<\infty$ and $0<x<1$, respectively, plus matrix multiplication with respect to single-particle quantum numbers. Then the above equations for $g^{\gtrless}$ and $g^{R / A}$ take the form

$$
\begin{aligned}
& \left(g_{0}^{-1}-\Sigma^{R}\right) g^{\gtrless}=\Sigma^{\gtrless} g^{A}+\mathcal{K}^{<} \mathcal{G}^{>}, \\
& \left(g_{0}^{-1}-\Sigma^{R / A}\right) g^{R / A}=I, \\
& g^{\gtrless}\left(g_{0}^{-1}-\Sigma^{A}\right)=g^{R} \Sigma^{\gtrless}+\mathcal{G}^{<} \mathcal{K}^{>}, \\
& g^{R / A}\left(g_{0}^{-1}-\Sigma^{R / A}\right)=I,
\end{aligned}
$$

where $I\left(1,1^{\prime}\right)=\delta\left(1-1^{\prime}\right)$ plays the role of the identity matrix, and

$$
g_{0}^{-1}\left(1,1^{\prime}\right)=\left(i \frac{\partial}{\partial t_{1}}+\frac{\nabla_{1}^{2}}{2 m}\right) \delta\left(1-1^{\prime}\right) .
$$

Equations (4.18) and (4.20) are formally identical with equations for the retarded and advanced Green's functions in the standard real-time formalism [18, 28], while Eqs. (4.17) and (4.19), which are modified Kadanoff-Baym equations, contain explicit contributions from initial correlations. Note that the terms associated with initial correlations, $\mathcal{K}<\mathcal{G}^{>}$ and $\mathcal{G}^{<} \mathcal{K}^{>}$, enters into Eqs. (4.17) and (4.19) as source terms. On the other hand, a perturbative diagram expansion [18] leads to modified Kadanoff-Baym equations in which initial correlations appear as some corrections to the self-energies $\Sigma^{\gtrless}$. In Appendix $\mathrm{C}$ we show that, in fact, these two descriptions are equivalent.

\section{B. The thermodynamic component of the mixed Green's function}

Let us turn back to the Dyson equation (4.1) and take the arguments $1=\left(r_{1}, x_{1}\right)$ and $1^{\prime}=\left(r^{\prime}, x_{1}^{\prime}\right)$ on the part $C_{x}$ of the contour $\underline{C}$ (Fig. 3$)$. Then we arrive at the equation

$$
\int_{C_{x}} d 1^{\prime \prime}\left\{\mathcal{G}_{0}^{-1}\left(1,1^{\prime \prime}\right)-\mathcal{K}\left(1,1^{\prime \prime}\right)\right\} \mathcal{G}\left(1^{\prime \prime}, 1^{\prime}\right)=\delta\left(1-1^{\prime}\right)+\int_{C} d 1^{\prime \prime} \mathcal{K}>\left(1,1^{\prime \prime}\right) \mathcal{G}^{<}\left(1^{\prime \prime}, 1^{\prime}\right)
$$

Since the values of $\mathcal{K}^{>}\left(1,1^{\prime \prime}\right)$ and $\mathcal{G}^{>}\left(1^{\prime \prime}, 1^{\prime}\right)$ do not depend on whether the argument $1^{\prime \prime}$ corresponds to the chronological $\left(C^{+}\right)$or the anti-chronological $\left(C^{-}\right)$branch of the Keldysh contour, the last term in Eq. (4.22) is zero by virtue of the integration rule (4.9). We see that the thermodynamic component of $\underline{G}$ satisfies the Dyson equation which is not connected with the real-time evolution on the Keldysh contour $C$. The above conclusion, however, is almost trivial because $\mathcal{G}\left(1,1^{\prime}\right)$ is the thermodynamic Green's function (2.14) with the imaginary evolution governed by $\hat{S}$. Nevertheless, the point to remember is that the existence of a closed Dyson equation for $\mathcal{G}\left(1,1^{\prime}\right)$ means that it has an inverse function $\mathcal{G}^{-1}\left(1,1^{\prime}\right)$ on $C_{x}$ and that the component $\mathcal{K}$ of the self-energy (4.5) is not connected with the real-time evolution of the system. 


\section{The cross components of the mixed Green's function}

To complete our general discussion of the Dyson equation for $\underline{G}$, we now derive equations of motion for the cross components, $\mathcal{G}^{\gtrless}$. Again we turn to Eq. (4.1) and take $1 \in C$, $1^{\prime} \in C_{x}$. Then we obtain

$$
\int_{C} d 1^{\prime \prime}\left\{G_{0}^{-1}\left(1,1^{\prime \prime}\right)-\Sigma\left(1,1^{\prime \prime}\right)\right\} \mathcal{G}^{<}\left(1^{\prime \prime}, 1^{\prime}\right)=i \int_{C_{x}} d 1^{\prime \prime} \mathcal{K}^{<}\left(1,1^{\prime \prime}\right) \mathcal{G}\left(1^{\prime \prime}, 1^{\prime}\right)
$$

Here the argument $t_{1}$ may be assigned to either of the two branches of the Keldysh contour $C$. It can easily be checked that in both cases we get the same equation. In the matrix form, it reads

$$
\left(g_{0}^{-1}-\Sigma^{R}\right) \mathcal{G}^{<}=i \mathcal{K}^{<} \mathcal{G} .
$$

This equation describes the evolution of $\mathcal{G}^{<}\left(1,1^{\prime}\right)=\mathcal{G}^{<}\left(r_{1} t_{1}, r_{1}^{\prime} x_{1}^{\prime}\right)$ with respect to its realtime argument. To derive the equation describing the "imaginary" evolution of $\mathcal{G}^{<}$, we use Eq. (4.2) where we take $1 \in C$ and $1^{\prime} \in C_{x}$. This gives

$$
\int_{C_{x}} d 1^{\prime \prime} \mathcal{G}^{<}\left(1,1^{\prime \prime}\right)\left\{\mathcal{G}_{0}^{-1}\left(1^{\prime \prime}, 1^{\prime}\right)-\mathcal{K}\left(1^{\prime \prime}, 1^{\prime}\right)\right\}=i \int_{C} d 1^{\prime \prime} G\left(1,1^{\prime \prime}\right) \mathcal{K}^{<}\left(1^{\prime \prime}, 1^{\prime}\right) .
$$

Again, the time argument $t_{1}$ may be taken on either of the two branches of the Keldysh contour. Assuming for definiteness that $t_{1} \in C^{+}$, we get the equation which is written in the matrix form as

$$
\mathcal{G}^{<}\left(\mathcal{G}_{0}^{-1}-\mathcal{K}\right)=i g^{R} \mathcal{K}^{<}
$$

The analogous procedure can be repeated for $\mathcal{G}^{>}\left(1,1^{\prime}\right)=\mathcal{G}^{>}\left(r_{1} x_{1}, r_{1}^{\prime} t_{1}^{\prime}\right)$. As a result we have two equations

$$
\begin{aligned}
\mathcal{G}^{>}\left(g_{0}^{-1}-\Sigma^{A}\right) & =i \mathcal{G} \mathcal{K}^{>}, \\
\left(\mathcal{G}_{0}^{-1}-\mathcal{K}\right) \mathcal{G}^{>} & =i \mathcal{K}^{>} g^{A},
\end{aligned}
$$

which describe the real-time and imaginary evolution of $\mathcal{G}^{>}$.

\section{Relation between $\underline{\Sigma}$ and the two-particle mixed Green's function}

To obtain explicit expressions for the components of the self-energy $\underline{\Sigma}$, one has to specify the form of the Hamiltonian $\hat{H}$ and the entropy operator $\hat{S}$. If these operators are given by Eqs. (2.1) and (2.10), then the matrix self-energy $\underline{\Sigma}$ can be expressed in terms of the two-particle mixed Green's function $\underline{G}^{(2)}$ (see Appendix D):

$$
\begin{aligned}
\underline{\Sigma}\left(1,1^{\prime}\right) & =i \eta \int_{\underline{C}} d 2 d 1^{\prime \prime} d 2^{\prime \prime} d 1^{\prime \prime \prime} \underline{V}\left(12,1^{\prime \prime} 2^{\prime \prime}\right) \underline{G}^{(2)}\left(1^{\prime \prime} 2^{\prime \prime}, 1^{\prime \prime \prime} 2^{+}\right) \underline{G}^{-1}\left(1^{\prime \prime \prime}, 1^{\prime}\right) \\
& =i \eta \int_{\underline{C}} d 2 d 1^{\prime \prime} d 2^{\prime \prime} d 1^{\prime \prime \prime} \underline{G}-1\left(1,1^{\prime \prime \prime}\right) \underline{G}
\end{aligned}
$$


where the symbols $k^{+}=\left(r_{k}, \xi_{k}^{+}\right)$and $k^{-}=\left(r_{k}, \xi_{k}^{-}\right)$denote points infinitesimally later (earlier) on the contour $\underline{C}$ than $k=\left(r_{k}, \xi_{k}\right)$. The "interaction matrix" $\underline{V}$ on the contour $\underline{C}$ is defined as

$$
\underline{V}\left(12,1^{\prime} 2^{\prime}\right)= \begin{cases}V\left(12,1^{\prime} 2^{\prime}\right) & 1,2,1^{\prime}, 2^{\prime} \in C, \\ i \mathcal{V}\left(12,1^{\prime} 2^{\prime}\right) & 1,2,1^{\prime}, 2^{\prime} \in C_{x}, \\ 0 & \text { otherwise, }\end{cases}
$$

where

$$
\begin{aligned}
& V\left(12,1^{\prime} 2^{\prime}\right)=\left\langle r_{1} r_{2}|V| r_{1}^{\prime} r_{2}^{\prime}\right\rangle \delta_{C}\left(t_{1}-t_{2}\right) \delta_{C}\left(t_{1}-t_{1}^{\prime}\right) \delta_{C}\left(t_{1}^{\prime}-t_{2}^{\prime}\right), \\
& \mathcal{V}\left(12,1^{\prime} 2^{\prime}\right)=-\lambda_{2}\left(r_{1} r_{2}, r_{1}^{\prime} r_{2}^{\prime}\right) \delta\left(x_{1}-x_{2}\right) \delta\left(x_{1}-x_{1}^{\prime}\right) \delta\left(x_{1}^{\prime}-x_{2}^{\prime}\right) .
\end{aligned}
$$

Note that the matrix $\underline{V}$ has the symmetry property

$$
\underline{V}\left(12,1^{\prime} 2^{\prime}\right)=\underline{V}\left(21,2^{\prime} 1^{\prime}\right),
$$

which reflects the fact that $\hat{H}$ and $\hat{S}$ are self-adjoint operators.

\section{The Problem of ANSATZ For CORRElATED INITIAL STATES}

A precursor of kinetic equations can be obtained from the generalized Kadanoff-Baym equations (4.17) and (4.19) for $g^{<}$. Subtracting these equations, we have

$$
\left[g_{0}^{-1}, g^{<}\right]=\Sigma^{R} g^{<}-g^{<} \Sigma^{A}+\Sigma^{<} g^{A}-g^{R} \Sigma^{<}+\mathcal{K}^{<} \mathcal{G}^{>}-\mathcal{G}^{<} \mathcal{K}^{>} \text {. }
$$

The first step to a kinetic equation for the one-particle density matrix is to take the diagonal part of Eq. (5.1) with respect to time variables. Then the left-hand side can exactly be expressed in terms of $\varrho_{1}(t)$, while the right-hand side still contains double-time correlation functions $g^{\gtrless}$. In order to convert Eq. (5.1) into a closed equation for $\varrho_{1}(t)$, one has to find $g^{\gtrless}$ as functionals of the one-particle density matrix. In other words, this is the point where the problem of ansatz arises. The simple ansatz proposed by Kadanoff and Baym (KB ansatz) [13] works only in situations where the quasiparticle picture is adequate to describe the evolution of the system and memory effects can be neglected. In succeeded years efforts were directed towards refining the KB ansatz for special problems of kinetic theory. As already mentioned in Introduction, an important result along this line has been obtained by Lipavský et al. [21]. These authors have derived exact integral equations which allow, in principle, to find $g^{\gtrless}$ in terms of $\varrho_{1}$ by iteration or, at least, to find corrections to the $\mathrm{KB}$ ansatz in a self-consistent way, including memory effects and dynamical correlations. It should be noted, however, that the derivation of the generalized Kadanoff-Baym ansatz (the GKB ansatz) in Ref. [21] is based on the boundary condition of complete weakening of correlations in a distant past. Therefore we have to re-formulate the GKB ansatz for $g^{\gtrless}$ in such a way as to take initial correlations into account. 
Another new feature of the short-time kinetics is the appearance of the cross Green's functions in Eq. (5.1). There are two possible approaches to these functions. The first is to use Eqs. (4.24) and (4.27) as independent equations together with a kinetic equation for $\varrho_{1}(t)$. The second is to eliminate these functions by means of some ansatz. In this section we will derive exact equations which allow one to formulate a generalized ansatz for the cross Green's functions in just the same way as for the correlation functions $g^{\gtrless}$.

We start with the correlation functions $g^{\gtrless}$ and follow the line of reasoning which is close to that of the work of Lipavský et al. [21]. In order to preserve causality, it is convenient to introduce two auxiliary correlation functions (denoting only the time arguments)

$$
G_{R}^{\gtrless}\left(t_{1}, t_{2}\right)=\theta\left(t_{1}-t_{2}\right) g^{\gtrless}\left(t_{1}, t_{2}\right), \quad G_{A}^{\gtrless}\left(t_{1}, t_{2}\right)=\theta\left(t_{2}-t_{1}\right) g^{\gtrless}\left(t_{1}, t_{2}\right) .
$$

Then, for $t_{1} \neq t_{2}$, we have

$$
g^{\gtrless}\left(t_{1}, t_{2}\right)=G_{R}^{\gtrless}\left(t_{1}, t_{2}\right)+G_{A}^{\gtrless}\left(t_{1}, t_{2}\right) .
$$

Strictly speaking, this relation does not determine $g^{\gtrless}$ for $t_{1}=t_{2}$ because $G_{R / A}^{\gtrless}$ are discontinuous at this point. One can complete, however, the definition of $g^{\gtrless}$ at $t_{1}=t_{2}$ by the limits $t_{1} \rightarrow t_{2} \pm 0$ which give the same result. Since the representation (5.3) will always be used in integrals over time, the above refinement is irrelevant.

Let us now derive the equation of motion for $G_{R}^{\gtrless}$. Differentiation with respect to $t_{1}$ gives

$$
i \frac{\partial}{\partial t_{1}} G_{R}^{\gtrless}\left(t_{1}, t_{2}\right)=i \delta\left(t_{1}-t_{2}\right) g^{\gtrless}\left(t_{2}, t_{2}\right)+\theta\left(t_{1}-t_{2}\right) i \frac{\partial}{\partial t_{1}} g^{\gtrless}\left(t_{1}, t_{2}\right) .
$$

The time derivative in the last term can be eliminated with the help of the KadanoffBaym equation (4.13). Using also the representation (5.3) in the time integral, Eq. (5.4) becomes

$$
\begin{gathered}
\left(i \frac{\partial}{\partial t_{1}}+\frac{\nabla_{1}^{2}}{2 m}\right) G_{R}^{\gtrless}\left(t_{1}, t_{2}\right)-\int_{t_{0}}^{\infty} d t_{3} \Sigma^{R}\left(t_{1}, t_{3}\right) G_{R}^{\gtrless}\left(t_{3}, t_{2}\right)=i \delta\left(t_{1}-t_{2}\right) g^{\gtrless}\left(t_{2}, t_{2}\right) \\
+\theta\left(t_{1}-t_{2}\right) \int_{t_{0}}^{\infty} d t_{3}\left\{\Sigma^{\gtrless}\left(t_{1}, t_{3}\right) g^{A}\left(t_{3}, t_{2}\right)+\Sigma^{R}\left(t_{1}, t_{3}\right) G_{A}^{\gtrless}\left(t_{3}, t_{2}\right)\right\} \\
+\theta\left(t_{1}-t_{2}\right) \mathcal{K}^{<}\left(t_{1}\right) \mathcal{G}^{>}\left(t_{2}\right) .
\end{gathered}
$$

A formal solution of this equation satisfying the required boundary condition at $t_{1}=t_{2}$ is given by

$$
\begin{aligned}
& G_{R}^{\gtrless}\left(t_{1}, t_{2}\right)=i g^{R}\left(t_{1}, t_{2}\right) g^{\gtrless}\left(t_{2}, t_{2}\right)+\int_{t_{2}}^{t_{1}} d t_{3} g^{R}\left(t_{1}, t_{3}\right) \mathcal{K}^{<}\left(t_{3}\right) \mathcal{G}^{>}\left(t_{2}\right) \\
& \quad+\int_{t_{2}}^{t_{1}} d t_{3} \int_{t_{0}}^{\infty} d t_{4} g^{R}\left(t_{1}, t_{3}\right)\left\{\Sigma^{\gtrless}\left(t_{3}, t_{4}\right) g^{A}\left(t_{4}, t_{2}\right)+\Sigma^{R}\left(t_{3}, t_{4}\right) G_{A}^{\gtrless}\left(t_{4}, t_{2}\right)\right\} .
\end{aligned}
$$

This is not of course an explicit expression for $G_{R}^{\gtrless}$ but a rather complicated integral equation. Among other things, it contains the advanced correlation functions $G_{A}^{\gtrless}$ and the 
cross Green's function. We can derive an analogous equation for $G_{A}^{\gtrless}$ following the same procedure as before. Differentiating $G_{A}^{\gtrless}\left(t_{1}, t_{2}\right)$ with respect to $t_{2}$ and then eliminating the time derivative $\partial g^{\gtrless}\left(t_{1}, t_{2}\right) / \partial t_{2}$ with the help of Eq. (4.15), after some algebra we obtain

$$
\begin{aligned}
& G_{A}^{\gtrless}\left(t_{1}, t_{2}\right)=-i g^{\gtrless}\left(t_{1}, t_{1}\right) g^{A}\left(t_{1}, t_{2}\right)+\int_{t_{1}}^{t_{2}} d t_{3} \mathcal{G}^{<}\left(t_{1}\right) \mathcal{K}^{>}\left(t_{3}\right) g^{A}\left(t_{3}, t_{2}\right) \\
& \quad+\int_{t_{1}}^{t_{2}} d t_{3} \int_{t_{0}}^{\infty} d t_{4}\left\{g^{R}\left(t_{1}, t_{4}\right) \Sigma^{\gtrless}\left(t_{4}, t_{2}\right)+G_{R}^{\gtrless}\left(t_{1}, t_{4}\right) \Sigma^{A}\left(t_{4}, t_{3}\right)\right\} g^{A}\left(t_{3}, t_{2}\right) .
\end{aligned}
$$

In comparison with equations for $G_{R / A}^{\gtrless}$ derived by Lipavský et al. [21], the above equations contain new terms (the second terms on the right-hand sides) which stem from initial correlations. It should also be noted that the self-energies $\Sigma^{\gtrless}$ and $\Sigma^{A}$ are modified in the presence of initial correlations. Physically, the explicit contributions from initial correlations as well as the corresponding corrections to the self-energies are expected to go to zero in the limit $t_{0} \rightarrow-\infty$, since a many-particle system "forgets" details of its initial state.

Before discussing Eqs. (5.6) and (5.7), let us derive analogous equations for the cross Green's functions. First of all we note that the solution to Eqs. (4.24) and (4.27) is

$$
\mathcal{G}^{<}=i g^{R} \mathcal{K}^{<} \mathcal{G}+\mathcal{G}_{\text {hom }}^{<}, \quad \mathcal{G}^{>}=i \mathcal{G} \mathcal{K}^{>} g^{A}+\mathcal{G}_{\text {hom }}^{>},
$$

where $\mathcal{G}_{\text {hom }}^{\gtrless}$ satisfy the homogeneous equations

$$
\left(g_{0}^{-1}-\Sigma^{R}\right) \mathcal{G}_{\text {hom }}^{<}=0, \quad \mathcal{G}_{\text {hom }}^{>}\left(g_{0}^{-1}-\Sigma^{A}\right)=0,
$$

which are to be solved with the boundary conditions (3.23) and (3.24) for $\mathcal{G}^{<}\left(r_{1} t_{0}, r_{1}^{\prime} x_{1}^{\prime}\right)$ and $\mathcal{G}^{>}\left(r_{1} x_{1}, r_{1}^{\prime} t_{0}\right)$. Using the properties of the retarded and advanced Green's functions,

$$
\lim _{t_{1} \rightarrow t_{2} \pm 0} g^{R / A}\left(r_{1} t_{1}, r_{2} t_{2}\right)=\mp i \delta\left(r_{1}-r_{2}\right),
$$

and denoting only the time arguments, we may write the solution to Eqs. (5.9) in the form

$$
\mathcal{G}_{\text {hom }}^{<}\left(t_{1}\right)=i g^{R}\left(t_{1}, t_{0}\right) \mathcal{G}^{<}\left(t_{0}\right), \quad \mathcal{G}_{\text {hom }}^{>}\left(t_{1}\right)=-i \mathcal{G}^{>}\left(t_{0}\right) g^{A}\left(t_{0}, t_{1}\right) .
$$

Inserting these results into Eqs. (5.8) and going to a more transparent notation, we obtain

$$
\begin{aligned}
& \mathcal{G}^{<}\left(t_{1}\right)=i g^{R}\left(t_{1}, t_{0}\right) \mathcal{G}^{<}\left(t_{0}\right)+i \int_{t_{0}}^{t_{1}} d t_{2} g^{R}\left(t_{1}, t_{2}\right) \mathcal{K}^{<}\left(t_{2}\right) \mathcal{G} \\
& \mathcal{G}^{>}\left(t_{1}\right)=-i \mathcal{G}^{>}\left(t_{0}\right) g^{A}\left(t_{0}, t_{1}\right)+i \int_{t_{0}}^{t_{1}} d t_{2} \mathcal{G} \mathcal{K}^{>}\left(t_{2}\right) g^{A}\left(t_{2}, t_{1}\right)
\end{aligned}
$$

It is interesting to note that substitution of these expressions into Eqs. (5.6) and (5.7) allows one to eliminate the cross Green's functions, since the $\mathcal{G}^{\gtrless}\left(t_{0}\right)$ are determined by the initial ensemble, as seen from the boundary conditions (3.23). However, this is not 
a complete solution of the problem because the cross Green's functions enter into the self-energies $\mathcal{K}^{\gtrless}$.

The GKB ansatz [21] follows from Eqs. (5.6) and (5.7) if only the first terms on their right-hand sides are retained. In that case, using Eqs. (3.6) and (5.3), we obtain

$$
\begin{aligned}
& g^{>}\left(t_{1}, t_{2}\right)=g^{R}\left(t_{1}, t_{2}\right) \varrho_{1}^{>}\left(t_{2}\right)-\varrho_{1}^{>}\left(t_{1}\right) g^{A}\left(t_{1}, t_{2}\right), \\
& g^{<}\left(t_{1}, t_{2}\right)=\eta\left(g^{R}\left(t_{1}, t_{2}\right) \varrho_{1}^{<}\left(t_{2}\right)-\varrho_{1}^{<}\left(t_{1}\right) g^{A}\left(t_{1}, t_{2}\right)\right) .
\end{aligned}
$$

Recently the same ansatz has been recovered by approximate solution of the quantum hierarchy for reduced density matrices [11]. The GKB ansatz is exact in the Hartree-Fock approximation for the self-energies and removes some defects of the conventional $\mathrm{KB}$ ansatz [21, 31]. It should be particularly emphasized that the GKB ansatz is consistent with the exact relation (4.12) and, consequently, preserves the correct spectral properties of microscopic dynamics. Nevertheless, the range of validity of the GKB ansatz is not yet known with certainty because it is very difficult to make a general estimate of the last terms in Eqs. (5.6) and (5.7). Qualitative physical arguments [21] suggest that these terms may be neglected if there exist two well-separated time scales characterized by the "collision time" $\tau_{c}$ and the quasiparticle lifetime $\tau$. Then the GKB ansatz provides a reasonable approximation for the correlation functions in the collision integral if $\tau \gg \tau_{c}$, i.e., not far beyond the quasiparticle picture. Iteration of the integral equations (5.6) and (5.7) around the GKB ansatz allows one to find $G_{R / A}^{\gtrless}$ as functionals of the diagonal parts of the correlation functions, $g^{\gtrless}(t, t)$, to some order in $\tau_{c} / \tau$. Note, however, that such a procedure can have consequences for some important properties of the kinetic equation, say for the conservation laws. Later we shall return to this point.

In direct analogy to the GKB ansatz for real-time correlation functions $g^{\gtrless}$, one may expect that keeping only the first terms on the right-hand sides of Eqs. (5.12) is a reasonable ansatz for the cross Green's functions if the description of the system does not go far beyond the quasiparticle picture. Thus, the simple ansatz for the cross Green's functions reads

$$
\mathcal{G}^{<}(t)=i g^{R}\left(t, t_{0}\right) \mathcal{G}^{<}\left(t_{0}\right), \quad \mathcal{G}^{>}(t)=-i \mathcal{G}^{>}\left(t_{0}\right) g^{A}\left(t_{0}, t\right) .
$$

The advantages of this ansatz for the cross Green's functions are the same as those of the GKB ansatz (5.13): (i) it preserves causality and describes fading memory associated with quasiparticle propagation, (ii) effects of strong external fields can be included in a consistent manner, (iii) it is not connected with a quasiclassical approximation. The validity of this ansatz, as well as the validity of the GKB ansatz for $g^{\gtrless}$, must be checked in each specific situation by using the exact integral equations (5.6), (5.7), and (5.12). These equations can also be used to find corrections to $g^{\gtrless}$ and $\mathcal{G}^{\gtrless}$ by iteration.

\section{T-MATRIX APPROXIMATION}

So far we have discussed general consequences of the Dyson equation for the mixed Green's function, which do not depend on the form of the Hamiltonian $\hat{H}$ and the entropy 
operator $\hat{S}$. In order to proceed beyond these formal results, we will consider the special case where the matrix self-energy $\underline{\Sigma}$ is given by Eq. (4.29). Then we can obtain different approximations for the components of the self-energy by determining approximate forms for the two-particle mixed Green's function $\underline{G}^{(2)}$.

In this paper we restrict ourselves to a much used approximation of the many-particle theory, namely the $T$-matrix approximation. In the context of real-time dynamics, this approximation is quite suitable for treating short-range interactions between particles. Another important point is that the $T$-approximation satisfies general criteria for the conservation laws [28]. The applicability of this approximation to the thermodynamic component of $\underline{G}^{(2)}$ depends on whether the initial correlations can be described in terms of binary correlations. For simplicity, we shall assume that the $T$-approximation may be applied to all components of the two-particle Green's function $\underline{G}^{(2)}$ appearing in Eq. (4.29), although in some cases it would be more reasonable to use different approximations for its real-time and thermodynamic components.

\section{A. The T-matrix on the extended contour}

In the T-approximation, the two-particle Green's function is obtained by performing a ladder-type summation in the particle-particle channel [18, 28], which leads to the equation

$$
\begin{aligned}
\underline{G}^{(2)}\left(12,1^{\prime} 2^{\prime}\right)= & \left\{\underline{G}\left(1,1^{\prime}\right) \underline{G}\left(2,2^{\prime}\right)+\eta \underline{G}\left(1,2^{\prime}\right) \underline{G}\left(2,1^{\prime}\right)\right\} \\
& +i \underline{G}\left(1,1^{\prime \prime}\right) \underline{G}\left(2,2^{\prime \prime}\right) \underline{V}\left(1^{\prime \prime} 2^{\prime \prime}, 1^{\prime \prime \prime} 2^{\prime \prime \prime}\right) \underline{G} \underline{G}^{(2)}\left(1^{\prime \prime \prime} 2^{\prime \prime \prime}, 1^{\prime} 2^{\prime}\right),
\end{aligned}
$$

where repeated arguments are summed over single-particle quantum numbers and integrated over the contour $\underline{C}$ shown in Fig. 3. The $\underline{T}$-matrix on the contour $\underline{C}$ is introduced through equations

$$
\begin{aligned}
& \underline{T}\left(12,1^{\prime} 2^{\prime}\right)=\underline{V}\left(12,1^{\prime} 2^{\prime}\right)+i \underline{V}\left(12,1^{\prime \prime} 2^{\prime \prime}\right) \underline{G}\left(1^{\prime \prime}, 2^{\prime \prime \prime}\right) \underline{G}\left(2^{\prime \prime}, 2^{\prime \prime \prime}\right) \underline{T}\left(1^{\prime \prime \prime} 2^{\prime \prime \prime}, 1^{\prime} 2^{\prime}\right), \\
& \underline{T}\left(12,1^{\prime} 2^{\prime}\right)=\underline{V}\left(12,1^{\prime} 2^{\prime}\right)+i \underline{T}\left(12,1^{\prime \prime} 2^{\prime \prime}\right) \underline{G}\left(1^{\prime \prime}, 1^{\prime \prime \prime}\right) \underline{G}\left(2^{\prime \prime}, 2^{\prime \prime \prime}\right) \underline{V}\left(1^{\prime \prime \prime} 2^{\prime \prime \prime}, 1^{\prime} 2^{\prime}\right) .
\end{aligned}
$$

In the condensed matrix notation, Eqs. 6.1) - (6.3) read

$$
\begin{aligned}
& \underline{G}^{(2)}=(\underline{G} \otimes \underline{G})_{\mathrm{ex}}+i(\underline{G} \otimes \underline{G}) \underline{V} \underline{G}^{(2)}, \\
& \underline{T}=\underline{V}+i \underline{V}(\underline{G} \otimes \underline{G}) \underline{T}, \\
& \underline{T}=\underline{V}+i \underline{T}(\underline{G} \otimes \underline{G}) \underline{V},
\end{aligned}
$$

where $\otimes$ stands for the direct product of matrices, and the index "ex" indicates the symmetrized $(\eta=1)$ or anti-symmetrized $(\eta=-1)$ matrix [cf. Eq. (6.1)].

Equation (6.4) can be solved by iteration to yield the relation between the two-particle mixed Green's function and the $\underline{T}$-matrix:

$$
\underline{G}^{(2)}=(\underline{G} \otimes \underline{G})_{\mathrm{ex}}+i(\underline{G} \otimes \underline{G}) \underline{\widetilde{T}}(\underline{G} \otimes \underline{G}),
$$


where the symmetrized (anti-symmetrized) $\underline{T}$-matrix is denoted by

$$
\underline{\widetilde{T}}\left(12,1^{\prime} 2^{\prime}\right)=\underline{T}\left(12,1^{\prime} 2^{\prime}\right)+\eta \underline{T}\left(12,2^{\prime} 1^{\prime}\right)=\underline{T}\left(12,1^{\prime} 2^{\prime}\right)+\eta \underline{T}\left(21,1^{\prime} 2^{\prime}\right) .
$$

Recalling the definition (4.30) of $\underline{V}$, it is easy to see from Eqs. (6.5) and (6.6) that the components $\underline{T}\left(12,1^{\prime} 2^{\prime}\right)$ of the $\underline{T}$-matrix are not zero if only the arguments 1 and 2 correspond to the same part of the contour $\underline{C}$; this is also true for the arguments $1^{\prime}$ and $2^{\prime}$. Taking this into account, the $\underline{T}$-matrix is expressed as

$$
\underline{T}\left(12,1^{\prime} 2^{\prime}\right)=\left\{\begin{array}{lll}
T\left(12,1^{\prime} 2^{\prime}\right) & 1,2 \in C, & 1^{\prime}, 2^{\prime} \in C, \\
\mathcal{T}<\left(12,1^{\prime} 2^{\prime}\right) & 1,2 \in C, & 1^{\prime}, 2^{\prime} \in C_{x}, \\
\mathcal{T}>\left(12,1^{\prime} 2^{\prime}\right) & 1,2 \in C_{x}, & 1^{\prime}, 2^{\prime} \in C, \\
i \mathcal{T}\left(12,1^{\prime} 2^{\prime}\right) & 1,2 \in C_{x}, & 1^{\prime}, 2^{\prime} \in C_{x} .
\end{array}\right.
$$

The real-time $T$-matrix, $T\left(12,1^{\prime} 2^{\prime}\right)$, has four components which can be put into a $2 \times 2$ matrix

$$
T=\left(\begin{array}{cc}
T^{++} & T^{+-} \\
T^{-+} & T^{--}
\end{array}\right)=\left(\begin{array}{cc}
T^{c} & T^{<} \\
T^{>} & T^{a}
\end{array}\right),
$$

where the superscripts $(+/-)$ indicate the branch of the Keldysh contour for the pair of time arguments. In the last line of Eq. (6.9), $\mathcal{T}$ is the $T$-matrix associated with the "imaginary evolution" on $C_{x}$. Finally, we note that there are two cross components of the $\underline{T \text {-matrix, }} \mathcal{T}^{\gtrless}$, which relate the time evolution and initial correlations.

The real-time components of Eqs. 6.5) and (6.6) yield for $T$ the equations

$$
\begin{aligned}
& T=V+i V(G \otimes G) T+i V\left(\mathcal{G}^{<} \otimes \mathcal{G}^{<}\right) \mathcal{T}^{>}, \\
& T=V+i T(G \otimes G) V+i \mathcal{T}^{<}\left(\mathcal{G}^{>} \otimes \mathcal{G}^{>}\right) V
\end{aligned}
$$

which differ from the analogous equations in the standard Green's function formalism by the last terms arising from initial correlations. These terms contain the cross $T$-matrices $\mathcal{T}^{\gtrless}$ and, hence, Eqs. (6.11) and (6.12) are not closed. Thus, though our main interest is with the real-time $T$-matrix defined on the Keldysh contour, we have to consider equations for the other components of the full $\underline{T}$-matrix.

By writing Eqs. (6.5) and (6.6) for $\underline{T}\left(12,1^{\prime} 2^{\prime}\right)$ with $1,2 \in C_{x}$ and $1^{\prime}, 2^{\prime} \in C_{x}$, we find that the "thermodynamic" $T$-matrix satisfies the equations

$$
\mathcal{T}=\mathcal{V}+\mathcal{V}(\mathcal{G} \otimes \mathcal{G}) \mathcal{T}, \quad \mathcal{T}=\mathcal{V}+\mathcal{T}(\mathcal{G} \otimes \mathcal{G}) \mathcal{V}
$$

where no real-time quantities appear, as it must be.

In order to derive equations for the cross $T$-matrices, we make use of Eq. (6.2), where $1,2 \in C_{x}$ and $1^{\prime}, 2^{\prime} \in C$. Then, in the matrix notation, we obtain

$$
\mathcal{T}^{>}=\mathcal{V}(\mathcal{G} \otimes \mathcal{G}) \mathcal{T}^{>}-\mathcal{V}\left(\mathcal{G}^{>} \otimes \mathcal{G}^{>}\right) T
$$

Similarly, taking $1,2 \in C$ and $1^{\prime}, 2^{\prime} \in C_{x}$ in Eq. (6.3) yields

$$
\mathcal{T}^{<}=\mathcal{T}^{<}(\mathcal{G} \otimes \mathcal{G}) \mathcal{V}-T\left(\mathcal{G}^{<} \otimes \mathcal{G}^{<}\right) \mathcal{V}
$$


The above equations can be solved formally for the cross $T$-matrices to give

$$
\begin{aligned}
& \mathcal{T}^{>}=-[I-\mathcal{V}(\mathcal{G} \otimes \mathcal{G})]^{-1} \mathcal{V}\left(\mathcal{G}^{>} \otimes \mathcal{G}^{>}\right) T, \\
& \mathcal{T}^{<}=-T\left(\mathcal{G}^{<} \otimes \mathcal{G}^{<}\right) \mathcal{V}[I-(\mathcal{G} \otimes \mathcal{G}) \mathcal{V}]^{-1},
\end{aligned}
$$

where $I$ is the identity matrix. To simplify these expressions, we make use of Eqs. (6.13), from which it follows that

$$
[I-\mathcal{V}(\mathcal{G} \otimes \mathcal{G})]^{-1} \mathcal{V}=\mathcal{V}[I-(\mathcal{G} \otimes \mathcal{G}) \mathcal{V}]^{-1}=\mathcal{T} .
$$

Now one sees that the cross $T$-matrices (6.16) can be written in the form

$$
\mathcal{T}^{<}=-T\left(\mathcal{G}^{<} \otimes \mathcal{G}^{<}\right) \mathcal{T}, \quad \mathcal{T}^{>}=-\mathcal{T}\left(\mathcal{G}^{>} \otimes \mathcal{G}^{>}\right) T .
$$

These expressions allow us to eliminate the cross $T$-matrices in Eqs. (6.11) and (6.12). Then we get for the real-time $T$-matrix the following equations:

$$
\begin{aligned}
T & =V+i V\left[(G \otimes G)-\left(\mathcal{G}^{<} \otimes \mathcal{G}^{<}\right) \mathcal{T}\left(\mathcal{G}^{>} \otimes \mathcal{G}^{>}\right)\right] T, \\
T & =V+i T\left[(G \otimes G)-\left(\mathcal{G}^{<} \otimes \mathcal{G}^{<}\right) \mathcal{T}\left(\mathcal{G}^{>} \otimes \mathcal{G}^{>}\right)\right] V .
\end{aligned}
$$

As well as being compact, they illustrate the physics of short-time processes in the presence of initial correlations. Let us for a moment suppose that the second terms in square brackets are omitted. Then Eqs. (6.19) and (6.20) will describe binary collisions, $(G \otimes G)$ being a two-particle propagator in a medium. This picture of binary collisions is adequate at the stage of the evolution where initial correlations have been damped. The structure of the full Eqs. (6.19) and (6.20) suggests that initial correlations may be regarded as an additional source of scattering with the corresponding $T$-matrix represented by $\mathcal{T}$ and the propagators represented by the cross Green's functions. It should be noted, however, that the "correlation scattering" described by $\mathcal{T}$ and the dynamical scattering described by the interaction matrix $V$ proceed concurrently, which can be seen by iterating Eqs. (6.19) and (6.20).

It is convenient to reformulate Eqs. (6.19) and (6.20) in terms of quantities which reflect more clearly the two-particle character of the $T$-matrix approximation. First of all, recalling the definition (4.31) of $V\left(12,1^{\prime} 2^{\prime}\right)$, we check by iteration that Eqs. (6.19) and (6.20) imply the following structure of $T\left(12,1^{\prime} 2^{\prime}\right)$ :

$$
T\left(12,1^{\prime} 2^{\prime}\right)=\left\langle r_{1} r_{2}\left|T_{12}\left(t_{1}, t_{1}^{\prime}\right)\right| r_{1}^{\prime} r_{2}^{\prime}\right\rangle \delta_{C}\left(t_{1}-t_{2}\right) \delta_{C}\left(t_{1}^{\prime}-t_{2}^{\prime}\right) .
$$

This formula may serve as a definition of an operator $T_{12}\left(t_{1}, t_{1}^{\prime}\right)$ which will be referred to as the two-particle real-time $T$-matrix. Similarly, we introduce the two-particle thermodynamic $T$-matrix, $\mathcal{T}_{12}\left(x_{1}, x_{1}^{\prime}\right)$, and the interaction operator, $V_{12}$, through the relations

$$
\begin{aligned}
& \mathcal{T}\left(12,1^{\prime} 2^{\prime}\right)=\left\langle r_{1} r_{2}\left|\mathcal{T}_{12}\left(x_{1}, x_{1}^{\prime}\right)\right| r_{1}^{\prime} r_{2}^{\prime}\right\rangle \delta\left(x_{1}-x_{2}\right) \delta\left(x_{1}^{\prime}-x_{2}^{\prime}\right), \\
& V\left(12,1^{\prime} 2^{\prime}\right)=\left\langle r_{1} r_{2}\left|V_{12}\right| r_{1}^{\prime} r_{2}^{\prime}\right\rangle \delta_{C}\left(t_{1}-t_{2}\right) \delta_{C}\left(t_{1}-t_{1}^{\prime}\right) \delta_{C}\left(t_{1}^{\prime}-t_{2}^{\prime}\right) .
\end{aligned}
$$


A comparison with Eq. (4.31) shows that the matrix elements of $V_{12}$ coincide with the interaction amplitudes in the Hamiltonian (2.1). Equation (6.19) is written in terms of the above operators (denoting only the time arguments) as

$$
T_{12}\left(t, t^{\prime}\right)=V_{12} \delta_{C}\left(t-t^{\prime}\right)+i \int_{C} d t^{\prime \prime} V_{12}\left\{G_{12}\left(t, t^{\prime \prime}\right)-\mathcal{G}_{12}^{<}(t) \mathcal{T}_{12} \mathcal{G}_{12}^{>}\left(t^{\prime \prime}\right)\right\} T_{12}\left(t^{\prime \prime}, t^{\prime}\right),
$$

where we have introduced the real-time and cross Green's functions describing two independent particles:

$$
\begin{aligned}
& G_{12}\left(t, t^{\prime}\right)=G_{1}\left(t, t^{\prime}\right) G_{2}\left(t, t^{\prime}\right), \\
& \mathcal{G}_{12}^{\gtrless}(t)=\mathcal{G}_{1}^{\gtrless}(t) \mathcal{G}_{2}^{\gtrless}(t) .
\end{aligned}
$$

It goes without saying that Eq. (6.24) is a matrix equation since each of the arguments, $t$ and $t^{\prime}$, can be taken on either of the branches of the Keldysh contour $C$. Therefore it makes sense to introduce two-particle operators $T_{12}^{\gtrless}, T_{12}^{c}$, and $T_{12}^{a}$, which are defined in the time interval $t_{0} \leq t<\infty$. Again, it is more convenient to use the retarded $\left(T_{12}^{R}\right)$ and advanced $\left(T_{12}^{A}\right)$ operators instead of the chronological and anti-chronological ones. Following the usual rules [28] and recalling that functions $\mathcal{G}^{\gtrless}(t)$ do not depend on whether $t$ is taken on the branch $C^{+}$or on the branch $C^{-}$of the Keldysh contour, Eq. (6.24) is converted into the set of equations

$$
\begin{gathered}
T_{12}^{R / A}\left(t, t^{\prime}\right)=V_{12} \delta\left(t-t^{\prime}\right)+i \int_{t_{0}}^{\infty} d t^{\prime \prime} V_{12} F_{12}^{R / A}\left(t, t^{\prime \prime}\right) T_{12}^{R / A}\left(t^{\prime \prime}, t^{\prime}\right), \\
T_{12}^{\gtrless}\left(t, t^{\prime}\right)=i \int_{t_{0}}^{\infty} d t^{\prime \prime} d t^{\prime \prime \prime} T_{12}^{R}\left(t, t^{\prime \prime}\right)\left\{g_{12}^{\gtrless}\left(t^{\prime \prime}, t^{\prime \prime \prime}\right)-\mathcal{G}_{12}^{<}\left(t^{\prime \prime}\right) \mathcal{T}_{12} \mathcal{G}_{12}^{>}\left(t^{\prime \prime \prime}\right)\right\} T_{12}^{A}\left(t^{\prime \prime \prime}, t^{\prime}\right),
\end{gathered}
$$

where the two-particle Green's functions $g_{12}^{\gtrless}$ and $F_{12}^{R / A}$ are defined as

$$
\begin{aligned}
& g_{12}^{\gtrless}\left(t, t^{\prime}\right)=g_{1}^{\gtrless}\left(t, t^{\prime}\right) g_{2}^{\gtrless}\left(t, t^{\prime}\right), \\
& F_{12}^{R / A}\left(t, t^{\prime}\right)= \pm \theta\left[ \pm\left(t-t^{\prime}\right)\right]\left\{g_{12}^{>}\left(t, t^{\prime}\right)-g_{12}^{<}\left(t, t^{\prime}\right)\right\} .
\end{aligned}
$$

Equation (6.27) describes effective two-particle interactions in a medium. It is similar to the analogous equation in the standard Green's function formalism [18, 28] and goes over to the latter equation in the limit $t_{0} \rightarrow-\infty$. Thus, the influence of initial correlations on the $T$-matrices $T_{12}^{R / A}$ manifests itself only through the short-time behavior of the propagators $F_{12}^{R / A}$. On the other hand, Eq. (6.28), which can be viewed as a generalized optical theorem, contains an explicit contribution from initial correlations, the second term in braces.

It remains to write the cross $T$-matrices $(6.18)$ in terms of two-particle functions. According to the definition of the matrix multiplication, we have

$$
\mathcal{T}_{12}^{<}(t)=-\int_{t_{0}}^{\infty} d t^{\prime} T_{12}^{R}\left(t, t^{\prime}\right) \mathcal{G}_{12}^{<}\left(t^{\prime}\right) \mathcal{T}_{12}, \quad \mathcal{T}_{12}^{>}(t)=-\int_{t_{0}}^{\infty} d t^{\prime} \mathcal{T}_{12} \mathcal{G}_{12}^{>}\left(t^{\prime}\right) T_{12}^{A}\left(t^{\prime}, t\right) .
$$


The "thermodynamic" $T$-matrix, $\mathcal{T}_{12}$, is to be evaluated from Eqs. (6.13). In that case we have to deal only with the problem of correlations in the initial state of the system.

\section{B. Expressions for the self-energies}

Substitution of the two-particle mixed Green's function (6.7) into Eqs. (4.29) yields the expression for the self-energy $\underline{\Sigma}$ in terms of the $\underline{T}$-matrix:

$$
\underline{\Sigma}\left(1,1^{\prime}\right)=i \eta \underline{\widetilde{T}}\left(12,1^{\prime} 2^{\prime}\right) \underline{G}\left(2^{\prime}, 2^{+}\right)=i \eta \underline{G}\left(2^{-}, 2^{\prime}\right) \underline{\widetilde{T}}\left(12^{\prime}, 1^{\prime} 2\right) \text {. }
$$

For its components [see Eq. (4.5)], we have

$$
\begin{aligned}
& \Sigma\left(1,1^{\prime}\right)=i \eta \widetilde{T}\left(12,1^{\prime} 2^{\prime}\right) G\left(2^{\prime}, 2^{+}\right)=i \eta G\left(2^{-}, 2^{\prime}\right) \widetilde{T}\left(12^{\prime}, 1^{\prime} 2\right), \\
& \mathcal{K}^{\gtrless}\left(1,1^{\prime}\right)=i \eta \widetilde{\mathcal{T}}^{\gtrless}\left(12,1^{\prime} 2^{\prime}\right) \mathcal{G}^{\lessgtr}\left(2^{\prime}, 2\right), \\
& \mathcal{K}\left(1,1^{\prime}\right)=\eta \widetilde{\mathcal{T}}\left(12,1^{\prime} 2^{\prime}\right) \mathcal{G}\left(2^{\prime}, 2^{+}\right)=\eta \mathcal{G}\left(2^{-}, 2^{\prime}\right) \widetilde{\mathcal{T}}\left(12^{\prime}, 1^{\prime} 2\right) .
\end{aligned}
$$

The above expression for $\mathcal{K}$, combined with Eqs. (2.18) and (6.13), leads to a closed equation for the thermodynamic Green's function $\mathcal{G}$. However, our prime interest here is with formulae (6.33) and (6.34) which have a direct relationship to the real-time dynamics. Going to the components $\Sigma^{\gtrless}$ and $\Sigma^{R / A}$ of the real-time self-energy, we infer from Eq. (6.33) that

$$
\begin{aligned}
& \Sigma_{1}^{\gtrless}\left(t, t^{\prime}\right)=i \eta \operatorname{Tr}_{(2)} \widetilde{T}_{12}^{\gtrless}\left(t, t^{\prime}\right) g_{2}^{\lessgtr}\left(t^{\prime}, t\right), \\
& \Sigma_{1}^{R / A}\left(t, t^{\prime}\right)=i \eta \underset{(2)}{\operatorname{Tr}}\left\{\widetilde{T}_{12}^{R / A}\left(t, t^{\prime}\right) g_{2}^{<}\left(t^{\prime}, t\right)+\widetilde{T}_{12}^{<}\left(t, t^{\prime}\right) g_{2}^{A / R}\left(t^{\prime}, t\right)\right\} .
\end{aligned}
$$

We now turn to Eq. (6.34). The cross T-matrices can be eliminated by means of Eqs. 6.31). Then we get

$$
\begin{aligned}
& \mathcal{K}_{1}^{<}(t)=-i \eta \int_{t_{0}}^{\infty} d t^{\prime} \underset{(2)}{\operatorname{Tr}}\left\{T_{12}^{R}\left(t, t^{\prime}\right) \mathcal{G}_{12}^{<}\left(t^{\prime}\right) \widetilde{\mathcal{T}}_{12} \mathcal{G}_{2}^{>}(t)\right\}, \\
& \mathcal{K}_{1}^{>}(t)=-i \eta \int_{t_{0}}^{\infty} d t^{\prime} \underset{(2)}{\operatorname{Tr}}\left\{\mathcal{G}_{2}^{<}(t) \widetilde{\mathcal{T}}_{12} \mathcal{G}_{12}^{>}\left(t^{\prime}\right) T_{12}^{A}\left(t^{\prime}, t\right)\right\} .
\end{aligned}
$$

In the special case that the entropy operator contains the one-particle term $\hat{S}^{0}$ only, we have $\mathcal{T}_{12}=0$ and, consequently, the cross components of the self-energy vanish.

Now Eqs. (4.17) - (4.20), (4.24), (4.27), together with the expressions for the selfenergies given in this section, provide a closed description for the evolution of the system in the $T$-matrix approximation. Initial correlations enter into play through the thermodynamic Green function $\mathcal{G}$ and the thermodynamic $T$-matrix $\mathcal{T}_{12}$, which are to be calculated from Eqs. (2.18) and (6.13). Although this scheme is self-consistent, it can only be realized by numerical calculations. To gain a physical picture of short-time dynamics and understand the role of initial correlations, it is reasonable to go over to a simpler description based on a kinetic equation for the one-particle density matrix. In the next section 
we will apply the mixed Green's function formalism to the derivation of explicit kinetic equations.

\section{KINETIC EQUATIONS}

For simplicity, we assume the initial state of the system to be spatially homogeneous. Generalization to inhomogeneous systems is straightforward but adds of course algebraic complexity. In the homogeneous case, it is convenient to work in momentum representation where all matrix elements are defined with respect to normalized single-particle quantum states $|p\rangle=|\mathbf{p}, \sigma\rangle$ in a volume $\Omega$ with periodic boundary conditions. As usual, the limit $\Omega \rightarrow \infty$ must be taken at the end of the calculations. By going from the field operators to the creation and annihilation operators, $a_{p}^{\dagger}$ and $a_{p}$, we find that the oneparticle density matrices $\varrho_{1}^{\gtrless}(t)$, given in the $r$-representation by Eqs. (3.7) and (3.8), now have the elements

$$
\left\langle p_{1}\left|\varrho_{1}^{<}(t)\right| p_{1}^{\prime}\right\rangle=\delta_{p_{1} p_{1}^{\prime}} f_{p_{1}}(t), \quad\left\langle p_{1}\left|\varrho_{1}^{>}(t)\right| p_{1}^{\prime}\right\rangle=\delta_{p_{1} p_{1}^{\prime}} \bar{f}_{p_{1}}(t)
$$

where $f_{p}(t)=\left\langle a_{p H}^{\dagger}(t) a_{p H}(t)\right\rangle$ is the one-particle distribution function and

$$
\bar{f}_{p}(t)=1+\eta f_{p}(t)
$$

For Fermi systems $(\eta=-1), \bar{f}_{p}(t)$ is the distribution function for holes.

\section{A. Short-time kinetic equation in the T-matrix approximation}

Our starting point is the generalized kinetic equation (5.1). Taking its diagonal part with respect to time variables and the $p$-variables, the left-hand side reduces to $\eta \partial f_{p_{1}}(t) / \partial t$. It is convenient to eliminate retarded and advanced functions on the righthand side with the aid of Eq. (4.12) and the relation [18]

$$
\Sigma^{R / A}\left(t, t^{\prime}\right)=\Sigma^{\delta}\left(t, t^{\prime}\right) \pm \theta\left[ \pm\left(t-t^{\prime}\right)\right]\left\{\Sigma^{>}\left(t, t^{\prime}\right)-\Sigma^{<}\left(t, t^{\prime}\right)\right\}
$$

where $\Sigma^{\delta}\left(t, t^{\prime}\right)$ is a singular term in the self-energies. In the spatially homogeneous case, $\Sigma^{\delta}$ does not contribute to the diagonal part of Eq. (5.1), so that we arrive at the equation

$$
\begin{aligned}
\frac{\partial}{\partial t} f_{p_{1}}(t)= & \eta \int_{t_{0}}^{t} d t^{\prime}\left\{g_{p_{1}}^{<}\left(t, t^{\prime}\right) \Sigma_{p_{1}}^{>}\left(t^{\prime}, t\right)+\Sigma_{p_{1}}^{>}\left(t, t^{\prime}\right) g_{p_{1}}^{<}\left(t^{\prime}, t\right)\right\} \\
& -\eta \int_{t_{0}}^{t} d t^{\prime}\left\{g_{p_{1}}^{>}\left(t, t^{\prime}\right) \Sigma_{p_{1}}^{<}\left(t^{\prime}, t\right)+\Sigma_{p_{1}}^{<}\left(t, t^{\prime}\right) g_{p_{1}}^{>}\left(t^{\prime}, t\right)\right\} \\
& +\eta\left\{\mathcal{K}_{p_{1}}^{<}(t) \mathcal{G}_{p_{1}}^{>}(t)-\mathcal{G}_{p_{1}}^{<}(t) \mathcal{K}_{p_{1}}^{>}(t)\right\},
\end{aligned}
$$

where we have taken into account that the Green's functions and the self-energies are diagonal matrices with respect to the one-particle $p$-variables. 
Equation (7.4) is still exact. We now take for the self-energies approximate expressions (6.36) and (6.38). Using also the optical theorem (6.28), the kinetic equation can be written in the form

$$
\frac{\partial}{\partial t} f_{p_{1}}(t)=I_{p_{1}}^{(B)}(t)+I_{p_{1}}^{(C)}(t)
$$

with the collision integrals

$$
I_{p_{1}}^{(B)}(t)=\sum_{p_{2}}\left\langle p_{1} p_{2}\left|I_{12}^{(B)}(t)\right| p_{1} p_{2}\right\rangle, \quad I_{p_{1}}^{(C)}(t)=\sum_{p_{2}}\left\langle p_{1} p_{2}\left|I_{12}^{(C)}(t)\right| p_{1} p_{2}\right\rangle .
$$

The two-particle collision operators, $I_{12}^{(B)}(t)$ and $I_{12}^{(C)}(t)$, are defined as

$$
\begin{aligned}
I_{12}^{(B)}(t) & =\int_{t_{0}}^{t} d t^{\prime}\left\{g_{12}^{>}\left(t, t^{\prime}\right)\left(T_{12}^{R} g_{12}^{<} \widetilde{T}_{12}^{A}\right)\left(t^{\prime}, t\right)+\left(\widetilde{T}_{12}^{R} g_{12}^{<} T_{12}^{A}\right)\left(t, t^{\prime}\right) g_{12}^{>}\left(t^{\prime}, t\right)\right\} \\
& -\int_{t_{0}}^{t} d t^{\prime}\left\{g_{12}^{<}\left(t, t^{\prime}\right)\left(T_{12}^{R} g_{12}^{>} \widetilde{T}_{12}^{A}\right)\left(t^{\prime}, t\right)+\left(\widetilde{T}_{12}^{R} g_{12}^{>} T_{12}^{A}\right)\left(t, t^{\prime}\right) g_{12}^{<}\left(t^{\prime}, t\right)\right\}, \\
I_{12}^{(C)}(t) & =i\left(C_{12} T_{12}^{A}-T_{12}^{R} C_{12}\right)(t, t) \\
+ & \int_{t_{0}}^{t} d t^{\prime}\left\{\left(g_{12}^{<}-g_{12}^{>}\right)\left(t, t^{\prime}\right)\left(T_{12}^{R} C_{12} T_{12}^{A}\right)\left(t^{\prime}, t\right)\right. \\
& \left.\quad+\left(T_{12}^{R} C_{12} T_{12}^{A}\right)\left(t, t^{\prime}\right)\left(g_{12}^{<}-g_{12}^{>}\right)\left(t^{\prime}, t\right)\right\},
\end{aligned}
$$

where we have introduced the two-particle time correlation matrix

$$
C_{12}\left(t, t^{\prime}\right)=\mathcal{G}_{12}^{<}(t) \widetilde{\mathcal{T}}_{12} \mathcal{G}_{12}^{>}\left(t^{\prime}\right)
$$

In writing Eqs. (7.7) and (7.8), we have followed the convention that matrix multiplication like $A B C$ implies integration over all intermediate time arguments.

The structure of $I_{12}^{(B)}(t)$ is similar to the structure of quantum Boltzmann-like collision operators in the $T$-matrix approximation [13, 18, 28]. The new ingredient is the operator $I_{12}^{C}(t)$ which involves the effects of initial correlations through the matrix $C_{12}\left(t, t^{\prime}\right)$. However, this is not the end of the story, since Eqs. (7.7) and (7.8) contain double-time correlation functions $g_{12}^{\gtrless}\left(t, t^{\prime}\right)$ which are to be expressed in terms of the distribution function $f_{p}(t)$ for Eq. (7.5) to become a closed kinetic equation. We also note that the time evolution of the cross correlation functions $\mathcal{G}_{12}^{\gtrless}(t)$ in Eq. (7.9) must be specified. We shall make use of Eqs. (5.13) and (5.14) to complete the kinetic equation. Then we have the following expressions for the two-particle quantities of interest:

$$
\begin{aligned}
& g_{12}^{\gtrless}\left(t, t^{\prime}\right)=g_{12}^{R}\left(t, t^{\prime}\right) \varrho_{1}^{\gtrless}\left(t^{\prime}\right) \varrho_{2}^{\gtrless}\left(t^{\prime}\right)+\varrho_{1}^{\gtrless}(t) \varrho_{2}^{\gtrless}(t) g_{12}^{A}\left(t, t^{\prime}\right), \\
& \mathcal{G}_{12}^{<}(t)=-g_{12}^{R}\left(t, t_{0}\right) \mathcal{G}_{12}^{<}\left(t_{0}\right), \quad \mathcal{G}_{12}^{>}(t)=-\mathcal{G}_{12}^{>}\left(t_{0}\right) g_{12}^{A}\left(t_{0}, t\right),
\end{aligned}
$$

where

$$
g_{12}^{R / A}\left(t, t^{\prime}\right)=g_{1}^{R / A}\left(t, t^{\prime}\right) g_{2}^{R / A}\left(t, t^{\prime}\right)
$$


are two-particle propagators. We show in Appendix E that Eqs. (7.11) provide a convenient way of writing the time correlation matrix (7.9):

$$
C_{12}\left(t, t^{\prime}\right)=g_{12}^{R}\left(t, t_{0}\right) \chi_{12}\left(t_{0}\right) g_{12}^{A}\left(t_{0}, t^{\prime}\right)
$$

Here $\chi_{12}\left(t_{0}\right)$ is the initial two-particle correlation matrix $\chi_{12}(t)$, which is defined as the correlated part of the two-particle density matrix:

$$
\chi_{12}(t)=\varrho_{12}(t)-\left(\varrho_{1}(t) \varrho_{2}(t)\right)_{\mathrm{ex}} .
$$

In momentum representation, the matrix elements of $\varrho_{12}(t)$ are given by

$$
\left\langle p_{1} p_{2}\left|\varrho_{12}(t)\right| p_{1}^{\prime} p_{2}^{\prime}\right\rangle=\left\langle a_{p_{2}^{\prime} H}^{\dagger}(t) a_{p_{1}^{\prime} H}^{\dagger}(t) a_{p_{1} H}(t) a_{p_{2} H}(t)\right\rangle
$$

After inserting the expressions $(\overline{7.10})$ and (7.13) into Eqs. (7.7) and (77.8), the collision integrals can then be written in terms of the one-particle distribution function $f_{p}(t)$, the two-particle $T$-matrices $T_{12}^{R / A}\left(t, t^{\prime}\right)$, and the propagators $g_{p}^{R / A}\left(t, t^{\prime}\right)$. Thus, to obtain a closed kinetic description of short-time dynamics, it remains to consider equations for $T^{R / A}$ and $g^{R / A}$. Due to the properties

$$
\left[g_{p_{1}}^{R}\left(t, t^{\prime}\right)\right]^{*}=g_{p_{1}}^{A}\left(t^{\prime}, t\right), \quad\left\langle p_{1} p_{2}\left|T_{12}^{R}\left(t, t^{\prime}\right)\right| p_{1}^{\prime} p_{2}^{\prime}\right\rangle^{*}=\left\langle p_{1}^{\prime} p_{2}^{\prime}\left|T_{12}^{A}\left(t^{\prime}, t\right)\right| p_{1} p_{2}\right\rangle,
$$

we may dwell on the retarded functions only. Equation for $T_{12}^{R}$ follows directly from Eq. (6.27) if we use the ansatz (7.10) to express $F_{12}^{R}$, given by Eq. (6.30), in terms of the one-particle density matrix. We then obtain

$$
T_{12}^{R}\left(t, t^{\prime}\right)=V_{12} \delta\left(t-t^{\prime}\right)-i \int_{t_{0}}^{t} d t^{\prime \prime} V_{12} g_{12}^{R}\left(t, t^{\prime \prime}\right)\left(\varrho_{1}^{<} \varrho_{2}^{<}-\varrho_{1}^{>} \varrho_{2}^{>}\right)_{t^{\prime \prime}} T_{12}^{R}\left(t^{\prime \prime}, t^{\prime}\right)
$$

Finally, the equation for $g_{1}^{R}\left(t, t^{\prime}\right)$ follows from Eq. (4.14) in momentum representation. Since we consider a homogeneous system, we have

$$
\left(i \frac{\partial}{\partial t}-\varepsilon_{p_{1}}\right) g_{p_{1}}^{R}\left(t, t^{\prime}\right)=\delta\left(t-t^{\prime}\right)+\int_{t_{0}}^{\infty} d t^{\prime \prime} \Sigma_{p_{1}}^{R}\left(t, t^{\prime \prime}\right) g_{p_{1}}^{R}\left(t^{\prime \prime}, t^{\prime}\right)
$$

where $\varepsilon_{p}=|\mathbf{p}|^{2} / 2 m$ is the free-particle energy. The self-energy $\Sigma_{p_{1}}^{R}$ is then found in terms of $f_{p}, T_{12}^{R / A}$, and $g_{p}^{R / A}$ by using Eq. (6.37), the optical theorem (6.28), and the ansatz (5.13). We will not give this somewhat lengthy expression for $\Sigma_{p_{1}}^{R}$.

\section{B. Short-time kinetic equation in Born approximation}

To demonstrate the principal features of the collision integrals in the kinetic equation (7.5), we shall consider the operators (7.7) and (7.8) in Born approximation for the $T$-matrices. For weak interaction, Eq. (7.17) can be solved by iteration. The result in a matrix notation up to second order in $V_{12}$ reads

$$
T_{12}^{R}\left(t, t^{\prime}\right)=V_{12} \delta\left(t-t^{\prime}\right)-i V_{12} g_{12}^{R}\left(t, t^{\prime}\right)\left(\varrho_{1}^{<} \varrho_{2}^{<}-\varrho_{1}^{>} \varrho_{2}^{>}\right)_{t^{\prime}} V_{12}
$$


The analogous result for the advanced $T$-matrix is

$$
T_{12}^{A}\left(t, t^{\prime}\right)=V_{12} \delta\left(t-t^{\prime}\right)+i V_{12}\left(\varrho_{1}^{<} \varrho_{2}^{<}-\varrho_{1}^{>} \varrho_{2}^{>}\right)_{t} g_{12}^{A}\left(t, t^{\prime}\right) V_{12} .
$$

Now, to find the collision integrals in Born approximation, these expressions are to be inserted into Eqs. (7.7) and (7.8). Note that in all terms, except for the first term on the right-hand side of Eq. (7.8), one can take the $T$-matrices to first order in the interaction $V_{12}$. As already discussed, the correlation functions $g_{12}^{\gtrless}$ are eliminated in favor of the one-particle density matrices by means of the ansatz (7.10). Then, recalling Eqs. (7.1) for the elements of the one-particle density matrices, a simple algebra gives for the collision integrals

$$
\begin{gathered}
I_{p_{1}}^{(B)}(t)=-\sum_{p_{2} p_{1}^{\prime} p_{2}^{\prime}} \int_{t_{0}}^{t} d t^{\prime} W_{p_{1} p_{2}, p_{1}^{\prime} p_{2}^{\prime}}^{(B)}\left(t, t^{\prime}\right)\left(f_{p_{1}} f_{p_{2}} \bar{f}_{p_{1}^{\prime}} \bar{f}_{p_{2}^{\prime}}-\bar{f}_{p_{1}} \bar{f}_{p_{2}} f_{p_{1}^{\prime}} f_{p_{2}^{\prime}}\right)_{t^{\prime}}, \\
I_{p_{1}}^{(C)}(t)=2 \sum_{p_{2}} \operatorname{Im}\left\{\left\langle p_{1} p_{2}\left|V_{12} C_{12}(t, t)\right| p_{1} p_{2}\right\rangle\right\} \\
\quad+\sum_{p_{2} p_{1}^{\prime} p_{2}^{\prime}} \int_{t_{0}}^{t} d t^{\prime}\left\{W_{p_{1} p_{2}, p_{1}^{\prime} p_{2}^{\prime}}^{(C)}\left(t, t^{\prime}\right)\left(f_{p_{1}^{\prime}} f_{p_{2}^{\prime}}-\bar{f}_{p_{1}^{\prime}} \bar{f}_{p_{2}^{\prime}}\right)_{t^{\prime}}\right. \\
\left.\quad-W_{p_{1}^{\prime} p_{2}^{\prime}, p_{1} p_{2}}^{(C)}\left(t, t^{\prime}\right)\left(f_{p_{1}} f_{p_{2}}-\bar{f}_{p_{1}} \bar{f}_{p_{2}}\right)_{t^{\prime}}\right\} .
\end{gathered}
$$

The quantities $W^{(B)}$ and $W^{(C)}$ play the role of the transition probabilities or the memory functions and are given by

$$
\begin{aligned}
& W_{p_{1} p_{2}, p_{1}^{\prime} p_{2}^{\prime}}^{(B)}\left(t, t^{\prime}\right)=\left|\left\langle p_{1} p_{2}\left|\widetilde{V}_{12}\right| p_{1}^{\prime} p_{2}^{\prime}\right\rangle\right|^{2} \operatorname{Re}\left\{X_{p_{1} p_{2}, p_{1}^{\prime} p_{2}^{\prime}}^{(B)}\left(t, t^{\prime}\right)\right\}, \\
& W_{p_{1} p_{2}, p_{1}^{\prime} p_{2}^{\prime}}^{(C)}\left(t, t^{\prime}\right)=-2 \operatorname{Re}\left\{X_{p_{1} p_{2}, p_{1}^{\prime} p_{2}^{\prime}}^{(C)}\left(t, t^{\prime}\right)\right\},
\end{aligned}
$$

where

$$
\begin{aligned}
& X_{p_{1} p_{2}, p_{1}^{\prime} p_{2}^{\prime}}^{(B)}\left(t, t^{\prime}\right)=g_{p_{1} p_{2}}^{R}\left(t, t^{\prime}\right) g_{p_{1}^{\prime} p_{2}^{\prime}}^{A}\left(t^{\prime}, t\right), \\
& X_{p_{1} p_{2}, p_{1}^{\prime} p_{2}^{\prime}}^{(C)}\left(t, t^{\prime}\right)=\left\langle p_{1} p_{2}\left|C_{12}\left(t, t^{\prime}\right) V_{12}\right| p_{1}^{\prime} p_{2}^{\prime}\right\rangle g_{p_{1}^{\prime} p_{2}^{\prime}}^{A}\left(t^{\prime}, t\right)\left\langle p_{1}^{\prime} p_{2}^{\prime}\left|V_{12}\right| p_{1} p_{2}\right\rangle .
\end{aligned}
$$

Note that the memory function (7.23) has the symmetry property

$$
W_{p_{1} p_{2}, p_{1}^{\prime} p_{2}^{\prime}}^{(B)}\left(t, t^{\prime}\right)=W_{p_{1}^{\prime} p_{2}^{\prime}, p_{1} p_{2}}^{(B)}\left(t, t^{\prime}\right),
$$

while this is not the case for the memory function (7.24).

A few comments should be made here about the results (7.21) and (7.22). The term $I^{(B)}$ is a Boltzmann-like collision integral, where the memory effects are involved through the retarded and advanced Green's functions. Collision integrals of this type are used extensively in the delayed quantum kinetics (see, e.g., [5] and references therein). The extra 
collision integral, $I^{(C)}$, collects explicit contributions from initial correlations and contains two different terms. The first term in Eq. (7.22) is linear in the interaction and does not depend on the one-particle distribution function. Lee et al. [10] were the first to derive the analogous term for a weakly coupled low-density gas. Recently the first-order correlation term in $I^{(C)}$ was re-derived within the density operator theory [12 and the real-time Green's function formalism in which the initial correlation where specified in the form of a cluster expansion [20]. The remaining integral term in Eq. (7.22) has a "gain-loss" form and gives an account of a relaxation process caused by initial correlations. To the authors knowledge, this term was not considered before in short-time kinetics. Physically, it can be relevant for describing the quasiparticle formation at the first stage of evolution. The most interesting thing about the "gain-loss" correlation term in Eq. (7.22) is that it depends on the nonequilibrium one-particle distribution functions, but its structure is quite different from the structure of the Boltzmann-like collision integral (7.21). Note that, for Fermi systems, the combination of one-particle distribution functions in this

term, $f_{p_{1}} f_{p_{2}}-\bar{f}_{p_{1}} \bar{f}_{p_{2}}=f_{p_{1}} f_{p_{2}}-\left(1-f_{p_{1}}\right)\left(1-f_{p_{2}}\right)$, is nothing but the difference between particle-particle and hole-hole excitations, i.e., the Pauli blocking factor.

\section{Retarded Green's function}

To complete the kinetic equation (7.5), we need to evaluate the retarded Green's function from Eq. (7.18). First of all we find the self-energy $\Sigma_{p_{1}}^{R}\left(t, t^{\prime}\right)$, given by Eq. (6.37), in Born approximation for the $T$-matrices. To eliminate the correlation function $g^{<}$, we make use of the GKB ansatz (5.13). Then we obtain

$$
\Sigma_{p_{1}}^{R}\left(t, t^{\prime}\right)=\Sigma_{p_{1}}^{\mathrm{HF}}(t) \delta\left(t-t^{\prime}\right)+\sum_{p_{2}} \Lambda_{p_{1} p_{2}}\left(t, t^{\prime}\right) g_{p_{2}}^{A}\left(t^{\prime}, t\right)
$$

where

$$
\Sigma_{p_{1}}^{\mathrm{HF}}(t)=\sum_{p_{2}}\left\langle p_{1} p_{2}\left|\widetilde{V}_{12}\right| p_{1} p_{2}\right\rangle f_{p_{2}}(t)
$$

is the Hartree-Fock term and we have defined

$$
\begin{gathered}
\Lambda_{p_{1} p_{2}}\left(t, t^{\prime}\right)=\frac{1}{2} \sum_{p_{1}^{\prime} p_{2}^{\prime}}\left|\left\langle p_{1} p_{2}\left|\widetilde{V}_{12}\right| p_{1}^{\prime} p_{2}^{\prime}\right\rangle\right|^{2} g_{p_{1}^{\prime} p_{2}^{\prime}}^{R}\left(t, t^{\prime}\right)\left(f_{p_{2}} \bar{f}_{p_{1}^{\prime}} \bar{f}_{p_{2}^{\prime}}-\bar{f}_{p_{2}} f_{p_{1}^{\prime}} f_{p_{2}^{\prime}}\right)_{t^{\prime}} \\
+\eta\left\langle p_{1} p_{2}\left|V_{12} C_{12}\left(t, t^{\prime}\right) V_{12}\right| p_{1} p_{2}\right\rangle .
\end{gathered}
$$

Insertion of Eq. (7.28) into Eq. (7.18) leads to the integro-differential equation

$$
\left(i \frac{\partial}{\partial t}-E_{p_{1}}(t)\right) g_{p_{1}}^{R}\left(t, t^{\prime}\right)=\delta\left(t-t^{\prime}\right)+\sum_{p_{2}} \int_{t^{\prime}}^{t} d t^{\prime \prime} \Lambda_{p_{1} p_{2}}\left(t, t^{\prime \prime}\right)\left[g_{p_{2}}^{R}\left(t, t^{\prime \prime}\right)\right]^{*} g_{p_{1}}^{R}\left(t^{\prime \prime}, t^{\prime}\right),
$$

where

$$
E_{p_{1}}(t)=\varepsilon_{p_{1}}+\Sigma_{p_{1}}^{\mathrm{HF}}(t)
$$


is the re-normalized particle energy. Equation (7.31) should to be solved together with the kinetic equation for the one-particle distribution function, but it seems to be a very difficult task, even using numerical methods. We therefore will construct an approximate solution to Eq. (7.31), valid for a weak interaction.

First we note that the retarded Green's function $g_{p_{1}}^{R}\left(t, t^{\prime}\right)$ can always be written in the form

$$
g_{p_{1}}^{R}\left(t, t^{\prime}\right)=-i \theta\left(t-t^{\prime}\right) \exp \left\{-i \omega_{p_{1}}\left(t, t^{\prime}\right)-\Phi_{p_{1}}\left(t, t^{\prime}\right)\right\}
$$

where

$$
\omega_{p_{1}}\left(t, t^{\prime}\right)=\int_{t^{\prime}}^{t} d t^{\prime \prime} E_{p_{1}}\left(t^{\prime \prime}\right)=\varepsilon_{p_{1}}\left(t-t^{\prime}\right)+\int_{t^{\prime}}^{t} d t^{\prime \prime} \Sigma_{p_{1}}^{\mathrm{HF}}\left(t^{\prime \prime}\right),
$$

and $\Phi_{p_{1}}\left(t, t^{\prime}\right)$ is some complex-valued function. It satisfies the obvious initial condition $\Phi_{p}(t, t)=0$ and has a simple physical interpretation. Its real part determines the quasiparticle damping, while the imaginary part can be associated with higher-order corrections to the quasiparticle energy. Inserting the expression (7.33) into Eq. (7.31), one obtains for $\Phi_{p_{1}}\left(t, t^{\prime}\right)$ an integro-differential equation, which can then be recast into the following integral equation:

$$
\Phi_{p_{1}}\left(t, t^{\prime}\right)=-\sum_{p_{2}} \int_{t^{\prime}}^{t} d \tau_{1} \int_{t^{\prime}}^{\tau_{1}} d \tau_{2} \mathrm{e}^{i \omega_{p_{1} p_{2}}\left(\tau_{1}, \tau_{2}\right)} \mathrm{e}^{\left[\Phi_{p_{1}}\left(\tau_{1}, t^{\prime}\right)-\Phi_{p_{1}}\left(\tau_{2}, t^{\prime}\right)-\Phi_{p_{2}}\left(\tau_{1}, \tau_{2}\right)\right]} \Lambda_{p_{1} p_{2}}\left(\tau_{1}, \tau_{2}\right),
$$

where

$$
\omega_{p_{1} p_{2}}\left(t, t^{\prime}\right)=\omega_{p_{1}}\left(t, t^{\prime}\right)+\omega_{p_{2}}\left(t, t^{\prime}\right)
$$

Since the right-hand side of Eq. (7.35) is already of second order in the interaction, the leading approximation for $\Phi_{p}\left(t, t^{\prime}\right)$ can be obtained by setting $\Phi=0$ in the integrand. In the weak coupling case, the imaginary part of $\Phi_{p}\left(t, t^{\prime}\right)$ is small compared with $\omega_{p}\left(t, t^{\prime}\right)$, so that our interest is in the real part of $\Phi_{p}\left(t, t^{\prime}\right)$ describing the quasiparticle damping. With Eq. (7.30), we find

$$
\begin{aligned}
\Gamma_{p_{1}}\left(t, t^{\prime}\right)= & \operatorname{Re}\left\{\Phi_{p_{1}}\left(t, t^{\prime}\right)\right\} \\
= & \frac{1}{2} \sum_{p_{2} p_{1}^{\prime} p_{2}^{\prime}} \int_{t^{\prime}}^{t} d \tau_{1} \int_{t^{\prime}}^{\tau_{1}} d \tau_{2}\left|\left\langle p_{1} p_{2}\left|\widetilde{V}_{12}\right| p_{1}^{\prime} p_{2}^{\prime}\right\rangle\right|^{2} \\
& \times \cos \left[\Delta \omega_{p_{1} p_{2}, p_{1}^{\prime} p_{2}^{\prime}}\left(\tau_{1}, \tau_{2}\right)\right]\left(f_{p_{2}} \bar{f}_{p_{1}^{\prime}} \bar{f}_{p_{2}^{\prime}}-\bar{f}_{p_{2}} f_{p_{1}^{\prime}} f_{p_{2}^{\prime}}\right)_{\tau_{2}} \\
& -\eta \sum_{p_{2}} \int_{t^{\prime}}^{t} d \tau_{1} \int_{t^{\prime}}^{\tau_{1}} d \tau_{2} \operatorname{Re}\left\{\mathrm{e}^{i \omega_{p_{1} p_{2}}\left(\tau_{1}, \tau_{2}\right)}\left\langle p_{1} p_{2}\left|V_{12} C_{12}\left(\tau_{1}, \tau_{2}\right) V_{12}\right| p_{1} p_{2}\right\rangle\right\},
\end{aligned}
$$

where

$$
\Delta \omega_{p_{1} p_{2}, p_{1}^{\prime} p_{2}^{\prime}}=\omega_{p_{1} p_{2}}\left(t, t^{\prime}\right)-\omega_{p_{1}^{\prime} p_{2}^{\prime}}\left(t, t^{\prime}\right)
$$

The first term in the expression (7.37) is long-lived because a substantial contribution to the inner integral is made by the region $\left(\tau_{1}-\tau_{2}\right)$ of the order of the collision duration 
time $\tau_{c}$ and, consequently, the first term grows with $\left(t-t^{\prime}\right)$ if $\left(t-t^{\prime}\right) \gg \tau_{c}$. The behavior of the second term depends critically on the time $\tau_{\text {in }}$ required for damping of the initial correlations. Since the matrix elements of $C_{12}\left(t, t^{\prime}\right)$ according to Eq. (7.13) are oscillating functions of $t-t_{0}$ and $t^{\prime}-t_{0}$, the second term in Eq. (7.37) is expected to have a plateau at $\left(t-t_{0}\right) \gg \tau_{i n}$ and $\left(t^{\prime}-t_{0}\right) \gg \tau_{i n}$. The interplay between the "quasiparticle" and "correlation" contributions to $\Gamma_{p}\left(t, t^{\prime}\right)$ at the initial stage of the evolution can be of interest in short-time kinetics. However, a systematic analysis of this point requires more detailed information on the interaction operator $V_{12}$ and the initial two-particle correlation matrix $\chi_{12}\left(t_{0}\right)$.

To summarize, we may conclude that a reasonable approximation for the Green's functions in Eqs. (7.13), (7.25), and (7.26) is given by

$$
g_{p_{1}}^{R}\left(t, t^{\prime}\right)=\left[g_{p_{1}}^{A}\left(t^{\prime}, t\right)\right]^{*}=-i \theta\left(t-t^{\prime}\right) \exp \left\{-i \omega_{p_{1}}\left(t, t^{\prime}\right)-\Gamma_{p_{1}}\left(t, t^{\prime}\right)\right\} .
$$

This retarded Green's function satisfies the equation

$$
\left(i \frac{\partial}{\partial t}-E_{p_{1}}(t)+i \gamma_{p_{1}}\left(t, t^{\prime}\right)\right) g_{p}^{R}\left(t, t^{\prime}\right)=\delta\left(t-t^{\prime}\right)
$$

with the time-dependent damping

$$
\begin{aligned}
& \gamma_{p_{1}}\left(t, t^{\prime}\right)=\frac{\partial}{\partial t} \Gamma_{p_{1}}\left(t, t^{\prime}\right) \\
& =\frac{1}{2} \sum_{p_{2} p_{1}^{\prime} p_{2}^{\prime}} \int_{t^{\prime}}^{t} d \tau\left|\left\langle p_{1} p_{2}\left|\widetilde{V}_{12}\right| p_{1}^{\prime} p_{2}^{\prime}\right\rangle\right|^{2} \cos \left[\Delta \omega_{p_{1} p_{2}, p_{1}^{\prime} p_{2}^{\prime}}(t, \tau)\right]\left(f_{p_{2}} \bar{f}_{p_{1}^{\prime}} \bar{f}_{p_{2}^{\prime}}-\bar{f}_{p_{2}} f_{p_{1}^{\prime}} f_{p_{2}^{\prime}}\right)_{\tau} \\
& \quad-\eta \sum_{p_{2}} \int_{t^{\prime}}^{t} d \tau \operatorname{Re}\left\{\mathrm{e}^{i \omega_{p_{1} p_{2}}(t, \tau)}\left\langle p_{1} p_{2}\left|V_{12} C_{12}(t, \tau) V_{12}\right| p_{1} p_{2}\right\rangle\right\} .
\end{aligned}
$$

Equation (7.40) can be considered as an approximate version of Eq. (7.31).

\section{Memory effects and energy conservation}

The expressions (7.39) for the retarded and advanced Green's functions with $\omega_{p_{1}}\left(t, t^{\prime}\right)$ and $\Gamma_{p_{1}}\left(t, t^{\prime}\right)$ given respectively by Eq. (7.34) and Eq. (7.37) complete the kinetic equation since the memory functions in the collision integral, as well as the matrix $C_{12}\left(t, t^{\prime}\right)$, can now be written in terms of the one-particle distribution function and the initial correlation matrix $\chi_{12}\left(t_{0}\right)$. The most important feature of the short-time kinetic equation is the nonMarkovian structure of the memory functions. Let us write, for instance, the explicit expression for the memory function (7.23) using Eq. (7.39). We have

$$
W_{p_{1} p_{2}, p_{1}^{\prime} p_{2}^{\prime}}^{(B)}\left(t, t^{\prime}\right)=\left|\left\langle p_{1} p_{2}\left|\widetilde{V}_{12}\right| p_{1}^{\prime} p_{2}^{\prime}\right\rangle\right|^{2} \mathrm{e}^{-\Gamma_{p_{1} p_{2} p_{1}^{\prime} p_{2}^{\prime}\left(t, t^{\prime}\right)}} \cos \left[\Delta \omega_{p_{1} p_{2}, p_{1}^{\prime} p_{2}^{\prime}}\left(t, t^{\prime}\right)\right],
$$

where the following designation is used:

$$
\Gamma_{p_{1} p_{2} p_{1}^{\prime} p_{2}^{\prime}}\left(t, t^{\prime}\right)=\Gamma_{p_{1}}\left(t, t^{\prime}\right)+\Gamma_{p_{2}}\left(t, t^{\prime}\right)+\Gamma_{p_{1}^{\prime}}\left(t, t^{\prime}\right)+\Gamma_{p_{2}^{\prime}}\left(t, t^{\prime}\right)
$$


The structure of the memory function (7.42) is typical for the memory functions used in the delayed quantum kinetics (see, e.g., [5]), but the damping function $\Gamma_{p}\left(t, t^{\prime}\right)$ is commonly taken in a simplified form $\Gamma_{p}\left(t, t^{\prime}\right)=\gamma_{p}\left(t-t^{\prime}\right)$ with a constant $\gamma_{p}$. In some sense this corresponds to the asymptotic form of our result (7.37). It should be noted, however, that at the first stage of evolution such an approximation seems to be very rough; we see from Eq. (7.37) that the behavior of $\Gamma_{p}\left(t, t^{\prime}\right)$ is rather complicated and involves the contribution from initial correlations. Another source of memory effects is the oscillating cosine term in Eq. (7.42), which replaces the energy-conserving delta function in Boltzmann-like kinetic equations. We see that to take into account the full structure of the memory functions, even in performing the numerical evaluation of short-time quantum kinetic equations, is very complicated. Nevertheless, there are some necessary conditions which must be checked for any kinetic equation. The most important issue is whether or not the kinetic equation obeys the conservation laws. The problem of conservation laws in the delayed quantum kinetics is not trivial because of strong memory effects and the influence of initial correlations. We shall discuss the conservation laws for the kinetic equation, where the collision integrals are given by Eqs. (7.21) and (7.22). Since there are no problems with the conservation of the total number of particles and the total momentum, even for the general form of $g^{R}$ and $g^{A}$, we restrict our discussion to the conservation of energy, which is a serious problem in kinetic theory.

Since we are working in the Heisenberg picture, the average energy of the system is written as $\mathcal{E}(t)=\langle\hat{H}(t)\rangle$. In our case the Hamiltonian has the form (2.1). Going over to momentum representation and taking into account that the system is assumed to be spatially homogeneous, we find that

$$
\mathcal{E}(t)=\sum_{p_{1}} \varepsilon_{p_{1}} f_{p_{1}}(t)+\frac{1}{2} \sum_{p_{1} p_{2}}\left\langle p_{1} p_{2}\left|V_{12} \varrho_{12}(t)\right| p_{1} p_{2}\right\rangle,
$$

where $\varrho_{12}(t)$ is the nonequilibrium two-particle density matrix with the elements given by Eq. (7.15). It is convenient to separate the correlated and non-correlated parts of $\varrho_{12}(t)$ by using Eq. (7.14). Then the average energy (7.44) takes the form

$$
\mathcal{E}(t)=\mathcal{E}_{\text {kin }}(t)+\mathcal{E}_{\mathrm{HF}}(t)+\mathcal{E}_{\text {corr }}(t) .
$$

The quantity $\mathcal{E}_{\text {kin }}(t)$ is the average kinetic energy; this is just the first term in Eq. (7.44). The Hartree-Fock contribution to the average energy, $\mathcal{E}_{\mathrm{HF}}(t)$, is given by

$$
\mathcal{E}_{\mathrm{HF}}(t)=\frac{1}{2} \sum_{p_{1} p_{2}}\left\langle p_{1} p_{2}\left|\widetilde{V}_{12}\right| p_{1} p_{2}\right\rangle f_{p_{1}}(t) f_{p_{2}}(t)
$$

Finally, the term

$$
\mathcal{E}_{\text {corr }}(t)=\frac{1}{2} \sum_{p_{1} p_{2}}\left\langle p_{1} p_{2}\left|V_{12} \chi_{12}(t)\right| p_{1} p_{2}\right\rangle
$$

may be called the correlation energy. Energy conservation implies that the time derivative of $\mathcal{E}(t)$ is zero. Having a kinetic equation for $f_{p_{1}}(t)$, one can calculate

$$
\frac{d}{d t}\left(\mathcal{E}_{\mathrm{kin}}(t)+\mathcal{E}_{\mathrm{HF}}(t)\right)=\sum_{p_{1}} E_{p_{1}}(t) \frac{\partial f_{p_{1}}(t)}{\partial t}=\sum_{p_{1}} E_{p_{1}}(t) I_{p_{1}}(t),
$$


where $E_{p_{1}}(t)$ is the re-normalized particle energy, Eq. (7.32), and $I_{p_{1}}(t)$ is the collision integral. Note that the above relation is exact. Thus, the necessary condition that a kinetic equation is consistent with the energy conservation is that the sum on the righthand side of Eq. (7.48) can be represented as the time derivative. If this condition is fulfilled, then we have

$$
\sum_{p_{1}} E_{p_{1}}(t) I_{p_{1}}(t)=-\frac{d \mathcal{E}_{\mathrm{corr}}(t)}{d t} .
$$

This formula allows one to evaluate the approximate correlation energy from the kinetic equation.

It is interesting to check the condition (7.49) for the short-time kinetic equation, in which the collision integral $I_{p_{1}}(t)$ is the sum of the collision integrals $I_{p_{1}}^{(B)}(t)$ and $I_{p_{1}}^{(C)}(t)$ given by Eqs. (7.21) and (7.22). The crucial point is the choice of the retarded and advanced Green's functions entering into these collision integrals. It turns out that the necessary condition for the energy conservation is satisfied if $g_{p_{1}}^{R}\left(t, t^{\prime}\right)$ and $g_{p_{1}}^{A}\left(t^{\prime}, t\right)$ are taken in the form (7.39), where the damping function $\Gamma_{p_{1}}\left(t, t^{\prime}\right)$ is set to be equal to zero. The corresponding manipulations are straightforward but somewhat lengthy, so that we only explain the main points. Multiplying Eqs. (7.21) and (7.22) by $E_{p_{1}}(t)$ and then summing over $p_{1}$, the products of the type $E_{p}(t) g_{p}^{R}\left(t, t^{\prime}\right)$ or $g_{p}^{A}\left(t^{\prime}, t\right) E_{p}(t)$ appear in each term. These products are eliminated with the aid of Eq. (7.40) and the corresponding equation for $g_{p}^{A}\left(t^{\prime}, t\right)$ (recall that $\Gamma_{p}\left(t, t^{\prime}\right)=0$ ). It can then be shown that the $\delta$-functions do not contribute to the sum $\sum_{p} E_{p}(t) I_{p}(t)$. The remaining terms form the time derivatives of the contributions to the correlation energy. In this way, we arrive at Eq. (7.49) with the correlation energy

$$
\begin{aligned}
\mathcal{E}_{\text {corr }}(t) & =\frac{1}{4} \sum_{p_{1} p_{2} p_{1}^{\prime} p_{2}^{\prime}} \int_{t_{0}}^{t} d t^{\prime}\left|\left\langle p_{1} p_{2}\left|\widetilde{V}_{12}\right| p_{1}^{\prime} p_{2}^{\prime}\right\rangle\right|^{2} \sin \left[\Delta \omega_{p_{1} p_{2}, p_{1}^{\prime} p_{2}^{\prime}}\left(t, t^{\prime}\right)\right] \\
& \times\left(f_{p_{1}} f_{p_{2}} \bar{f}_{p_{1}^{\prime}} \bar{f}_{p_{2}^{\prime}}-\bar{f}_{p_{1}} \bar{f}_{p_{2}} f_{p_{1}^{\prime}} f_{p_{2}^{\prime}}\right)_{t^{\prime}} \\
+ & \frac{1}{2} \sum_{p_{1} p_{2}} \operatorname{Re}\left\{\left\langle p_{1} p_{2}\left|V_{12} C_{12}(t, t)\right| p_{1} p_{2}\right\rangle\right\} \\
& -\sum_{p_{1} p_{2} p_{1}^{\prime} p_{2}^{\prime}} \int_{t_{0}}^{t} d t^{\prime} \operatorname{Im}\left\{X_{p_{1} p_{2}, p_{1}^{\prime} p_{2}^{\prime}}^{(C)}\left(t, t^{\prime}\right)\right\}\left(f_{p_{1}^{\prime}} f_{p_{2}^{\prime}}-\bar{f}_{p_{1}^{\prime}} \bar{f}_{p_{2}^{\prime}}\right)_{t^{\prime}} .
\end{aligned}
$$

Here the retarded and advanced Green's functions entering into $C_{12}(t, t)$ and $X^{(C)}\left(t, t^{\prime}\right)$ are understood to be taken in the approximation described above (with $\Gamma_{p}\left(t, t^{\prime}\right)=0$ ). An attractive feature of the expression (7.50) is that it gives the exact result for the initial correlation energy. To show this, we recall Eq. (7.13), from which it follows that $C_{12}\left(t_{0}, t_{0}\right)=\chi_{12}\left(t_{0}\right)$. Thus we see that, indeed, the initial values $\mathcal{E}_{\text {corr }}\left(t_{0}\right)$ given by Eqs. (7.47) and (7.50) are identical.

Some previously obtained results follow from Eq. (7.50) as special cases. If all the contributions from initial correlations are omitted and one sets $\omega_{p}\left(t, t^{\prime}\right)=\varepsilon_{p}\left(t-t^{\prime}\right)$, the 
expression (7.50) reduces to the correlation energy calculated from the non-Markovian Boltzmann equation [32]. The second term in the correlation energy (linear in the interaction) agrees with the density operator result [12, 33] obtained by truncating the quantum hierarchy for the reduced density matrices. The last contribution to the correlation energy (7.50) stems from the "gain-loss" term in the collision integral (7.22).

It may appear tempting to improve the expressions for the collision integrals and the correlation energy by taking the retarded and advanced Green's functions in the form $(7.39)$ with $\Gamma_{p_{1}}\left(t, t^{\prime}\right) \neq 0$. This means that one goes over to the memory functions involving effects of the quasiparticle damping [see, e.g., Eq. (7.42)]. Such a procedure is often used (with an empirical exponential damping factor) in numerical calculations based on non-Markovian Boltzmann kinetic equations [5] and in many cases is crucial for the stability of the results. Unfortunately, the inclusion of the quasiparticle damping into the memory functions leads to collision integrals which do not conserve the total energy of the system. The origin of this difficulty is easy to see. We recall that the above proof of the energy conservation rests heavily on Eq. (7.40) for the retarded Green's function. If $\gamma_{p_{1}}\left(t, t^{\prime}\right)=0$, then the product $E_{p_{1}}(t) g_{p_{1}}^{R}\left(t, t^{\prime}\right)$ can exactly be expressed in terms of the time derivative of the retarded Green's function. For a finite damping, however, this is not the case, and the right-hand side of Eq. (7.49) involves extra terms violating the energy conservation. Clearly, the contribution of these extra terms to the energy balance equation is determined by the time behavior of the retarded Green's function. Strictly speaking, the total energy is conserved only if the product $\gamma_{p_{1}}\left(t, t^{\prime}\right) g_{p_{1}}^{R}\left(t, t^{\prime}\right)$ is equal to zero for all $t-t^{\prime}$. At the initial short-time stage of evolution $\gamma_{p_{1}}\left(t, t^{\prime}\right)$ is completely negligible, so that the total energy is conserved. The real difficulties appear in the long-time asymptotic behavior of the collision integrals, where $\gamma_{p_{1}}\left(t, t^{\prime}\right)$ is close to its stationary value. Then the decay of $g_{p_{1}}^{R}\left(t, t^{\prime}\right)$ must be sufficiently fast for the energy non-conserving terms in the collision integral to be negligible. For the electron scattering with optical phonons, Haug and Bányai [34] proposed a model retarded Green's function which vanishes faster than the slowly decreasing function with the constant quasiparticle damping. Their numerical results show that the collision integral with the improved $g_{p}^{R}\left(t, t^{\prime}\right)$ conserves the total energy much better in comparison to the non-Markovian collision integral with the exponential decay of the memory function. It is more natural, however, to describe the transition from the short-time dynamics to the asymptotic Boltzmann regime by using approximate self-consistent solutions of the equation for the retarded Green's function. For instance, the result (7.41) shows that the effective quasiparticle damping has a non-Markovian structure. Furthermore, it depends on the nonequilibrium one-particle distribution functions and involves contributions from initial correlations. Nevertheless, even if one uses the self-consistent expression for $g_{p}^{R}\left(t, t^{\prime}\right)$, the problem of energy conservation for all time intervals still remains open since any quasiparticle damping function $\gamma_{p}\left(t, t^{\prime}\right)$ (except for $\gamma_{p}\left(t, t^{\prime}\right)=0$ ) leads to the energy non-conserving collision integral. This is not surprising, because the extra terms in the energy balance equation are formally beyond the Born approximation used in the derivation of the kinetic equation. Thus, to obtain the collision integrals which involve the quasiparticle damping and are consistent with the energy conservation, one has to calculate the $T$-matrices in the full collision 
operators (7.7) and (7.8) keeping terms of higher-order in the interaction.

Concluding this section, we would like to touch upon another important aspect of the short-time kinetics. Suppose that the evolution of the system starts from the equilibrium state. Then a kinetic equation must lead to the obvious result $\partial f_{p}(t) / \partial t$ for all $t>t_{0}$. In other words, the collision integral must be equal to zero in thermal equilibrium. It is well known that the stationary solution of the Markovian Boltzmann equation corresponds to the equilibrium Fermi or Bose distribution function for quasiparticles. If, however, the collision integral takes account of initial correlation and memory effects, the question about the stationary solution of the kinetic equation becomes nontrivial. We have shown that the kinetic equation with the collision integrals (7.21) and (7.22) conserves the total energy (if $\Gamma_{p}\left(t, t^{\prime}\right)=0$ in the memory functions) and that the initial value of the correlation energy given by Eq. (7.50) coincides with the exact result. Due to this fact, it is reasonable to expect the kinetic equation to have the correct stationary solution. Nevertheless, we should show strictly that the collision integral vanishes in thermal equilibrium, which is a stronger condition than the conservation of energy. In Appendix $\mathrm{F}$ we calculate the collision integrals (7.21) and (7.22) up to terms of second order in the interaction using the equilibrium two-particle correlation matrix $\chi_{12}^{(\mathrm{eq})}$. The resulting kinetic equation has the form

$$
\begin{aligned}
\frac{\partial}{\partial t} f_{p_{1}}(t) & =-\sum_{p_{2} p_{1}^{\prime} p_{2}^{\prime}}\left|\left\langle p_{1} p_{2}\left|\widetilde{V}_{12}\right| p_{1}^{\prime} p_{2}^{\prime}\right\rangle\right|^{2} \int_{t_{0}}^{t} d t^{\prime} \cos \left[\Delta \omega_{p_{1} p_{2}, p_{1}^{\prime} p_{2}^{\prime}}\left(t, t^{\prime}\right)\right] \mathcal{F}_{p_{1} p_{2}, p_{1}^{\prime} p_{2}^{\prime}}\left(\left\{f\left(t^{\prime}\right)\right\}\right) \\
+ & \sum_{p_{2} p_{1}^{\prime} p_{2}^{\prime}}\left|\left\langle p_{1} p_{2}\left|\widetilde{V}_{12}\right| p_{1}^{\prime} p_{2}^{\prime}\right\rangle\right|^{2} \frac{\sin \left[\Delta \omega_{p_{1} p_{2}, p_{1}^{\prime} p_{2}^{\prime}}\left(t, t_{0}\right)\right]}{E_{p_{1}}+E_{p_{2}}-E_{p_{1}^{\prime}}-E_{p_{2}^{\prime}}} \mathcal{F}_{p_{1} p_{2}, p_{1}^{\prime} p_{2}^{\prime}}\left(\left\{f^{(\mathrm{eq})}\right\}\right)
\end{aligned}
$$

where $E_{p}$ is the particle energy in thermal equilibrium, including the Hartree-Fock contribution, and the function $\mathcal{F}$ is defined as

$$
\mathcal{F}_{p_{1} p_{2}, p_{1}^{\prime} p_{2}^{\prime}}(\{f\})=f_{p_{1}} f_{p_{2}} \bar{f}_{p_{1}^{\prime}} \bar{f}_{p_{2}^{\prime}}-\bar{f}_{p_{1}} \bar{f}_{p_{2}} f_{p_{1}^{\prime}} f_{p_{2}^{\prime}} .
$$

The first term on the right-hand side of Eq. (7.51) is the non-Markovian Boltzmann collision integral (7.21), where the quasiparticle damping is omitted. The second term is due to the equilibrium correlations. In this term $f_{p}^{(\text {eq })}$ is the equilibrium one-particle distribution function. Since, in equilibrium,

$$
\Delta \omega_{p_{1} p_{2}, p_{1}^{\prime} p_{2}^{\prime}}\left(t, t^{\prime}\right)=\left(E_{p_{1}}+E_{p_{2}}-E_{p_{1}^{\prime}}-E_{p_{2}^{\prime}}\right)\left(t-t^{\prime}\right)
$$

it can easily be seen that $f_{p_{1}}=f_{p_{1}}^{(\text {eq })}$ is a stationary solution of Eq. (7.51). Physically, this means that in equilibrium the changes in the one-particle distribution function caused by collisions and correlations exactly cancel each other. Lee et al. [10] were the first to demonstrate this fact for a weakly interacting low-density classical gas. The kinetic equation (7.51) shows that the same situation holds in quantum systems. It should also be noted that in the latter case the Hartree-Fock corrections to the particle energies have to be included, in order to assure the conservation of energy. 
The fact that in equilibrium the collision and correlation effects cancel each other is analogous to the fluctuation-dissipation theorem and suggests that correlations and fluctuations are closely related. The interplay between collisions and correlations becomes particularly clear if one neglects the Hartree-Fock terms in the memory functions. Then both terms on the right-hand side of Eq. (7.51) can be combined to give

$$
\begin{gathered}
\frac{\partial}{\partial t} f_{p_{1}}(t)=-\sum_{p_{2} p_{1}^{\prime} p_{2}^{\prime}}\left|\left\langle p_{1} p_{2}\left|\widetilde{V}_{12}\right| p_{1}^{\prime} p_{2}^{\prime}\right\rangle\right|^{2} \int_{t_{0}}^{t} d t^{\prime} \cos \left[\left(\varepsilon_{p_{1}}+\varepsilon_{p_{2}}-\varepsilon_{p_{1}^{\prime}}-\varepsilon_{p_{2}^{\prime}}\right)\left(t-t^{\prime}\right)\right] \\
\times\left[\mathcal{F}_{p_{1} p_{2}, p_{1}^{\prime} p_{2}^{\prime}}\left(\left\{f\left(t^{\prime}\right)\right\}\right)-\mathcal{F}_{p_{1} p_{2}, p_{1}^{\prime} p_{2}^{\prime}}\left(\left\{f^{(\mathrm{eq})}\right\}\right)\right] .
\end{gathered}
$$

Neglecting here the correlation term we recover the well-known Levinson equation [5, 35]. The advantage of Eq. (7.54) in comparison with the Levinson equation is that it has the right equilibrium solution. Although the correlation term was introduced in the equilibrium form, Eq. (7.54) can be considered as a rather simple and a reasonable kinetic equation describing short-time quantum kinetics in the presence of initial correlations.

\section{CONCLUSION}

We now summarize the main features of the outlined approach to short-time quantum kinetics:

1) The formalism is applicable to a wide range of correlated initial states described by nonequilibrium statistical thermodynamics.

2) Initial correlations and the time evolution are incorporated on the same footing through the many-component ("mixed") Green's functions. The essential point is that the introduction of the mixed Green's functions makes only minor changes in the standard real-time Green's function method and allows the use of the Dyson equation for correlated initial states. The existence of the Dyson equation for the mixed Green's function leads to some exact relations which play a crucial role in the derivation of kinetic equations. In particular, the generalized Kadanoff-Baym ansatz can be formulated for short-time processes in the presence of initial correlations.

3) The well-known approximations, originally developed within the standard real-time Green's method, say the T-matrix approximation, are conveniently extended to short-time kinetics with correlated initial states.

4) The method given in this paper is non-perturbative in external fields due to the self-consistency of the generalized ansatz for the real-time and cross correlation functions. This is important when considering a direct effect of a strong external field on collisions.

\section{ACKNOWLEDGMENTS}

The authors wish to express their appreciation to D. Kremp, Yu. A. Kukharenko, and K. Morawetz for valuable discussions. 


\section{APPENDIX A: \\ THERMODYNAMIC GREEN'S FUNCTIONS}

Here we briefly discuss some properties of the thermodynamic Green's functions in terms of which initial correlations are described. Owing to the structure of the evolution operator (2.12), these properties are in many ways similar to those of the MatsubaraGreen's functions [27]. In particular, the one-particle thermodynamic Green's function $\mathcal{G}\left(1,1^{\prime}\right) \equiv \mathcal{G}\left(r_{1} x_{1}, r_{1}^{\prime} x_{1}^{\prime}\right)$ depends on $x_{1}$ and $x_{1}^{\prime}$ through the difference $x_{1}-x_{1}^{\prime}$, as follows at once from the cyclic invariance of the trace in Eq. (2.14). Note also that $\mathcal{G}\left(1,1^{\prime}\right)$ is defined for $-1 \leq\left(x_{1}-x_{1}^{\prime}\right) \leq 1$, which is a necessary condition for convergence of the trace. Clearly this condition is satisfied if the "imaginary" Heisenberg picture (2.13) is introduced for $x_{0} \leq x \leq x_{0}+1$, where $x_{0}$ is an arbitrary parameter. Then it is easy to verify that $\mathcal{G}\left(1,1^{\prime}\right)$ has the property

$$
\left.\mathcal{G}\left(1,1^{\prime}\right)\right|_{x_{1}=x_{0}+1}=\left.\eta \mathcal{G}\left(1,1^{\prime}\right)\right|_{x_{1}=x_{0}},
$$

which is a generalization of the well-known Kubo-Martin-Schwinger boundary condition for the equilibrium Green's functions [27.

Perturbation expansions of the thermodynamic Green's functions can be constructed by introducing the interaction picture

$$
\hat{A}_{I}(x)=\mathrm{e}^{x \hat{S}^{0}} \hat{A} \mathrm{e}^{-x \hat{S}^{0}},
$$

where $x_{0} \leq x \leq x_{0}+1$, and $\hat{S}^{0}$ is the entropy operator for a non-correlated state [cf. Eq.(2.10)]. The interaction picture evolution operator is defined as

$$
\mathcal{U}_{I}\left(x, x^{\prime}\right)=\mathrm{e}^{x \hat{S}^{0}} \mathrm{e}^{-\left(x-x^{\prime}\right) \hat{S}} \mathrm{e}^{-x^{\prime} \hat{S}^{0}}=T_{x}^{c} \exp \left\{-\int_{x^{\prime}}^{x} d x^{\prime \prime} \hat{S}_{I}^{\prime}\left(x^{\prime \prime}\right)\right\} .
$$

Then, using the identity

$$
\mathrm{e}^{-\hat{S}}=\mathcal{U}_{I}\left(0, x_{0}\right) \mathrm{e}^{-\hat{S}^{0}} \mathcal{U}_{I}\left(x_{0}+1,0\right)
$$

which follows directly from the definition of $\mathcal{U}_{I}\left(x, x^{\prime}\right)$, the one-particle thermodynamic Green's function (2.14) can be written in the form

$$
\mathcal{G}\left(1,1^{\prime}\right)=-\frac{\left\langle T_{x}^{c}\left(\mathcal{U}_{I}\left(x_{0}+1, x_{0}\right) \psi_{I}(1) \psi_{I}^{\dagger}\left(1^{\prime}\right)\right)\right\rangle_{0}}{\left\langle\mathcal{U}_{I}\left(x_{0}+1, x_{0}\right)\right\rangle_{0}},
$$

where the symbol $\langle\ldots\rangle_{0}$ stands for averages calculated with the statistical operator

$$
\varrho^{0}\left(t_{0}\right)=\mathrm{e}^{-\hat{S}^{0}} / \operatorname{Tr}\left\{\mathrm{e}^{-\hat{S}^{0}}\right\}
$$

Expression (A.5) has the structure typical for a diagram technique. Since $\hat{S}^{0}$ is bilinear in the field operators, the statistical operator $\varrho^{0}$ admits Wick's decomposition and, consequently, expectation values of products of the interaction-picture field operators factorize 
into one-particle thermodynamic Green's functions. The corresponding Feynman rules depend on the specific form of the "correlation" term $\hat{S}^{\prime}$ in the entropy operator.

In the diagram language, the thermodynamic self-energy $\mathcal{K}$ is introduced as an irreducible part of $\mathcal{G}$. It can also be defined without reference to diagrams. To do this, let us consider equations of motion for the field operators in the Heisenberg picture (2.13). They read

$$
\begin{aligned}
& \frac{\partial}{\partial x_{1}} \psi_{H}(1)=-\int d 1^{\prime} \lambda_{1}\left(1,1^{\prime}\right) \psi_{H}\left(1^{\prime}\right)+\left[\hat{S}_{H}^{\prime}\left(x_{1}\right), \psi_{H}(1)\right], \\
& \frac{\partial}{\partial x_{1}} \psi_{H}^{\dagger}(1)=\int d 1^{\prime} \psi_{H}^{\dagger}\left(1^{\prime}\right) \lambda_{1}\left(1^{\prime}, 1\right)+\left[\hat{S}_{H}^{\prime}\left(x_{1}\right), \psi_{H}^{\dagger}(1)\right],
\end{aligned}
$$

where $d 1^{\prime}=d x_{1}^{\prime} d r_{1}^{\prime}$, and the function $\lambda_{1}\left(1,1^{\prime}\right)$ is defined as

$$
\lambda_{1}\left(1,1^{\prime}\right)=\lambda_{1}\left(r_{1}, r_{1}^{\prime}\right) \delta\left(x_{1}-x_{1}^{\prime}\right) .
$$

Then, differentiating the Green's function (2.14) with respect to $x_{1}$ and $x_{1}^{\prime}$, and using the above equations of motion for the field operators, we arrive at the Dyson equations (2.18) with the self-energy

$$
\begin{aligned}
\mathcal{K}\left(1,1^{\prime}\right) & =\int d 1^{\prime \prime}\left\langle T_{x}^{c}\left\{\left[\hat{S}_{H}^{\prime}\left(x_{1}\right), \psi_{H}(1)\right] \psi_{H}^{\dagger}\left(1^{\prime \prime}\right)\right\}\right\rangle \mathcal{G}^{-1}\left(1^{\prime \prime}, 1^{\prime}\right) \\
& =\int d 1^{\prime \prime} \mathcal{G}^{-1}\left(1,1^{\prime \prime}\right)\left\langle T_{x}^{c}\left\{\psi_{H}\left(1^{\prime \prime}\right)\left[\psi_{H}^{\dagger}\left(1^{\prime}\right), \hat{S}_{H}^{\prime}\left(x_{1}^{\prime}\right)\right]\right\}\right\rangle .
\end{aligned}
$$

These relations can be used to express the self-energy in terms of higher-order thermodynamic Green's functions. If the entropy operator is given by Eq. (2.10), we have (integration over repeated arguments is implied)

$$
\begin{aligned}
\mathcal{K}\left(1,1^{\prime}\right) & =\eta \mathcal{V}\left(12,1^{\prime \prime} 2^{\prime \prime}\right) \mathcal{G}^{(2)}\left(1^{\prime \prime} 2^{\prime \prime}, 1^{\prime \prime \prime} 2^{+}\right) \mathcal{G}^{-1}\left(1^{\prime \prime \prime}, 1^{\prime}\right) \\
& =\eta \mathcal{G}^{-1}\left(1,1^{\prime \prime \prime}\right) \mathcal{G}^{(2)}\left(1^{\prime \prime \prime} 2^{-}, 1^{\prime \prime} 2^{\prime \prime}\right) \mathcal{V}\left(1^{\prime \prime} 2^{\prime \prime}, 1^{\prime} 2\right)
\end{aligned}
$$

where the amplitude $\mathcal{V}$ is given by Eq. (4.32).

\section{APPENDIX B: \\ INTERACTION PICTURE ON THE EXTENDED CONTOUR}

Let the Heisenberg picture on the contour $\underline{C}$ of Fig. 2 be defined as

$$
\underline{\hat{A}}_{H}(\xi)=\underline{U}\left(\xi_{0}^{-}, \xi\right) \hat{A} \underline{U}\left(\xi, \xi_{0}^{-}\right),
$$

where the variable $\xi=(t, x)$ specifies a point on $\underline{C}$ and $\underline{U}\left(\xi_{1}, \xi_{2}\right)$ is the evolution operator (3.16). For the contour $\underline{C}$ shown in Fig. 2, the "effective Hamiltonian" $\underline{\hat{\mathcal{H}}}(\xi)$ coincides with $\hat{H}$ on the Keldysh part $C$ and with $-i \hat{S}$ on the parts $C_{x}^{\prime}$ and $C_{x}^{\prime \prime}$. 
The definition (B.1) of the Heisenberg picture on $\underline{C}$ allows us to introduce the pathordered ("mixed") Green's functions, as given by Eqs. (3.18) and (3.19). Due to the fact that the evolution over the Keldysh contour $C$ is described by the identity operator $\underline{U}\left(\xi_{0}^{+}, \xi_{0}^{-}\right)=1$, the mixed Green's functions coincide, up to a factor, with the thermodynamic Green's functions discussed in Sec. II and Appendix A, if all the arguments correspond to the parts $C_{x}^{\prime}$ and $C_{x}^{\prime \prime}$ of $\underline{C}$. On the other hand, if all the arguments of the mixed Green's function correspond to the Keldysh contour, then this function coincides with the real-time (path-ordered) Green's function. The latter property follows directly from the cyclic invariance of the trace in Eqs. (3.18) and (3.19).

To formulate perturbation theory for the mixed Green's functions defined on the contour $\underline{C}$ of Fig. 2, let us introduce the "interaction" picture through the relation

$$
\underline{\hat{A}}_{I}(\xi)=\underline{U}^{0}\left(\xi_{0}^{-}, \xi\right) \hat{A} \underline{U}^{0}\left(\xi, \xi_{0}^{-}\right) \text {. }
$$

The evolution operator is

$$
\underline{U}^{0}\left(\xi_{1}, \xi_{2}\right)=T_{\underline{C}} \exp \left\{-i \int_{\xi_{2}}^{\xi_{1}} \hat{\mathcal{H}}^{0}(\xi) d \xi\right\},
$$

where the unperturbed "effective Hamiltonian" $\underline{\hat{\mathcal{H}}}^{0}(\xi)$ coincides with $\hat{H}^{0}$ on the Keldysh contour $C$ and with $-i \hat{S}^{0}$ on the parts $C_{x}^{\prime}$ and $C_{x}^{\prime \prime}$ of $\underline{C}$. Then an operator in the Heisenberg picture (B.1) can be written as

$$
\underline{\hat{A}}_{H}(\xi)=\underline{U}_{I}\left(\xi_{0}^{-}, \xi\right) \underline{\hat{A}}_{I}(\xi) \underline{U}_{I}\left(\xi, \xi_{0}^{-}\right),
$$

where

$$
\underline{U}_{I}\left(\xi_{1}, \xi_{2}\right)=\underline{U}^{0}\left(\xi_{0}^{-}, \xi_{1}\right) \underline{U}\left(\xi_{1}, \xi_{2}\right) \underline{U}^{0}\left(\xi_{2}, \xi_{0}^{-}\right)
$$

is the interaction picture evolution operator. This operator is represented by a pathordered exponent

$$
\underline{U}_{I}\left(\xi_{1}, \xi_{2}\right)=T_{\underline{C}} \exp \left\{-i \int_{\xi_{2}}^{\xi_{1}} \underline{\hat{\mathcal{H}}}_{I}^{\prime}(\xi) d \xi\right\}
$$

with the effective "interaction Hamiltonian"

$$
\hat{\mathcal{H}}_{I}^{\prime}(\xi)= \begin{cases}\hat{H}_{I}^{\prime}(t) & \xi \in C, \\ -i \hat{S}_{I}^{\prime}(x) & \xi \in C_{x}^{\prime}, C_{x}^{\prime \prime} .\end{cases}
$$

Here $\hat{H}_{I}^{\prime}(t)$ is an operator in the real-time interaction picture and $\hat{S}_{I}^{\prime}(x)$ is the correlation part of the entropy operator in the thermodynamic interaction picture introduced in Appendix A. The representation (B.6) can be derived by solving the equation

$$
i \frac{\partial}{\partial \xi_{1}} \underline{U}_{I}\left(\xi_{1}, \xi_{2}\right)=\underline{\hat{\mathcal{H}}}_{I}^{\prime}\left(\xi_{1}\right) \underline{U}_{I}\left(\xi_{1}, \xi_{2}\right),
$$

which follows from Eq. (B.5). 
Let us now consider the product of Heisenberg operators $\underline{\hat{A}}_{1 H}\left(\xi_{1}\right) \cdots \underline{\hat{A}}_{k H}\left(\xi_{k}\right)$, where the points $\xi_{i}$ are arranged in a certain order on the contour $\underline{C}$ of Fig. 2. Making use of Eq. (B.4), we can write this product in terms of the interaction picture operators:

$$
\begin{aligned}
\underline{\hat{A}}_{1 H}\left(\xi_{1}\right) & \cdots \underline{\hat{A}}_{k H}\left(\xi_{k}\right) \\
& =\underline{U}_{I}\left(\xi_{0}^{-}, \xi_{1}\right) \underline{\hat{A}}_{1 I}\left(\xi_{1}\right) \underline{U}_{I}\left(\xi_{1}, \xi_{2}\right) \cdots \underline{U}_{I}\left(\xi_{k-1}, \xi_{k}\right) \underline{\hat{A}}_{k I}\left(\xi_{k}\right) \underline{U}_{I}\left(\xi_{k}, \xi_{0}^{-}\right) .
\end{aligned}
$$

As a next step to perturbation expansions of the mixed Green's functions, we use the relation

$$
\mathrm{e}^{-\hat{S}}=\underline{U}_{I}\left(\xi_{0}^{-}, \xi_{\text {in }}\right) \mathrm{e}^{-\hat{S}^{0}} \underline{U}_{I}\left(\xi_{\text {end }}, \xi_{0}^{-}\right),
$$

which is nothing but the identity (A.4) since

$$
\underline{U}_{I}\left(\xi_{0}^{-}, \xi_{\text {in }}\right)=\mathcal{U}_{I}\left(0, x_{0}\right), \quad \underline{U}_{I}\left(\xi_{\text {end }}, \xi_{0}^{-}\right)=\mathcal{U}_{I}\left(x_{0}+1,0\right),
$$

as is evident from Fig. 2. Now, combining Eqs. (B.9) and (B.10), the one-particle mixed Green's function (3.18) takes the form

$$
\underline{G}\left(1,1^{\prime}\right)=-i \frac{\left\langle T_{\underline{C}}\left\{\exp \left[-i \int_{\underline{C}} d \xi \underline{\hat{\mathcal{H}}}_{I}^{\prime}(\xi)\right] \underline{\psi}_{H}(1) \underline{\psi}_{H}^{\dagger}\left(1^{\prime}\right)\right\}\right\rangle_{0}}{\left\langle T_{\underline{C}} \exp \left[-i \int_{\underline{C}} d \xi \underline{\hat{\mathcal{H}}}_{I}^{\prime}(\xi)\right]\right\rangle_{0}}
$$

where averages are calculated over the non-correlated initial ensemble described by the statistical operator (A.6).

Note that the representation (B.12) is valid for any value of the parameter $x_{0}$ in the interval $-1 \leq x_{0} \leq 0$. It is convenient, however, to take $x_{0}=-1$ or $x_{0}=0$. To illustrate this point, let us take $x_{0}=0$ (see Fig. 3), as in the main body of the paper. Then, expanding Eq. (B.12) in terms of $\underline{\hat{\mathcal{H}}}_{I}^{\prime}(\xi)$ and applying Wick's decomposition to each term in this expansion, one will only obtain the cross Green's functions (3.21) and (3.22), in which the $x$-arguments are always later on $\underline{C}$ than the $t$-arguments. In particular, the expansion of the real-time component of $\underline{G}$ can be derived from formula

$$
G\left(1,1^{\prime}\right)=-i \frac{\left\langle\mathcal{U}_{I}(1,0) T_{C}\left\{\exp \left[-i \int_{C} d t \hat{H}_{I}^{\prime}(t)\right] \psi_{H}(1) \psi_{H}^{\dagger}\left(1^{\prime}\right)\right\}\right\rangle_{0}}{\left\langle\mathcal{U}_{I}(1,0)\right\rangle_{0}}
$$

where all the operators with the imaginary evolution are arranged to the left of operators with the real-time evolution. For a more general contour $\underline{C}$ shown in Fig. 2, one has to introduce not only the cross Green's functions (3.21) and (3.22), but also two functions

$$
\begin{aligned}
& \mathcal{F}^{>}\left(1,1^{\prime}\right)=\mathcal{F}^{>}\left(r_{1} t_{1}, r_{1}^{\prime} x_{1}^{\prime}\right)=-i\left\langle\psi_{H}\left(r_{1} t_{1}\right) \psi_{H}^{\dagger}\left(r_{1}^{\prime} x_{1}^{\prime}\right)\right\rangle, \\
& \mathcal{F}^{<}\left(1,1^{\prime}\right)=\mathcal{F}^{<}\left(r_{1} x_{1}, r_{1}^{\prime} t_{1}^{\prime}\right)=-i \eta\left\langle\psi_{H}\left(r_{1}^{\prime} t_{1}^{\prime}\right) \psi_{H}^{\dagger}\left(r_{1} x_{1}\right)\right\rangle,
\end{aligned}
$$


which appear when the $x$-argument is taken on the part $C_{x}^{\prime}$ of $\underline{C}$. It should be emphasized, however, that of the four cross functions, $\mathcal{G}^{\gtrless}$ and $\mathcal{F}^{\gtrless}$, only two are independent of each other due to relations

$$
\left[\mathcal{G}^{>}\left(1,1^{\prime}\right)\right]^{*}=-\mathcal{F}^{>}\left(1^{\prime}, \overline{1}\right), \quad\left[\mathcal{G}^{<}\left(1,1^{\prime}\right)\right]^{*}=-\mathcal{F}^{>}\left(\overline{1}^{\prime}, 1\right)
$$

where $(\bar{k})=\left(r_{k},-x_{k}\right)$.

\section{APPENDIX C: \\ An Alternative Form of Generalized KADANOFF-BAYM EQUATIONS}

We shall discuss the connection between our equations (4.17), (4.19), and those derived by the diagram technique [18]. First of all we note that Eqs. (5.8) yield the following expressions for the correlation terms appearing in the generalized Kadanoff-Baym equations:

$$
\mathcal{K}^{<} \mathcal{G}^{>}=i \mathcal{K}^{<} \mathcal{G} \mathcal{K}^{>} g^{A}+\mathcal{K}^{<} \mathcal{G}_{\text {hom }}^{>}, \quad \mathcal{G}^{<} \mathcal{K}^{>}=i g^{R} \mathcal{K}^{<} \mathcal{G} \mathcal{K}^{>}+\mathcal{G}_{\text {hom }}^{<} \mathcal{K}^{>}
$$

We now make use of Eqs. (5.11) to write

$$
\mathcal{G}_{\text {hom }}^{<} \mathcal{K}^{>}=g^{R} \Sigma^{C}, \quad \mathcal{K}^{<} \mathcal{G}_{\text {hom }}^{>}=\Sigma_{C} g^{A},
$$

where we have introduced the singular self-energies

$$
\begin{aligned}
& \Sigma^{C}\left(t_{1}, t_{2}\right)=i \delta\left(t_{1}-t_{0}-0\right) \mathcal{G}^{<}\left(t_{0}\right) \mathcal{K}^{>}\left(t_{2}\right) . \\
& \Sigma_{C}\left(t_{1}, t_{2}\right)=-i \mathcal{K}^{<}\left(t_{1}\right) \mathcal{G}^{>}\left(t_{0}\right) \delta\left(t_{2}-t_{0}-0\right) .
\end{aligned}
$$

Combining Eqs. (C.1) with (C.2), we see that Eqs. (4.17) and (4.19) can be rewritten in the form

$$
\begin{aligned}
& \left(g_{0}^{-1}-\Sigma^{R}\right) g^{\gtrless}=\left(\bar{\Sigma}^{\gtrless}+\Sigma_{C}\right) g^{A}, \\
& g^{\gtrless}\left(g_{0}^{-1}-\Sigma^{A}\right)=g^{R}\left(\bar{\Sigma}^{\gtrless}+\Sigma^{C}\right),
\end{aligned}
$$

where

$$
\bar{\Sigma}^{\gtrless}=\Sigma^{\gtrless}+i \mathcal{K}<\mathcal{G} \mathcal{K}^{>}
$$

are the re-normalized real-time components of the self-energy.

Equations (C.5) and (C.6) are similar to the equations derived by Danielewicz from diagram expansions of Green's functions [36]. In the diagram language, $\bar{\Sigma}{ }^{\gtrless}$ are irreducible parts of the real-time Green's function, which begin and end with an interaction amplitude in the Hamiltonian $\hat{H}$ [18]. On the other hand, the singular self-energy $\Sigma^{C}\left(\Sigma_{C}\right)$ is 
represented by diagrams which begin (end) with a correlation matrix and end (begin) with the interaction amplitude $V$. In our approach, such a structure of the above selfenergies follows directly from equations of motion for $\mathcal{G}^{\gtrless}$, Eqs. (4.24) and (4.27), since the function $\mathcal{K}^{<}\left(\mathcal{K}^{>}\right)$contains the interaction amplitude $V$ as a left-side (right-side) multiplier.

\section{APPENDIX D: SELF-ENERGY ON THE EXTENDED CONTOUR}

If the entropy operator (2.9) contains a finite number of the correlation terms, one can derive a hierarchy of equations for the mixed Green's functions, which is analogous to the Martin-Schwinger hierarchy in the standard real-time Green's function formalism [28]. Truncation of this new hierarchy at some order allows one to formulate reasonable approximations for the self-energy on the extended contour $\underline{C}$, as much as truncation of the Martin-Schwinger hierarchy is used for evaluation of the self-energy on the Keldysh contour $C$. Here we will discuss briefly the connection between the self-energy $\Sigma$ and higher-order mixed Green's functions in the model where the entropy operator includes only the two-particle correlation term, Eq. (2.10).

The hierarchy of equations for the mixed Green's functions follows directly from equations of motion for the field operators $\underline{\psi}_{H}(1)$ and $\underline{\psi}_{H}^{\dagger}(1)$ in the Heisenberg picture on the extended contour $\underline{C}$ :

$$
i \frac{\partial}{\partial \xi_{1}} \underline{\psi}_{H}(1)=\left[\underline{\psi}_{H}(1), \underline{\hat{\mathcal{H}}}\left(\xi_{1}\right)\right], \quad i \frac{\partial}{\partial \xi_{1}} \underline{\psi}_{H}^{\dagger}(1)=\left[\underline{\psi}_{H}^{\dagger}(1), \underline{\hat{\mathcal{H}}}\left(\xi_{1}\right)\right] .
$$

Recalling the definition (3.17) of the "effective Hamiltonian" on the contour $\underline{C}$, the above equations can be transformed to (with integration over repeated arguments)

$$
\begin{aligned}
& \underline{G}_{0}^{-1}\left(1,1^{\prime}\right) \underline{\psi}_{H}\left(1^{\prime}\right)=\underline{V}\left(12,1^{\prime} 2^{\prime}\right) \underline{\psi}_{H}^{\dagger}(2) \underline{\psi}_{H}\left(2^{\prime}\right) \underline{\psi}_{H}\left(1^{\prime}\right), \\
& \underline{\psi}_{H}^{\dagger}\left(1^{\prime}\right) \underline{G}_{0}^{-1}\left(1^{\prime}, 1\right)=\underline{V}\left(1^{\prime} 2^{\prime}, 12\right) \underline{\psi}_{H}^{\dagger}\left(1^{\prime}\right) \underline{\psi}_{H}^{\dagger}\left(2^{\prime}\right) \underline{\psi}_{H}(2),
\end{aligned}
$$

where the operator $\underline{G}_{0}^{-1}$ and the "interaction amplitude" $\underline{V}$ on the contour $\underline{C}$ are defined by Eq. (4.3) and Eq. (4.30), respectively. Now $\underline{G}_{0}^{-1}$ operating on the Green's function (3.18) from the left and right and then using Eqs. (D.2) results in the following equations:

$$
\begin{aligned}
& \underline{G}_{0}^{-1}\left(1,1^{\prime \prime}\right) \underline{G}\left(1^{\prime \prime}, 1^{\prime}\right)=\underline{\delta}\left(1,1^{\prime}\right)+i \eta \underline{V}\left(12,1^{\prime \prime} 2^{\prime \prime}\right) \underline{G}^{(2)}\left(1^{\prime \prime} 2^{\prime \prime}, 1^{\prime} 2^{+}\right), \\
& \underline{G}\left(1,1^{\prime \prime}\right) \underline{G}_{0}^{-1}\left(1^{\prime \prime}, 1^{\prime}\right)=\underline{\delta}\left(1,1^{\prime}\right)+i \eta \underline{G}^{(2)}\left(12^{-}, 1^{\prime \prime} 2^{\prime \prime}\right) \underline{V}\left(1^{\prime \prime} 2^{\prime \prime}, 1^{\prime} 2\right) .
\end{aligned}
$$

These are the first equations of the hierarchy for the mixed Green's functions on the contour $\underline{C}$, which is the analogue of the Martin-Schwinger hierarchy in the standard realtime Green's function formalism [28]. Assuming that $\underline{G}$ has an inverse on the contour $\underline{C}$, 
Eqs. (D.3) can be written as Dyson equations (4.1) and (4.2) with the matrix self-energy $\underline{\Sigma}$ given by Eq. (4.29).

We emphasize that it has been possible to express the self-energy $\underline{\Sigma}$ in terms of the twoparticle mixed Green's function $\underline{G}^{(2)}$ due to the structure of the entropy operator (2.10). For a more general form of the entropy operator, the self-energy $\underline{\Sigma}$ will depend on higherorder mixed Green's functions.

\section{APPENDIX E: \\ The Two-Particle Time CORRELATiOn Matrix}

To calculate the time correlation matrix (7.9), we use the ansatz (7.11) for the cross Green's functions and find that

$$
C_{12}\left(t, t^{\prime}\right)=g_{12}^{R}\left(t, t_{0}\right) \mathcal{G}_{12}^{<}\left(t_{0}\right) \widetilde{\mathcal{T}}_{12} \mathcal{G}_{12}^{>}\left(t_{0}\right) g_{12}^{A}\left(t_{0}, t^{\prime}\right) .
$$

Let us now turn to Eqs. (6.13) for the thermodynamic T-matrix. Recalling Eq. (6.22) and the boundary conditions (3.23), we write

$$
\mathcal{G}_{12}^{<}\left(t_{0}\right) \widetilde{\mathcal{T}}_{12} \mathcal{G}_{12}^{>}\left(t_{0}\right)=\int_{0}^{1} d x d x^{\prime} \mathcal{G}_{12}(0, x) \widetilde{\mathcal{T}}_{12}\left(x, x^{\prime}\right) \mathcal{G}_{12}\left(x^{\prime}, 0\right),
$$

where we have introduced the notation

$$
\mathcal{G}_{12}\left(x, x^{\prime}\right)=\mathcal{G}_{1}\left(x, x^{\prime}\right) \mathcal{G}_{2}\left(x, x^{\prime}\right) .
$$

We now wish to show that the right-hand side of Eq. (E.2) can be expressed in terms of the two-particle thermodynamic Green's function $\mathcal{G}^{(2)}$ [see Eq. (2.15)]. To that end we use Eq. (6.7) which, when written for the thermodynamic component of $\underline{G}^{(2)}$, reads

$$
\begin{aligned}
\mathcal{G}^{(2)}\left(12,1^{\prime} 2^{\prime}\right)= & \left(\mathcal{G}\left(1,1^{\prime}\right) \mathcal{G}\left(2,2^{\prime}\right)\right)_{\mathrm{ex}} \\
& +\mathcal{G}\left(1,1^{\prime \prime}\right) \mathcal{G}\left(2,2^{\prime \prime}\right) \widetilde{\mathcal{T}}\left(1^{\prime \prime} 2^{\prime \prime}, 1^{\prime \prime \prime} 2^{\prime \prime \prime}\right) \mathcal{G}\left(1^{\prime \prime \prime}, 1^{\prime}\right) \mathcal{G}\left(2^{\prime \prime \prime}, 2^{\prime}\right)
\end{aligned}
$$

It is convenient here to go to the matrix notation with respect to the single-particle quantum numbers. Defining the matrix $\mathcal{G}_{12}^{(2)}\left(x_{1} x_{2}, x_{1}^{\prime} x_{2}^{\prime}\right)$ by

$$
\mathcal{G}^{(2)}\left(12,1^{\prime} 2^{\prime}\right)=\left\langle r_{1} r_{2}\left|\mathcal{G}_{12}^{(2)}\left(x_{1} x_{2}, x_{1}^{\prime} x_{2}^{\prime}\right)\right| r_{1}^{\prime} r_{2}^{\prime}\right\rangle,
$$

we have

$$
\begin{aligned}
& \mathcal{G}_{12}^{(2)}\left(x_{1} x_{2}, x_{1}^{\prime} x_{2}^{\prime}\right)=\left(\mathcal{G}_{1}\left(x_{1}, x_{1}^{\prime}\right) \mathcal{G}_{2}\left(x_{2}, x_{2}^{\prime}\right)\right)_{\mathrm{ex}} \\
& \quad+\int_{0}^{1} d x^{\prime \prime} d x^{\prime \prime \prime} \mathcal{G}_{1}\left(x_{1}, x^{\prime \prime}\right) \mathcal{G}_{2}\left(x_{2}, x^{\prime \prime}\right) \widetilde{\mathcal{T}}_{12}\left(x^{\prime \prime}, x^{\prime \prime \prime}\right) \mathcal{G}_{1}\left(x^{\prime \prime \prime}, x_{1}^{\prime}\right) \mathcal{G}_{2}\left(x^{\prime \prime \prime}, x_{2}^{\prime}\right)
\end{aligned}
$$


A comparison with Eq. (E.2) shows that

$$
\mathcal{G}_{12}^{<}\left(t_{0}\right) \widetilde{\mathcal{T}}_{12} \mathcal{G}_{12}^{>}\left(t_{0}\right)=\lim _{\substack{x \rightarrow 0 \\ x^{\prime} \rightarrow 0}}\left\{\mathcal{G}_{12}^{(2)}\left(x x, x^{\prime} x^{\prime}\right)-\left(\mathcal{G}_{1}\left(x, x^{\prime}\right) \mathcal{G}_{2}\left(x, x^{\prime}\right)\right)_{\mathrm{ex}}\right\}
$$

Using the obvious relation

$$
\begin{gathered}
\left\langle r_{1} r_{2}\left|\mathcal{G}_{12}^{(2)}\left(x x, x^{\prime} x^{\prime}\right)\right| r_{1}^{\prime} r_{2}^{\prime}\right\rangle=\theta\left(x-x^{\prime}\right)\left\langle\psi_{H}\left(r_{1} x\right) \psi_{H}\left(r_{2} x\right) \psi_{H}^{\dagger}\left(r_{2}^{\prime} x^{\prime}\right) \psi_{H}^{\dagger}\left(r_{1}^{\prime} x^{\prime}\right)\right\rangle \\
+\theta\left(x^{\prime}-x\right)\left\langle\psi_{H}^{\dagger}\left(r_{2}^{\prime} x^{\prime}\right) \psi_{H}^{\dagger}\left(r_{1}^{\prime} x^{\prime}\right) \psi_{H}\left(r_{1} x\right) \psi_{H}\left(r_{2} x\right)\right\rangle
\end{gathered}
$$

it is easy to verify that the order of the limits in Eq. (E.7) is of no significance, as it should be. In both cases we obtain the same result

$$
\mathcal{G}_{12}^{<}\left(t_{0}\right) \widetilde{\mathcal{T}}_{12} \mathcal{G}_{12}^{>}\left(t_{0}\right)=\chi_{12}\left(t_{0}\right)
$$

where $\chi_{12}\left(t_{0}\right)$ is the initial two-particle correlation matrix (7.14). Relations (E.1) and (E.9) complete the derivation of Eq. (7.13).

\section{APPENDIX F: Derivation of the Kinetic Equation With Equilibrium CORRELATIONS}

The first (Boltzmann) term on the right-hand side of Eq. (7.51) follows immediately from Eq. (7.21) if we take the memory function $W^{(B)}$ in the form $(7.42)$ with $\Gamma_{p}=0$. Thus it remains to evaluate the correlation term given by Eq. (7.22). Since in the case of equilibrium correlations the matrix $C_{12}\left(t, t^{\prime}\right)$ is proportional to $V_{12}$ and we wish to obtain $I^{(C)}$ to order $V_{12}^{2}$, we can restrict our discussion to the first term in Eq. (7.22). We start by evaluating the equilibrium correlation matrix

$$
\left\langle p_{1} p_{2}\left|\chi_{12}^{(\mathrm{eq})}\right| p_{1}^{\prime} p_{2}^{\prime}\right\rangle=\left\langle a_{p_{2}^{\prime}}^{\dagger} a_{p_{1}^{\prime}}^{\dagger} a_{p_{1}} a_{p_{2}}\right\rangle_{\mathrm{eq}}-f_{p_{1}}^{(\mathrm{eq})} f_{p_{2}}^{(\mathrm{eq})}\left(\delta_{p_{1} p_{1}^{\prime}} \delta_{p_{2} p_{2}^{\prime}}+\eta \delta_{p_{1} p_{2}^{\prime}} \delta_{p_{2} p_{1}^{\prime}}\right)
$$

where the symbol $\langle\cdots\rangle_{\text {eq }}$ stands for averages calculated with the statistical operator (2.5), and $f_{p}^{(\text {eq })}=\left\langle a_{p}^{\dagger} a_{p}\right\rangle_{\text {eq }}$ is the equilibrium one-particle distribution function. The secondquantized Hamiltonian in momentum representation is given by

$$
\hat{H}=\hat{H}^{0}+\hat{H}^{\prime}=\sum_{p_{1}} \varepsilon_{p_{1}} a_{p_{1}}^{\dagger} a_{p_{1}}+\frac{1}{2} \sum_{p_{1} p_{2} p_{1}^{\prime} p_{2}^{\prime}}\left\langle p_{1} p_{2}\left|V_{12}\right| p_{1}^{\prime} p_{2}^{\prime}\right\rangle a_{p_{2}^{\prime}}^{\dagger} a_{p_{1}^{\prime}}^{\dagger} a_{p_{1}} a_{p_{2}} .
$$

Recall that we need to evaluate the correlation matrix to first order in the interaction. This can be done by elementary methods by expanding the statistical operator (2.5) in $\hat{H}^{\prime}$. It is convenient, however, to redefine the unperturbed Hamiltonian by taking the Hartree-Fock term into the particle energies. Then, instead of Eq. (2.5) we now have

$$
\varrho_{\text {eq }}=\mathrm{e}^{-\beta\left(\hat{\mathcal{H}}^{0}+\hat{\mathcal{H}}^{\prime}\right)} / \operatorname{Tr} \mathrm{e}^{-\beta\left(\hat{\mathcal{H}}^{0}+\hat{\mathcal{H}}^{\prime}\right)},
$$


where we have defined

$$
\begin{aligned}
\hat{\mathcal{H}}^{0} & =\sum_{p_{1}}\left(E_{p_{1}}-\mu\right) a_{p_{1}}^{\dagger} a_{p_{1}}, \\
\hat{\mathcal{H}}^{\prime} & =\frac{1}{4} \sum_{p_{1} p_{2} p_{1}^{\prime} p_{2}^{\prime}}\left\langle p_{1} p_{2}\left|\widetilde{V}_{12}\right| p_{1}^{\prime} p_{2}^{\prime}\right\rangle a_{p_{2}^{\prime}}^{\dagger} a_{p_{1}^{\prime}}^{\dagger} a_{p_{1}} a_{p_{2}}-\sum_{p_{1}} \Sigma_{p_{1}}^{\mathrm{HF}} a_{p_{1}}^{\dagger} a_{p_{1}} .
\end{aligned}
$$

The re-normalized particle energies are given by

$$
E_{p_{1}}=\varepsilon_{p_{1}}+\Sigma_{p_{1}}^{\mathrm{HF}}=\varepsilon_{p_{1}}+\sum_{p_{2}}\left\langle p_{1} p_{2}\left|\tilde{V}_{12}\right| p_{1} p_{2}\right\rangle f_{p_{2}}^{(\mathrm{eq})}
$$

Up to terms linear in $\mathcal{H}^{\prime}$ Eq. (F.3) reads

$$
\varrho_{\text {eq }}=\left[1-\int_{0}^{\beta} d \lambda \mathrm{e}^{-\lambda \hat{\mathcal{H}}^{0}}\left(\hat{\mathcal{H}}^{\prime}-\left\langle\hat{\mathcal{H}}^{\prime}\right\rangle_{0}\right) \mathrm{e}^{\lambda \hat{\mathcal{H}}^{0}}\right] \varrho_{\text {eq }}^{0}
$$

where the statistical operator

$$
\varrho_{\text {eq }}^{0}=\mathrm{e}^{-\beta \hat{\mathcal{H}}^{0}} / \operatorname{Tr} \mathrm{e}^{-\beta \hat{\mathcal{H}}^{0}}
$$

describes the ideal quantum gas with the quasiparticle energies (F.6). The symbol $\langle\cdots\rangle_{0}$ means the average with $\varrho_{\text {eq }}^{0}$. Making use of Eq. ( $F$ F.7 $)$, it is easy to see that to first order in the interaction the one-particle distribution can be replaced by the Fermi or Bose distribution

$$
f_{p}^{(\mathrm{eq})}=\left[\mathrm{e}^{\beta\left(E_{p}-\mu\right)}-\eta\right]^{-1}
$$

We now can evaluate the average value in Eq. (F.1) with the aid of Eq. (F.7). Using the relations

$$
\mathrm{e}^{\lambda \hat{\mathcal{H}}^{0}} a_{p} \mathrm{e}^{-\lambda \hat{\mathcal{H}}^{0}}=\mathrm{e}^{-\lambda\left(E_{p}-\mu\right)} a_{p}, \quad \mathrm{e}^{\lambda \hat{\mathcal{H}}^{0}} a_{p}^{\dagger} \mathrm{e}^{-\lambda \hat{\mathcal{H}}^{0}}=\mathrm{e}^{\lambda\left(E_{p}-\mu\right)} a_{p}^{\dagger}
$$

we find that

$$
\left\langle p_{1} p_{2}\left|\chi_{12}^{(\mathrm{eq})}\right| p_{1}^{\prime} p_{2}^{\prime}\right\rangle=\frac{1}{E_{p_{1} p_{2}}-E_{p_{1}^{\prime} p_{2}^{\prime}}}\left(\mathrm{e}^{-\beta\left(E_{p_{1} p_{2}}-E_{p_{1}^{\prime} p_{2}^{\prime}}\right)}-1\right)\left\langle a_{p_{2}^{\prime}}^{\dagger} a_{p_{1}^{\prime}}^{\dagger} a_{p_{1}} a_{p_{2}} \hat{\mathcal{H}}^{\prime}\right\rangle_{0}^{(c)},
$$

where $E_{p_{1} p_{2}}=E_{p_{1}}-E_{p_{2}}$. In the above expression the superscript $(c)$ shows that only the connected part of the average must be taken. This means that, in applying Wick's theorem, all the creation and annihilation operators in $\mathcal{H}^{\prime}$ must be contracted with the "free" operators. Further manipulations are very simple. After some algebra we find

$$
\left\langle p_{1} p_{2}\left|\chi_{12}^{(\mathrm{eq})}\right| p_{1}^{\prime} p_{2}^{\prime}\right\rangle=\frac{\mathcal{F}_{p_{1} p_{2}, p_{1}^{\prime} p_{2}^{\prime}}\left(\left\{f^{(\mathrm{eq})}\right\}\right)}{E_{p_{1} p_{2}}-E_{p_{1}^{\prime} p_{2}^{\prime}}}\left\langle p_{1} p_{2}\left|\widetilde{V}_{12}\right| p_{1}^{\prime} p_{2}^{\prime}\right\rangle
$$

where the function $\mathcal{F}(\{f\})$ is defined by Eq. (7.52). In writing Eq. (F.12), the temperature dependent factors have been eliminated by means of Eq. (F.9).

Now we substitute the expression (F.12) into Eq. (7.13) and take the retarded and advanced Green's functions in the form (7.39) with $\Gamma_{p}=0$. This gives us the timecorrelation function $C_{12}(t, t)$ which is to be used for evaluating the first term in the collision integral (7.22), i.e., the correlation contribution into the kinetic equation (7.51). 


\section{References}

[1] R. Zwanzig, J. Chem. Phys. 33 (1960), 1338.

[2] I. Prigogine and P. Résibois, Physica 27 (1961), 629.

[3] R. Balescu, Physica 38 (1968), 98.

[4] J. Shan, "Ultrafast Spectroscopy of Semiconductors and Semiconductor Microstructures", Springer Series in Solid-State Sci., Vol. 115, Springer, Berlin/Heidelberg, 1996.

[5] H. Haug and A.-P. Jauho, "Quantum Kinetics in Transport and Optics of Semiconductors", Springer Series in Solid-State Sci., Vol. 123, Springer, Berlin/Heidelberg, 1996.

[6] P. Danielewicz, Ann. Phys. (N.Y.) 55 (1984), 305.

[7] C. Greiner, K. Wagner, and P. G. Reinhard, Phys. Rev. C 49 (1994), 1693.

[8] H. S. Köhler, Phys. Rev. C 51 (1995), 3232.

[9] H.S. Köhler, Phys. Rev. E 53 (1996), 3145.

[10] D. Lee, S. Fujita, and F. Wu, Phys. Rev. A 2 (1970), 854.

[11] D. Kremp, M. Bonitz, W.D. Kraeft, and M. Schlanges, Ann. Phys. (N.Y.) 258 (1997), 320 .

[12] M. Bonitz, "Quantum Kinetic Theory", Teubner, Stuttgart/Leipzig, 1998.

[13] L. P. Kadanoff and G. Baym, "Quantum Statistical Mechanics", Benjamin, N.Y., 1962.

[14] L. V. Keldysh, Zh. Eksp. Teor. Fiz. 47 (1964), 1515 [Sov. Phys.- JETP 20 (1965), 1018].

[15] A. J. Hall, J. Phys. A 8 (1975), 214.

[16] A. J. Hall, Physica A 80 (1975), 369.

[17] Yu.A. Kukharenko and S.G. Tikhodeev, Zh. Eksp. Teor. Fiz. 83 (1982), 1444 [Sov. Phys.- JETP 56 (1982), 831].

[18] P. Danielewicz, Ann. Phys. (N.Y.) 55 (1984), 239.

[19] M. Wagner, Phys. Rev. B 44 (1991), 6104.

[20] D. Semkat, D. Kremp, and M. Bonitz, Phys. Rev. E 59 (1999), 1557. 
[21] P. Lipavský, V. Špička, and B. Veliký, Phys. Rev. B 34 (1986), 6933.

[22] E. T. Jaynes, Phys. Rev. 106 (1957), 620; 108 (1957), 171.

[23] E. T. Jaynes, "Information Theory and Statistical Mechanics". In: "Statistical Physics. Brandeis Lectures", Vol.3, Benjamin, N.Y., 1963, p. 181.

[24] D. N. Zubarev, V. G. Morozov, and G. Röpke, "Statistical Mechanics of Nonequilibrium Processes", Vol. 1, Akademie Verlag, Berlin, 1996.

[25] D. N. Zubarev, V. G. Morozov, and G. Röpke, "Statistical Mechanics of Nonequilibrium Processes", Vol. 2, Akademie Verlag, Berlin, 1997.

[26] G. Röpke, Ann. Physik (Leipzig) 3 (1994), 145.

[27] A.L. Fetter and J.D. Walecka, "Quantum Theory of Many-Particle Systems", McGraw-Hill, N.Y., 1971.

[28] W. Botermans and R. Malfliet, Phys. Rep. 198 (1990), 115.

[29] R. A. Craig, J. Math. Phys. 9 (1968), 605.

[30] D. Kremp, M. Schlanges, and Th. Bornath, J. Stat. Phys. 41 (1985), 661.

[31] K. Morawetz and G. Röpke, Phys. Rev. E 51 (1995), 4246.

[32] K. Morawetz, Phys. Lett. A 199 (1995), 241.

[33] M. Bonitz, D. Kremp, D.C. Scott, R. Binder, W.D. Kraeft, and H.S. Köhler, Journal of Physics: Condensed Matter 8 (1996) 6057.

[34] H. Haug and L. Bányai, Solid St. Commun. 100 (1996) 303.

[35] I.B. Levinson, Zh. Eksp. Teor. Fiz. 57 (1969) [ Sov. Phys. - JETP 30 (1970) 362 ].

[36] It should be noted that, in Danielewicz's paper [18], there is no clear distinction between the two different self-energies, $\Sigma^{\gtrless}$ and $\bar{\Sigma}^{\gtrless}$. In Eq. (5.22) of Ref. [18], the $\Sigma^{\gtrless}$ have the same meaning as in the present paper. On the other hand, in Eqs. (H.13) and (H.14) of Ref. [18] the symbols $\Sigma^{\gtrless}$ denote sets of diagrams which correspond to the re-normalized self-energies $\bar{\Sigma}^{\gtrless}$ as given by our Eq. (C.7). 


\section{FIGURES}

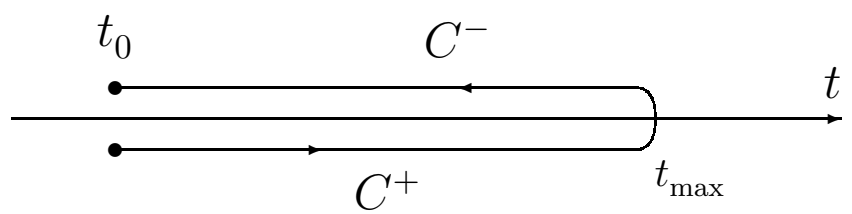

Figure 1

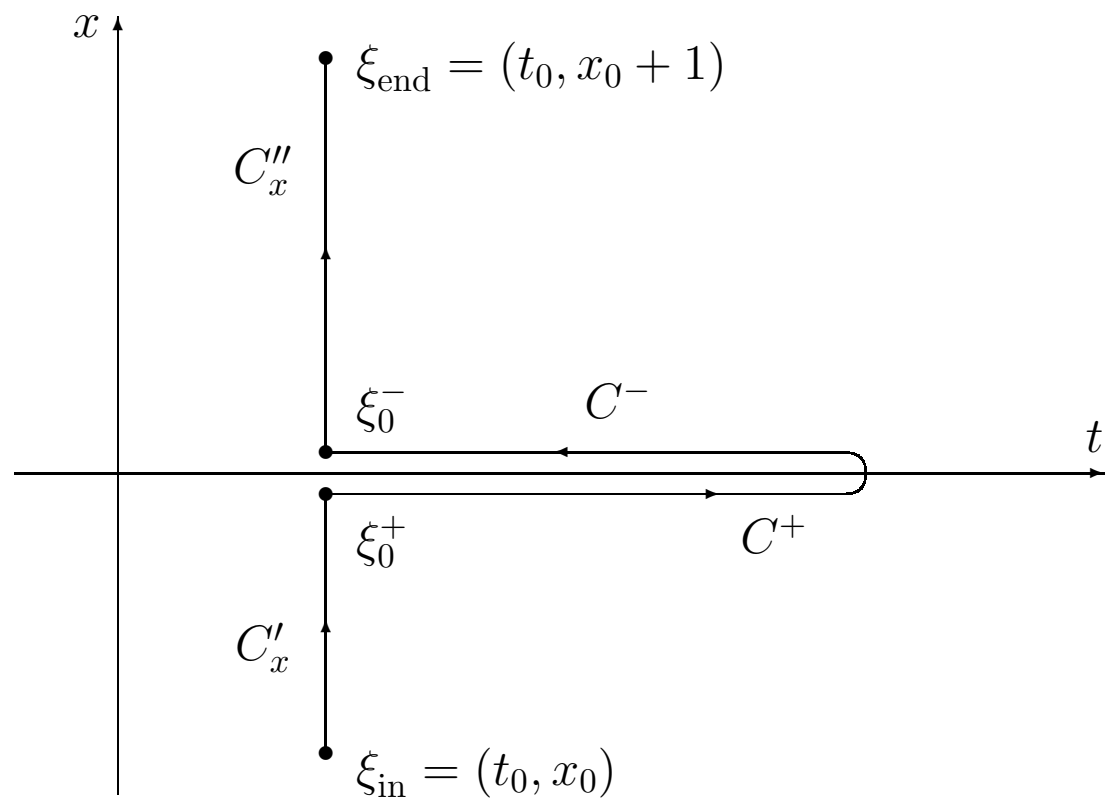

Figure 2 


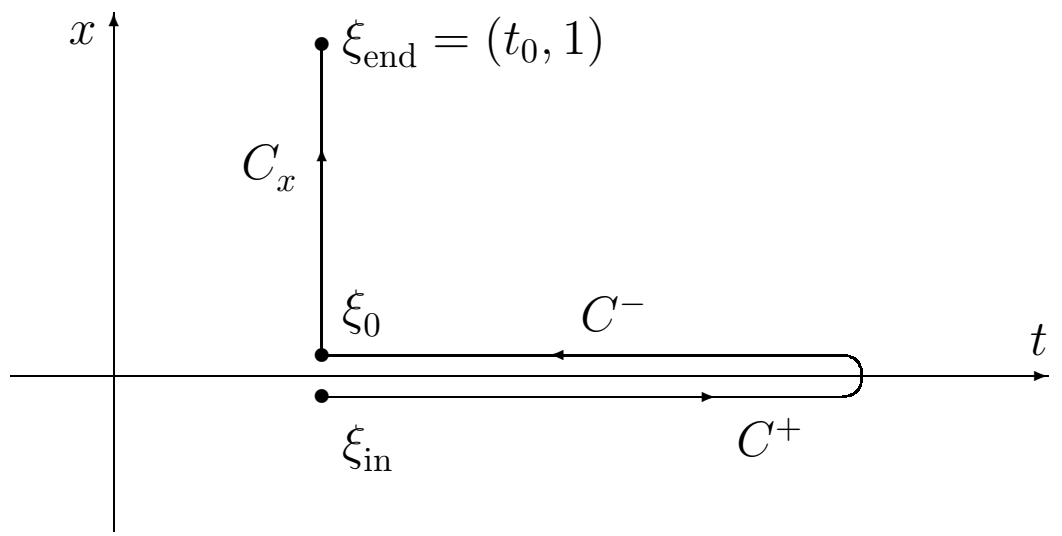

Figure 3 


\section{FIGURE CAPTIONS}

Figure 1:

The Keldysh contour $C$ with the lower (chronological) branch $C^{+}$and the upper (antichronological) branch $C^{-}$

Figure 2:

The extended contour $\underline{C}$ with the real-time evolution on the Keldysh contour $C$ and the "imaginary" evolution on the parts $C_{x}^{\prime}$ and $C_{x}^{\prime \prime}$. The parameter $x_{0}$ satisfies $-1 \leq x_{0} \leq 0$.

\section{Figure 3:}

A special case $\left(x_{0}=0\right)$ of the contour shown in Fig. 2 